\title{
Topological T-Duality for Twisted Tori
}

Paolo ASCHIERI ${\dagger^{1} \dagger^{2} \dagger^{3}}^{2}$ and Richard J. SZABO ${ }^{\dagger^{1} \dagger^{2} \dagger^{4} \dagger^{5} \dagger^{6}}$

$\dagger^{1}$ Dipartimento di Scienze e Innovazione Tecnologica, Università del Piemonte Orientale, Viale T. Michel 11, 15121 Alessandria, Italy

E-mail: paolo.aschieri@uniupo.it

$\dagger^{2}$ Arnold-Regge Centre, Via P. Giuria 1, 10125 Torino, Italy

$\dagger^{3}$ Istituto Nazionale di Fisica Nucleare, Torino, Via P. Giuria 1, 10125 Torino, Italy

$\dagger^{4}$ Department of Mathematics, Heriot-Watt University, Colin Maclaurin Building, Riccarton, Edinburgh EH14 4AS, UK

E-mail: R.J.Szabo@hw.ac.uk

$\dagger^{5}$ Maxwell Institute for Mathematical Sciences, Edinburgh, UK

$\dagger^{6}$ Higgs Centre for Theoretical Physics, Edinburgh, UK

Received June 30, 2020, in final form January 22, 2021; Published online February 05, 2021

https://doi.org/10.3842/SIGMA.2021.012

\begin{abstract}
We apply the $C^{*}$-algebraic formalism of topological T-duality due to Mathai and Rosenberg to a broad class of topological spaces that include the torus bundles appearing in string theory compactifications with duality twists, such as nilmanifolds, as well as many other examples. We develop a simple procedure in this setting for constructing the T-duals starting from a commutative $C^{*}$-algebra with an action of $\mathbb{R}^{n}$. We treat the general class of almost abelian solvmanifolds in arbitrary dimension in detail, where we provide necessary and sufficient criteria for the existence of classical T-duals in terms of purely group theoretic data, and compute them explicitly as continuous-trace algebras with non-trivial DixmierDouady classes. We prove that any such solvmanifold has a topological T-dual given by a $C^{*}$ algebra bundle of noncommutative tori, which we also compute explicitly. The monodromy of the original torus bundle becomes a Morita equivalence among the fiber algebras, so that these $C^{*}$-algebras rigorously describe the T-folds from non-geometric string theory.
\end{abstract}

Key words: noncommutative $C^{*}$-algebraic T-duality; nongeometric backgrounds; Mostow fibration of almost abelian solvmanifolds; $C^{*}$-algebra bundles of noncommutative tori

2020 Mathematics Subject Classification: 46L55; 81T30; 16D90

\section{Dedicated to Giovanni Landi on the occasion of his 60th birthday}

\section{Contents}

1 Introduction $\quad 2$

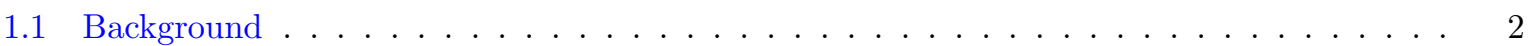

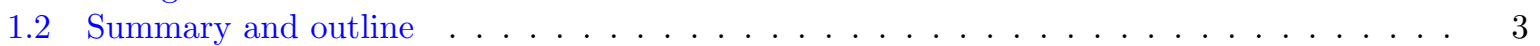

2 Crossed products and duality $\mathbf{5}$

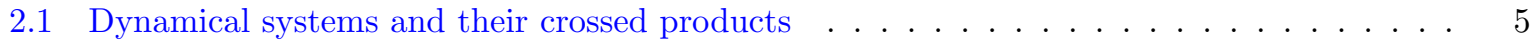

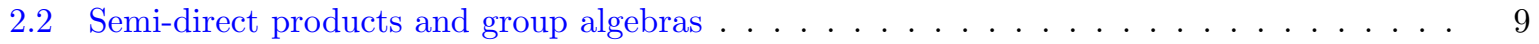

2.3 Pontryagin duality and Fourier transform . . . . . . . . . . . . . . . 11

2.4 Morita equivalence and Green's theorem . . . . . . . . . . . . . . . . . . 12

This paper is a contribution to the Special Issue on Noncommutative Manifolds and their Symmetries in honour of Giovanni Landi. The full collection is available at https://www.emis.de/journals/SIGMA/Landi.html 
3 Topological T-duality and twisted tori $\quad 16$

3.1 Twisted tori and their T-duals $\ldots \ldots \ldots \ldots \ldots \ldots$

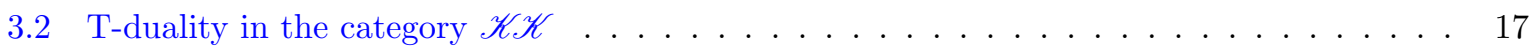

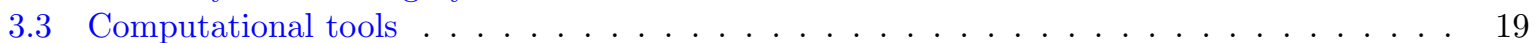

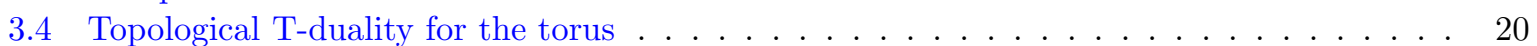

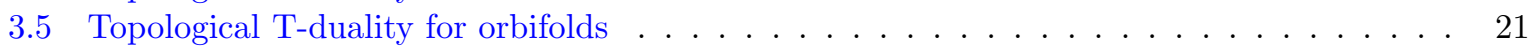

4 Topological T-duality for almost abelian solvmanifolds $\quad 22$

4.1 Mostow bundles . . . . . . . . . . . . . . . . . . . . . . . 22

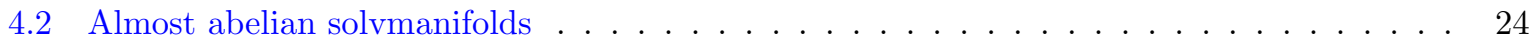

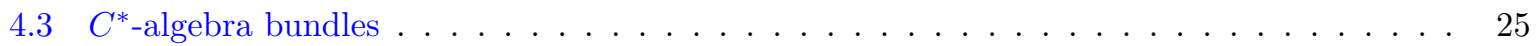

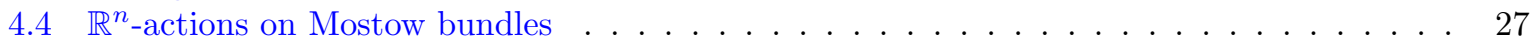

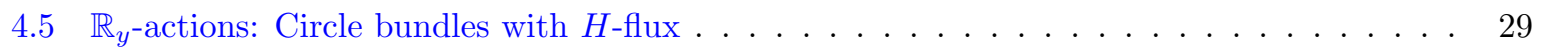

$4.6 \quad \mathbb{R}_{z}$-actions: noncommutative torus bundles $\ldots \ldots \ldots \ldots \ldots \ldots \ldots \ldots$

5 Three-dimensional solvmanifolds and their T-duals $\mathbf{3 5}$

5.1 Mostow bundles and $\mathrm{SL}(2, \mathbb{R})$ conjugacy classes $\ldots \ldots \ldots \ldots \ldots$

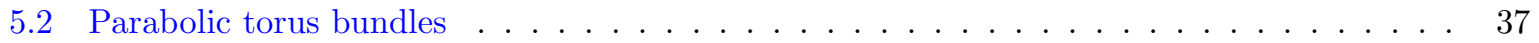

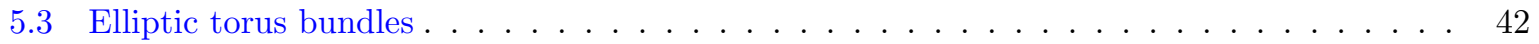

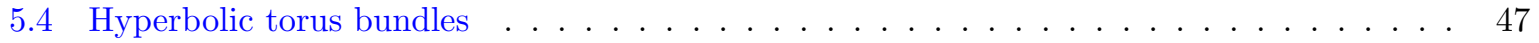

$\begin{array}{ll}\text { References } & 49\end{array}$

\section{Introduction}

\section{$1.1 \quad$ Background}

T-duality is a symmetry of string theory which relates distinct spaces that describe the same physics. It has presented a challenge to mathematics in finding a rigorous framework in which these 'equivalences' of spaces is manifest. It was realized early on that noncommutative geometry provides such a framework [18, 30], at least in the simplest cases of tori endowed with a trivial gerbe, where subsequently it was shown that T-duality is realised as Morita equivalence of noncommutative tori $[5,29,38,47,48]$.

T-duality of spaces which are compactified on tori, or more generally torus bundles, can be explained topologically in terms of correspondence spaces which implement a smooth analog of the Fourier-Mukai transform [24]. In the correspondence space picture, T-duality transformations are realised as homeomorphisms in the mapping class group of the fibres of doubled torus bundles. This gives rise to an isomorphism of K-theory groups, which are the groups of D-brane charges on the pertinent space; as this only concerns how topological data of the space change under T-duality, it is commonly refered to as 'topological T-duality', to distinguish it from the more physical notion of T-duality which also dictates how geometric data on the space should transform. It was shown by [32] that this can be reformulated in terms of the $C^{*}$-algebra of functions on the space by considering its crossed product by an action of the abelian Lie group $\mathbb{R}^{n}$, leading to a general T-duality formalism that can be regarded as a noncommutative version of the topological aspects of the Fourier-Mukai transform; this version of T-duality is often called the ' $C^{*}$-algebraic formulation' of topological T-duality.

The story becomes more interesting for spaces that are endowed with a non-trivial gerbe, which in string theory typically comprise torus bundles with ' $H$-flux'. The gerbe can be encoded in the data of a continuous-trace $C^{*}$-algebra with a non-trivial Dixmier-Douady class, which is a noncommutative algebra to which the formalism of topological T-duality was applied originally in $[3,32,33]$. In addition to relating spaces with different topologies, T-duality in string theory for such instances predicts the existence of 'non-geometric' spaces, called T-folds [25], which cannot be viewed as conventional Hausdorff topological spaces. In these instances the 
correspondence space picture 'geometrizes' the action of T-duality. It was shown by $[4,32,33]$ that the T-folds of [25] have a rigorous incarnation in noncommutative geometry as $C^{*}$-algebra bundles of noncommutative tori; necessary and sufficient conditions for the existence of 'classical' T-dual Hausdorff spaces were developed in terms of topological data, and explicit constructions of 'non-classical' T-duals as noncommutative torus bundles were given. These points of view were harmonised in [6], and in [7] a $C^{*}$-algebraic version of the correspondence space construction was given. The explicit connections of these noncommutative torus bundles to the T-folds of [25] in the setting of noncommutative gauge theories on D-branes in T-folds was elucidated in [16, 19, 28, 31]. Topological T-duality and T-folds have also been studied rigorously from other approaches based on homotopy theory [8,9] and on higher geometry [36].

In string theory, the simplest examples of torus bundles are sometimes called 'twisted tori' [13]; although this name is a misnomer, we continue to use it as it is convenient for our purposes. These are fibrations of $n$-dimensional tori $\mathbb{T}^{n}$ over a circle $\mathbb{T}$ which do not carry the extra data of a gerbe; they have monodromy in the mapping class group $\operatorname{SL}(n, \mathbb{Z})$ of the torus fibers. The simplest examples of these, the Heisenberg nilmanifolds, are T-dual to torus bundles with $H$-flux and also to T-folds, and they arise in the $C^{*}$-algebraic constructions of $[32,33]$. However, there are other examples which do not have any classical dual with $H$-flux, and these are missed by the usual $C^{*}$-algebraic framework which starts from continuous-trace algebras. The simplest examples of these with $n=2$ were studied in [28] in the language of noncommutative gauge theories, where it was shown that the monodromy of the original torus bundles becomes a non-trivial Morita equivalence of the fiber noncommutative tori of the dual $C^{*}$-algebra bundle. As far as we are aware, these are new examples of noncommutative torus bundles which have not been rigorously studied in the mathematics literature, and the primary purpose of this paper is to fill this gap: starting from the $C^{*}$-algebra of functions on a twisted torus in any dimension, we give a rigorous construction of the topological T-duals in the $C^{*}$-algebraic framework and precisely describe the non-classical $C^{*}$-algebra bundles with their Morita equivalence monodromies. This includes some of the examples from $[32,33]$ based on topological T-duality applied to continuoustrace algebras, and the examples of [28] based on T-duality in noncommutative gauge theory, while at the same time it produces many new examples. In particular, we give a unified description of the noncommutative torus bundles which are T-dual to twisted tori in any dimension.

The noncommutative gauge theory on a D-brane comes with other moduli, in addition to the noncommutativity parameters, which also generally transform in a non-trivial way under the monodromies so as to leave the physics unchanged [28]. In the absence of other moduli, as in topological T-duality, the non-trivial Morita equivalences of the fibres of the $C^{*}$-algebra bundles require an interpretation akin to the topological monodromies, which act as homeomorphisms in the mapping class group $\operatorname{SL}(n, \mathbb{Z})$ of the fibres of the original twisted torus. This is naturally achieved by considering our $C^{*}$-algebras as objects in a category where both the usual *-isomorphisms as well as Morita equivalences are realised as isomorphisms. This category is well-known to experts and all of our considerations of topological T-duality in this paper will take place therein. This perspective will also be advantageous for eventual rigorous considerations of noncommutative gauge theories on these $C^{*}$-algebra bundles in terms of projective modules, as well as for the treatments of D-branes in terms of their K-theory, though we do not pursue these further aspects in the present paper.

\subsection{Summary and outline}

In this paper our starting point is a very general definition of a twisted torus $\mathbb{T}_{\Lambda_{G}}$ as the quotient of a locally compact group $\mathrm{G}$ by a lattice $\Lambda_{\mathrm{G}}$ in $\mathrm{G}$; this definition encompasses the $\mathbb{T}^{n}$-bundles over $\mathbb{T}$ discussed above, along with many other known examples from string theory. We regard $\mathbb{T}_{\Lambda_{\mathrm{G}}}$ as a 'torus bundle without $H$-flux', which is captured simply by the $C^{*}$-algebra of 
functions $C\left(\mathbb{T}_{\Lambda_{\mathrm{G}}}\right)$. This is ultimately the novelty of our approach, which leads to a simpler perspective on topological T-duality as compared to the approach of $[32,33]$ based on the more complicated continuous-trace algebras. Our approach uses similar techniques as those of $[32,33]$ for evaluating Morita equivalences of cross products by actions of $\mathbb{R}^{n}$, though with a much simpler $C^{*}$-algebraic structure. In particular, in this paper we do not develop any new $C^{*}$-algebraic machinery as such, but instead we gather a fortuitously existing collection of results that enable us to explicitly identify both classical and non-classical T-duals of twisted tori with relatively straightforward algebraic techniques. On the other hand, the tradeoff for the simplicity of our framework is the absence of some key constructions from [4, 32, 33].

We have endeavoured throughout to provide a fairly self-contained, and at times pedagogical, presentation. For this reason we have collected all the key concepts and tools involving cross products of $C^{*}$-algebras and Morita equivalence in Section 2. Experts versed in $C^{*}$-algebra theory may safely skip this section.

In Section 3 we give our definition of twisted tori $\mathbb{T}_{\Lambda_{\mathrm{G}}}$ and discuss the $C^{*}$-algebraic formulation of topological T-duality that we employ in this paper. We describe how T-dual $C^{*}$-algebras are naturally isomorphic when regarded as objects of the additive category $\mathscr{K} \mathscr{K}$ that underlies Kasparov's bivariant K-theory, and we adapt the construction of noncommuative correspondences from [7] as diagrams in this category. We spell out some simple techniques that we use to compute classical T-duals with $H$-flux, i.e., the T-dual algebra is a certain continuous-trace $C^{*}$ algebra with non-trivial Dixmier-Douady class, and more general techniques based on Green's symmetric imprimitivity theorem which enable the computation of noncommutative T-duals. We illustrate our scheme on two well-known examples which have classical T-duals: we reproduce the standard rules for T-duality of tori as well as the topology changing mechanism for T-duality of orbifolds of compact Lie groups $\mathrm{G}$.

In Section 4 we come to the main class of examples and results of this paper. We review the definition and topology of the special class of twisted tori provided by almost abelian solvmanifolds, which are $\mathbb{T}^{n}$-bundles over a circle $\mathbb{T}$. Accordingly, we regard the algebra of functions $C\left(\mathbb{T}_{\Lambda_{\mathrm{G}}}\right)$ as an object in the category $\mathcal{R} \mathscr{K}_{\mathbb{T}}$ of $C^{*}$-algebra bundles over $\mathbb{T}$, where in particular fibrewise Morita equivalences are isomorphisms. T-duality in this category requires fibrewise actions of $\mathbb{R}^{n}$, and in particular $\mathbb{R}^{n}$-actions which act non-trivially on the base $\mathbb{T}$ would take the algebra out of the category $\mathcal{R} \mathscr{K}_{\mathbb{T}}$. This means that the 'essentially doubled spaces' of [28], which arise from T-duality along the base circle and require a completely doubled formalism, are not considered in this paper; they require working in a different category, which we do not discuss here. We give necessary and sufficient criteria for the existence of classical T-duals with $H$-flux in this case which are based on simple algebraic data of the underlying group $\mathrm{G}$, and we explicitly compute the corresponding continuous-trace $C^{*}$-algebras dual to any almost abelian solvmanifold $\mathbb{T}_{\Lambda_{G}}$ satisfying these conditions. We further show that any such solvmanifold has a non-classical T-dual that is a $C^{*}$-algebra bundle of noncommutative $n$-tori over $\mathbb{T}$, which we also compute explicitly; this rigorously confirms, in particular, arguments from string theory suggesting that non-geometric solutions result from T-duality on some six-dimensional almost abelian solvmanifolds [1].

Finally, Section 5 is devoted to explicit examples of the general formalism of Section 4 . We apply our results to all three classes of three-dimensional solvmanifolds. We recover in this way a new perspective on the well-known T-duals of the Heisenberg nilmanifolds: the three-torus $\mathbb{T}^{3}$ with $H$-flux, and the basic noncommutative principal $\mathbb{T}^{2}$-bundle over $\mathbb{T}$ given by the group $C^{*}$ algebra of the integer Heisenberg group. Our general formalism also rigorously reproduces the noncommutative torus bundles from [28] which are T-dual to Euclidean solvmanifolds for the $\mathbb{Z}_{4}$ elliptic conjugacy class of $\mathrm{SL}(2, \mathbb{Z})$. We extend these results to give new examples of noncommutative torus bundles dual to Euclidean solvmanifolds for the $\mathbb{Z}_{2}$ and $\mathbb{Z}_{6}$ elliptic conjugacy classes, as well as to the Poincaré solvmanifolds. In particular, our formalism extends the $C^{*}$-algebraic 
formulation of topological T-duality to the case of non-principal torus bundles, which have also been previously considered in [22].

\section{Crossed products and duality}

In this section we summarise some of the mathematical tools that we will use in this paper. A good reference for the material covered in the following is the book [52]. Throughout this paper, all topological spaces are assumed to be second countable (hence separable), locally compact and Hausdorff.

\subsection{Dynamical systems and their crossed products}

Let $\mathrm{G}$ be a locally compact group and let $X$ be a $\mathrm{G}$-space, i.e., a topological space which is acted upon homeomorphically by $\mathrm{G}$; we denote the $\mathrm{G}$-action $\mathrm{G} \times X \rightarrow X$ by $(\gamma, x) \mapsto \gamma \cdot x$. The pair $(X, \mathrm{G})$ is called a transformation group. A related concept is that of a dynamical system, which is a triple $(\mathcal{A}, \mathrm{G}, \alpha)$ consisting of an algebra $\mathcal{A}$ and a locally compact group $\mathrm{G}$ acting on $\mathcal{A}$ via a group homomorphism $\alpha: \mathrm{G} \rightarrow \operatorname{Aut}(\mathcal{A})$, denoted $\gamma \mapsto\left(\alpha_{\gamma}: \mathcal{A} \rightarrow \mathcal{A}\right)$ for $\gamma \in \mathrm{G}$. In topological T-duality one usually requires $\mathcal{A}$ to be a $C^{*}$-algebra, in which case $(\mathcal{A}, \mathrm{G}, \alpha)$ is called a $C^{*}$-dynamical system. Two dynamical systems $(\mathcal{A}, \mathrm{G}, \alpha)$ and $(\mathcal{B}, \mathrm{G}, \beta)$ are equivalent if there is an algebra isomorphism $\varphi: \mathcal{A} \rightarrow \mathcal{B}$ which intertwines the G-actions: $\varphi \circ \alpha_{\gamma}=\beta_{\gamma} \circ \varphi$ for all $\gamma \in \mathrm{G}$.

If $\mathcal{A}$ is a commutative $C^{*}$-algebra, then we call $(\mathcal{A}, \mathrm{G}, \alpha)$ a commutative dynamical system. In that case, by Gelfand duality $\mathcal{A}=C_{0}(X)$ is the algebra of continuous functions vanishing at infinity on a topological space $X$ equipped with the G-action $\left.\alpha_{\gamma}^{\dagger}\right|_{X}$ for $\gamma \in \mathrm{G}$ under the identification of points $x \in X$ with irreducible representations of $C_{0}(X)$, which are onedimensional and given by the point evaluation maps $\mathrm{ev}_{x}: \mathcal{A} \rightarrow \mathcal{A}$ with $\operatorname{ev}_{x}(\mathrm{f})=\mathrm{f}(x)$; then $(X, \mathrm{G})$ is a transformation group. Conversely, given a transformation group $(X, \mathrm{G})$, there is an associated commutative dynamical system $\left(C_{0}(X), \mathrm{G}, \alpha\right)$, where $\alpha_{\gamma}(\mathrm{f})(x)=\mathrm{f}\left(\gamma^{-1} \cdot x\right)$ for $\gamma \in \mathrm{G}, \mathrm{f} \in C_{0}(X)$ and $x \in X$. In other words, there is a one-to-one correspondence between transformation groups and commutative $C^{*}$-dynamical systems.

If the $C^{*}$-algebra $\mathcal{A}$ is not commutative, then we call $(\mathcal{A}, \mathrm{G}, \alpha)$ a noncommutative $C^{*}$ dynamical system.

As usual, it is more useful to work with a representation rather than the abstract dynamical system itself. A covariant representation of a dynamical system $(\mathcal{A}, \mathrm{G}, \alpha)$ in a $C^{*}$-algebra $\mathcal{B}$ with multiplier algebra $\mathrm{M}(\mathcal{B})$ is a pair $(\Pi, U)$ consisting of a homomorphism $\Pi: \mathcal{A} \rightarrow \mathrm{M}(\mathcal{B})$ and a unitary representation $U: \mathrm{G} \rightarrow \mathrm{M}(\mathcal{B}), \gamma \mapsto U_{\gamma}$ which satisfy the compatibility condition

$$
\Pi\left(\alpha_{\gamma}(a)\right)=U_{\gamma} \Pi(a) U_{\gamma}^{-1}
$$

for all $\gamma \in \mathrm{G}$ and $a \in \mathcal{A}$. A natural choice is to take $\mathcal{B}=\mathcal{K}(\mathcal{H})$ to be the $C^{*}$-algebra of compact operators on a separable Hilbert space $\mathcal{H}$, which gives a representation $\Pi: \mathcal{A} \rightarrow \mathrm{B}(\mathcal{H})$ of the algebra $\mathcal{A}$ by bounded operators $\mathrm{B}(\mathcal{H})$ on $\mathcal{H}$ and a unitary representation $U: \mathrm{G} \rightarrow \mathrm{B}(\mathcal{H})$ of the group $\mathrm{G}$ on $\mathcal{H}$; in this case we call $(\Pi, U)$ a covariant representation of $(\mathcal{A}, \mathrm{G}, \alpha)$ on $\mathcal{H}$.

When a group $\mathrm{G}$ acts on a space $X$, one is naturally interested in considering the quotient space $X / \mathrm{G}$ of $\mathrm{G}$-orbits on $X$. When $\mathrm{G}$ acts freely and properly on $X$, this is described algebraically by the algebra of functions $C_{0}(X / \mathrm{G})$. More generally, the subalgebra of G-invariant elements $\mathcal{A}^{\mathrm{G}} \subseteq \mathcal{A}$ of a $\mathrm{G}$-algebra $\mathcal{A}$ can be used to represent the quotient, even for $\mathrm{G}$-actions with fixed points. A more general and systematic way of dealing with the effective algebraic "quotient" is through the crossed product algebra $\mathcal{A} \rtimes_{\alpha} \mathrm{G}$ for a dynamical system $(\mathcal{A}, \mathrm{G}, \alpha)$. For a transformation group $(X, \mathrm{G})$, this description is particularly powerful in the cases where the 
quotient $X / \mathrm{G}$ is not a Hausdorff space, while for a free and proper G-action it gives an algebra with the same spectrum $X / \mathrm{G}$ as the algebra $C_{0}(X / \mathrm{G})=C_{0}(X)^{\mathrm{G}}$ of $\mathrm{G}$-invariant functions on $X$.

In order to define the crossed product algebra, we first define

$$
\|f\|_{\text {univ }}:=\sup _{(\Pi, U)}\left\|\left(\Pi \rtimes_{\alpha} U\right)(f)\right\|
$$

for compactly supported functions $f \in C_{\mathrm{c}}(\mathrm{G}, \mathcal{A})$, where the supremum is taken over (possibly degenerate) covariant representations $(\Pi, U)$ of $(\mathcal{A}, \mathrm{G}, \alpha)$ with

$$
\left(\Pi \rtimes_{\alpha} U\right)(f):=\int_{\mathrm{G}} \Pi(f(\gamma)) U_{\gamma} \mathrm{d} \mu_{\mathrm{G}}(\gamma),
$$

and $\mu_{\mathrm{G}}$ denotes the left invariant Haar measure on $\mathrm{G}$. This defines a norm, called the universal norm, on the space $C_{\mathrm{c}}(\mathrm{G}, \mathcal{A})$. Then the crossed product algebra $\mathcal{A} \rtimes_{\alpha} \mathrm{G}$ is the completion (in the universal norm) of the algebra $C_{\mathrm{c}}(\mathrm{G}, \mathcal{A})$ equipped with the convolution product

$$
\left(f \star f^{\prime}\right)(\gamma):=\int_{\mathrm{G}} f\left(\gamma^{\prime}\right) \alpha_{\gamma^{\prime}}\left(f^{\prime}\left(\gamma^{\prime-1} \gamma\right)\right) \mathrm{d} \mu_{\mathrm{G}}\left(\gamma^{\prime}\right),
$$

for all $f, f^{\prime}: \mathrm{G} \rightarrow \mathcal{A}$. In general this is a noncommutative multiplication, even for commutative dynamical systems. Since $\mathcal{A}$ is a $C^{*}$-algebra, there is a $*$-structure on the convolution algebra defined by

$$
f^{\dagger}(\gamma):=\Delta_{\mathrm{G}}(\gamma)^{-1} \alpha_{\gamma}\left(f\left(\gamma^{-1}\right)^{*}\right)
$$

where $\Delta_{\mathrm{G}}: \mathrm{G} \rightarrow \mathbb{R}^{+}$is the modular function of $\mathrm{G}$ defined through

$$
\Delta_{\mathrm{G}}\left(\gamma^{\prime}\right) \int_{\mathrm{G}} f(\gamma) \mathrm{d} \mu_{\mathrm{G}}(\gamma)=\int_{\mathrm{G}} f\left(\gamma \gamma^{\prime}\right) \mathrm{d} \mu_{\mathrm{G}}(\gamma)
$$

for $f \in C_{\mathrm{c}}(\mathrm{G}, \mathcal{A})$ and $\gamma^{\prime} \in \mathrm{G}$. By the uniqueness of the left invariant Haar measure $\mu_{\mathrm{G}}$ up to a positive constant, $\Delta_{\mathrm{G}}\left(\gamma^{\prime}\right)$ is independent of $f$ and $\Delta_{\mathrm{G}}$ is easily proven to be a continuous group homomorphism from $\mathrm{G}$ to the multiplicative group $\mathbb{R}^{+}$; it is trivial for abelian groups and for compact groups.

When $\mathcal{A}=C_{\mathrm{c}}(X)$ is the algebra of a commutative dynamical system, the convolution algebra $C_{\mathrm{c}}(\mathrm{G} \times X)$ consists of functions $f: \mathrm{G} \times X \rightarrow \mathbb{C}$ and the convolution product reads as

$$
\left(f \star f^{\prime}\right)(\gamma, x)=\int_{\mathrm{G}} f\left(\gamma^{\prime}, x\right) f^{\prime}\left(\gamma^{\prime-1} \gamma, \gamma^{\prime-1} \cdot x\right) \mathrm{d} \mu_{\mathrm{G}}\left(\gamma^{\prime}\right),
$$

while the $*$-algebra structure is given by

$$
f^{\dagger}(\gamma, x)=\Delta_{\mathrm{G}}(\gamma)^{-1} \overline{f\left(\gamma^{-1}, \gamma^{-1} \cdot x\right)}
$$

The crossed product is a generalization of the usual group algebra $C^{*}(\mathrm{G})$, the completion (in the universal norm) of $C_{\mathrm{c}}(\mathrm{G})$ which is recovered in the case $\mathcal{A}=\mathbb{C}$ (the $C^{*}$-algebra of a point) wherein $\alpha_{\gamma}=\operatorname{id}_{\mathcal{A}}: \mathcal{A} \rightarrow \mathcal{A}$ for all $\gamma \in \mathrm{G}$ and (2.1) recovers the usual convolution product of functions on the group G. As explained in Section 2.2 below, the group $C^{*}$-algebra description illustrates the relation between crossed products and semi-direct products of groups (see Theorem 2.5). We also note that if a group $\mathrm{G}$ acts trivially on an algebra $\mathcal{A}$, then $\mathcal{A} \rtimes \mathrm{G} \simeq \mathcal{A} \otimes C^{*}(\mathrm{G})$.

The crossed product can be thought of as a universal object for covariant representations of the dynamical system $(\mathcal{A}, \mathrm{G}, \alpha)$, in the following sense: Define the universal covariant representation $(\boldsymbol{\Pi}, \boldsymbol{U})$ of $(\mathcal{A}, \mathrm{G}, \alpha)$ in $\mathcal{A} \rtimes_{\alpha} \mathrm{G}$ by

$$
(\boldsymbol{\Pi}(a) f)(\gamma)=a f(\gamma) \quad \text { and } \quad\left(\boldsymbol{U}_{\gamma^{\prime}} f\right)(\gamma)=\alpha_{\gamma^{\prime}}\left(f\left(\gamma^{\prime-1} \gamma\right)\right)
$$


for $a \in \mathcal{A}, f \in C_{\mathrm{c}}(\mathrm{G}, \mathcal{A})$ and $\gamma, \gamma^{\prime} \in \mathrm{G}$. Then the universal property defining the crossed product implies that any covariant representation $(\Pi, U)$ of $(\mathcal{A}, \mathrm{G}, \alpha)$ in a $C^{*}$-algebra $\mathcal{B}$ factors through the universal covariant representation: There exists a unique homomorphism $\varphi: \mathrm{M}\left(\mathcal{A} \rtimes_{\alpha} \mathrm{G}\right) \rightarrow \mathrm{M}(\mathcal{B})$ such that

$$
\Pi=\varphi \circ \Pi \quad \text { and } \quad U_{\gamma}=\varphi\left(U_{\gamma}\right)
$$

for all $\gamma \in \mathrm{G}$.

If $(\Pi, U)$ is a covariant representation of the dynamical system $(\mathcal{A}, \mathrm{G}, \alpha)$ on a Hilbert space $\mathcal{H}$, then

$$
\Phi_{(\Pi, U)}(f):=\left(\Pi \rtimes_{\alpha} U\right)(f)
$$

defines a representation $\Phi_{(\Pi, U)}: C_{\mathrm{c}}(\mathrm{G}, \mathcal{A}) \rightarrow \mathrm{B}(\mathcal{H})$ of the crossed product $\mathcal{A} \rtimes_{\alpha} \mathrm{G}$ as bounded operators on $\mathcal{H}$. This is called the integrated form of the covariant representation $(\Pi, U)$. In particular, it maps the convolution product $(2.1)$ onto the operator product in the algebra $\mathrm{B}(\mathcal{H})$,

$$
\Phi_{(\Pi, U)}(f \star g)=\Phi_{(\Pi, U)}(f) \Phi_{(\Pi, U)}(g),
$$

and it is covariant in the sense that

$$
\Phi_{(\Pi, U)}\left(i_{\mathrm{G}}(\gamma)(f)\right)=U_{\gamma} \Phi_{(\Pi, U)}(f),
$$

where $\left(i_{\mathrm{G}}(\gamma)(f)\right)\left(\gamma^{\prime}\right):=\alpha_{\gamma}\left(f\left(\gamma^{-1} \gamma^{\prime}\right)\right)$ for each $\gamma, \gamma^{\prime} \in \mathrm{G}$ and $f \in C_{\mathrm{c}}(\mathrm{G}, \mathcal{A})$.

Example 2.1 (noncommutative two-tori). The noncommutative torus is a fundamental example of a noncommutative space in both physics and mathematics. Its original incarnation [43] is a nice example of a crossed product construction, which will play a fundamental role later on in this paper. Let $\left(C(\mathbb{T}), \mathbb{Z}, \tau^{\theta}\right)$ be the commutative $C^{*}$-dynamical system where $\tau^{\theta}$ is induced through pullback by rotations of the circle $\mathbb{T}$ through a fixed angle $\theta \in \mathbb{R} / \mathbb{Z}$ :

$$
\tau_{n}^{\theta}(\mathrm{f})(z)=\mathrm{f}\left(\mathrm{e}^{2 \pi \mathrm{i} n \theta} z\right)
$$

for $n \in \mathbb{Z}, \mathbf{f} \in C(\mathbb{T})$ and $z \in \mathbb{T}$. The resulting crossed product

$$
\mathrm{A}_{\theta}:=C(\mathbb{T}) \rtimes_{\tau^{\theta}} \mathbb{Z}
$$

is a called a rotation algebra, and for irrational values of $\theta$ it can be identified as a noncommutative two-torus $\mathbb{T}_{\theta}^{2}$ in the following way.

By definition, the algebra $A_{\theta}$ is the universal norm completion of the convolution algebra $C_{\mathrm{c}}\left(\mathbb{Z} \times \mathbb{T}\right.$ ), whose elements $f=\left\{f_{n}\right\}_{n \in \mathbb{Z}}$ can be regarded as sequences (with only finitely many nonvanishing terms) of functions $f_{n}: \mathbb{T} \rightarrow \mathbb{C}$. The convolution product is given by

$$
\left(f \star_{\theta} g\right)_{n}(z):=\sum_{n^{\prime} \in \mathbb{Z}} f_{n^{\prime}}(z) g_{n-n^{\prime}}\left(\mathrm{e}^{2 \pi \mathrm{i} n^{\prime} \theta} z\right),
$$

and the $*$-algebra structure is

$$
f_{n}^{\dagger}(z):=\overline{f_{-n}\left(\mathrm{e}^{2 \pi \mathrm{i} n \theta} z\right)} .
$$

Via the Fourier transformation

$$
f(z, w):=\sum_{n \in \mathbb{Z}} f_{n}(z) w^{n}
$$


for $w \in \mathbb{T}$, we may regard the convolution algebra $C_{\mathrm{c}}(\mathbb{Z} \times \mathbb{T})$ as a subspace of the space of functions $C\left(\mathbb{T}^{2}\right)$ equipped with the star-product

$$
\left(f \star_{\theta} g\right)(z, w)=\sum_{n \in \mathbb{Z}}\left(f \star_{\theta} g\right)_{n}(z) w^{n} .
$$

After a further Fourier transformation

$$
f_{n}(z)=\sum_{m \in \mathbb{Z}} f_{m, n} z^{m}
$$

and some simple redefinitions of the Fourier series involved, the star-product (2.2) may be written in the form

$$
\left(f \star_{\theta} g\right)(z, w)=\sum_{(m, n) \in \mathbb{Z}^{2}}\left(\sum_{\left(m^{\prime}, n^{\prime}\right) \in \mathbb{Z}^{2}} f_{m^{\prime}, n^{\prime}} g_{m-m^{\prime}, n-n^{\prime}} \mathrm{e}^{2 \pi \mathrm{i}\left(m-m^{\prime}\right) n^{\prime} \theta}\right) z^{m} w^{n} .
$$

This recovers the usual commutative pointwise multiplication of functions in $C\left(\mathbb{T}^{2}\right)$ for $\theta=0$. For $\theta \neq 0$ it realizes the irrational rotation algebra $A_{\theta}$ as a deformation of the algebra of functions $C\left(\mathbb{T}^{2}\right)$ on a two-dimensional torus $\mathbb{T}^{2}$; it is equivalent to the usual strict deformation quantization of $\mathbb{T}^{2}$ whose star-product is a twisted convolution product on $C\left(\mathbb{T}^{2}\right)$.

In the language of covariant representations of the dynamical system $\left(C(\mathbb{T}), \mathbb{Z}, \tau^{\theta}\right)$, the crossed product $\mathrm{A}_{\theta}$ is the universal $C^{*}$-algebra generated by two unitaries $U$ and $V$ satisfying the relation [52, Proposition 2.56]

$$
U V=\mathrm{e}^{-2 \pi \mathrm{i} \theta} V U \text {. }
$$

A concrete representation of $A_{\theta}$ on the Hilbert space $\mathcal{H}=L^{2}(\mathbb{T})$ is given by defining

$$
U(\mathrm{f})(z)=z \mathrm{f}(z) \quad \text { and } \quad V(\mathrm{f})(z)=\mathrm{f}\left(\mathrm{e}^{2 \pi \mathrm{i} \theta} z\right) .
$$

Example 2.2 (Noncommutative $\boldsymbol{d}$-tori). The natural higher-dimensional generalization of Example 2.1 involves a skew-symmetric real $d \times d$ matrix $\Theta=\left(\theta_{i j}\right)$, see [45]. Then the noncommutative $d$-torus $\mathrm{A}_{\Theta}=\mathbb{T}_{\Theta}^{d}$ is the universal $C^{*}$-algebra generated by $d$ unitaries $U_{1}, \ldots, U_{d}$ satisfying the relations

$$
U_{i} U_{j}=\mathrm{e}^{-2 \pi \mathrm{i} \theta_{i j}} U_{j} U_{i}
$$

for $i, j=1, \ldots, d$. By [37, Lemma 1.5], every noncommutative torus $\mathbb{T}_{\Theta}^{d}$ can be obtained as an iterated crossed product by $\mathbb{Z}$ in the following way. Let $\Theta_{\mid d-1}=\left(\theta_{i j}\right)_{1 \leq i, j \leq d-1}$, and let $U_{1}, \ldots, U_{d-1}$ be the standard generators of $A_{\Theta_{\mid d-1}}=\mathbb{T}_{\Theta_{\mid d-1}}^{d-1}$. Define a group homomorphism $\tau^{\vec{\theta}}: \mathbb{Z} \rightarrow \operatorname{Aut}\left(\mathrm{A}_{\Theta_{\mid d-1}}\right)$ by

$$
\tau_{n}^{\vec{\theta}}\left(U_{i}\right)=\mathrm{e}^{2 \pi \mathrm{in} \theta_{i d}} U_{i}
$$

for $n \in \mathbb{Z}$, where $\vec{\theta}:=\left(\theta_{i d}\right) \in \mathbb{R}^{d-1}$. Then there is an isomorphism of $C^{*}$-algebras

$$
\mathrm{A}_{\Theta} \simeq \mathrm{A}_{\Theta_{\mid d-1}} \rtimes_{\tau^{\vec{\theta}}} \mathbb{Z}
$$

In the particular case where $\Theta_{\mid d-1}=\mathbf{0}_{d-1}$, we denote the corresponding noncommutative $d$-torus by $A_{\vec{\theta}}=\mathbb{T}_{\vec{\theta}}^{d}$, and (2.4) shows that it can be obtained by a crossed product of the commutative algebra of functions on a $d-1$-torus by an action of $\mathbb{Z}$ :

$$
\mathrm{A}_{\vec{\theta}} \simeq C\left(\mathbb{T}^{d-1}\right) \rtimes_{\tau^{\vec{\theta}}} \mathbb{Z} .
$$




\subsection{Semi-direct products and group algebras}

Most of our considerations later on will focus on spaces that can be obtained from semi-direct products of groups. We will now explain the relation between crossed products and semi-direct products which will be useful for these examples.

There are two ways to think about the semi-direct product construction:

(1) Let $\mathrm{G}$ be a group with two subgroups $\mathrm{N}$ and $\mathrm{H}$ such that $\mathrm{N}$ is normal. If $\mathrm{N} \cap \mathrm{H}=\{e\} \subset \mathrm{G}$ and every element of $\mathrm{G}$ can be written as a product of an element of $\mathrm{N}$ with an element of $\mathrm{H}$, then we say that $\mathrm{G}$ is a semi-direct product of its subgroups $\mathrm{N}$ and $\mathrm{H}$ and we write $\mathrm{G}=\mathrm{NH}$.

(2) Let $\mathrm{N}$ and $\mathrm{H}$ be two groups together with a left action $\varphi: \mathrm{H} \rightarrow \operatorname{Aut}(\mathrm{N})$ of $\mathrm{H}$ on $\mathrm{N}$ by automorphisms, which we denote by $\varphi_{h}(n)={ }^{h} n$ for $h \in \mathrm{H}$ and $n \in \mathrm{N}$; in particular ${ }^{h}\left(n n^{\prime}\right)={ }^{h} n^{h} n^{\prime}$. We write ${ }^{\mathrm{H}} \mathrm{N}$ to indicate that $\mathrm{H}$ acts on $\mathrm{N}$ from the left. The semi-direct product of $\mathrm{N}$ and $\mathrm{H}$ is the group $\mathrm{N} \rtimes_{\varphi} \mathrm{H}$ defined to be the set $\mathrm{N} \times \mathrm{H}$ with the product

$$
(n, h)\left(n^{\prime}, h^{\prime}\right)=\left(n^{h} n^{\prime}, h h^{\prime}\right) .
$$

The inverse is then $(n, h)^{-1}=\left(h^{-1} n^{-1}, h^{-1}\right)$.

These two definitions are equivalent: Given subgroups $\mathrm{N}, \mathrm{H} \subset \mathrm{G}$ as in point (1), it follows that $\mathrm{G} \simeq \mathrm{N} \rtimes_{\mathrm{Ad}} \mathrm{H}$ where $\mathrm{Ad}$ is the adjoint action: $\operatorname{Ad}_{h}(n)=h n h^{-1}$. On the other hand, every element of the group $\mathrm{G}=\mathrm{N} \rtimes_{\varphi} \mathrm{H}$ defined in (2) can be written as $(n, h)=\left(n, e_{\mathrm{H}}\right)\left(e_{\mathrm{N}}, h\right)$ and the subgroups $\mathrm{N} \times\left\{e_{\mathrm{H}}\right\}$ and $\left\{e_{\mathrm{N}}\right\} \times \mathrm{H}$ intersect only in the identity of $\mathrm{G}$. If the action of $\mathrm{H}$ on $\mathrm{N}$ is trivial, i.e., $\varphi_{h}=\mathrm{id}_{\mathrm{N}}$ for all $h \in \mathrm{H}$, then the semi-direct product reduces to the direct product $\mathrm{N} \rtimes_{\varphi} \mathrm{H}=\mathrm{N} \times \mathrm{H}$.

Later on we will need to consider the interplay between semi-direct products and quotient groups, which is provided by the simple

Lemma 2.3. Let $\mathrm{G}=\mathrm{N} \rtimes_{\varphi} \mathrm{H}$ be a semi-direct product, and let $\mathrm{V} \subset \mathrm{N}$ be a subgroup which is normal in $\mathrm{G}$. Then the quotient group $\mathrm{G} / \mathrm{V}$ is the semi-direct product $(\mathrm{N} / \mathrm{V}) \rtimes_{\varphi} \vee \mathrm{H}$, where $\varphi^{\vee}$ is the action $\varphi$ of $\mathrm{H}$ induced on the quotient group $\mathrm{N} / \mathrm{V}$.

If $\mathrm{N}$ is a group, we write $C^{*}(\mathrm{~N})$ for the corresponding group $C^{*}$-algebra, i.e., for the crossed product $\mathbb{C} \rtimes \mathrm{N}$. If $\mathrm{N}$ is finite, then $C^{*}(\mathrm{~N})=\mathbb{C}[\mathrm{N}]$ is the linear space freely generated over $\mathbb{C}$ by the group elements, made into an algebra by linearly extending the product from $\mathrm{N}$ to $\mathbb{C}[\mathrm{N}]$; equivalently it is the algebra of continuous functions on $\mathrm{N}$ with the convolution product. Given a left $\mathrm{H}$-action $\varphi: \mathrm{H} \rightarrow \operatorname{Aut}(\mathrm{N})$, there is an induced action $\varphi^{*}: \mathrm{H} \rightarrow \operatorname{Aut}\left(C^{*}(\mathrm{~N})\right)$ via pullback. For $\mathrm{H}$ and $\mathrm{N}$ finite the vector spaces $C^{*}(\mathrm{~N}) \times \mathrm{H}$ and $C^{*}(\mathrm{~N} \times \mathrm{H})$ are canonically isomorphic, and it is straightforward to show that the corresponding crossed product and semi-direct product are related by

Proposition 2.4. If $\mathrm{N}$ and $\mathrm{H}$ are finite groups, then

$$
C^{*}(\mathrm{~N}) \rtimes_{\varphi^{*}} \mathrm{H} \simeq C^{*}\left(\mathrm{~N} \rtimes_{\varphi} \mathrm{H}\right) .
$$

Proof. Note that $C^{*}(\mathrm{~N}) \rtimes_{\varphi^{*}} \mathrm{H}=\mathbb{C}[\mathrm{N}] \rtimes_{\varphi^{*}} \mathrm{H}$ is the vector space of functions $f: \mathrm{H} \rightarrow \mathbb{C}[\mathrm{N}]$ with convolution product

$$
\left(f \star_{\mathbb{C}[\mathrm{N}] \rtimes_{\varphi^{*} \mathrm{H}}} f^{\prime}\right)(h)=\sum_{h^{\prime} \in \mathrm{H}} f\left(h^{\prime}\right) \star_{\mathbb{C}[\mathrm{N}]} \varphi_{h^{\prime}}^{*}\left(f^{\prime}\left(h^{\prime-1} h\right)\right),
$$

and using the convolution product in $\mathbb{C}[\mathrm{N}]$ this can be written as

$$
\left(f \star_{\mathbb{C}[\mathrm{N}] \rtimes_{\varphi^{*}} \mathrm{H}} f^{\prime}\right)(n, h)=\sum_{h^{\prime} \in \mathrm{H}} \sum_{n^{\prime} \in \mathrm{N}} f\left(n^{\prime}, h^{\prime}\right) f^{\prime}\left({h^{\prime-1}}\left(n^{\prime-1} n\right), h^{\prime-1} h\right),
$$

which is easily seen to coincide with the convolution product in $C^{*}\left(\mathrm{~N} \rtimes_{\varphi} \mathrm{H}\right)=\mathbb{C}\left[\mathrm{N} \rtimes_{\varphi} \mathrm{H}\right]$. 
A more general result holds if $\mathrm{N}$ and $\mathrm{H}$ are locally compact groups with $\varphi: \mathrm{H} \rightarrow \operatorname{Aut}(\mathrm{N})$ a continuous action of $\mathrm{H}$ on $\mathrm{N}$ via automorphisms (i.e., $(h, n) \mapsto \varphi_{h}(n)$ is a continuous map from $\mathrm{H} \times \mathrm{N}$ to $\mathrm{N}$ ). In this case the semi-direct product $\mathrm{N} \rtimes_{\varphi} \mathrm{H}$ is a locally compact group (in the product topology on $\mathrm{N} \times \mathrm{H}$ ) with $\mathrm{N}$ a closed normal subgroup and $\mathrm{H}$ a closed subgroup (see [52, Proposition 3.11] with $A=\mathbb{C}$ ). The analogue of item (1) above also holds in the context of locally compact groups if $\mathrm{G}$ is $\sigma$-compact, and $\mathrm{N}$ and $\mathrm{H}$ are closed subgroups of $\mathrm{G}$.

The action $\beta$ defining the $C^{*}$-dynamical system $\left(C^{*}(\mathrm{~N}), \mathrm{H}, \beta\right)$ and hence the crossed product $C^{*}(\mathrm{~N}) \rtimes_{\beta} \mathrm{H}$ is the composition of the pullback $\varphi^{*}$ of the action $\varphi: \mathrm{H} \rightarrow \operatorname{Aut}(\mathrm{N})$ with the action $\sigma_{\mathrm{H}}: \mathrm{H} \rightarrow \mathbb{R}^{+} \subset \operatorname{Aut}\left(C^{*}(\mathrm{~N})\right)$ that enters the definition of the Haar measure on $\mathrm{N} \rtimes_{\varphi} \mathrm{H}$ in terms of the Haar measures on $\mathrm{N}$ and $\mathrm{H}$ : If $\mu_{\mathrm{N}}$ is a (left invariant) Haar measure on $\mathrm{N}$, then the integral $I_{h}(F)=\int_{\mathrm{N}} F\left({ }^{h} n\right) \mathrm{d} \mu_{\mathrm{N}}(n)$ for $F \in C^{*}(\mathrm{~N})$ is left invariant, i.e., $I_{h}\left(\lambda_{n^{\prime}} F\right)=I_{h}(F)$ where $\left(\lambda_{n^{\prime}} F\right)(n):=F\left(n^{\prime-1} n\right)$ for all $n^{\prime} \in \mathbf{N}$ (use invariance of the Haar measure under $n \rightarrow h^{-1} n^{\prime}$ ). Uniqueness of the Haar measure up to a positive constant then implies there exists a function $\sigma_{\mathrm{H}}: \mathrm{H} \rightarrow \mathbb{R}^{+}$such that

$$
\sigma_{\mathrm{H}}(h) \int_{\mathrm{N}} F\left({ }^{h} n\right) \mathrm{d} \mu_{\mathrm{N}}(n)=\int_{\mathrm{N}} F(n) \mathrm{d} \mu_{\mathrm{N}}(n) .
$$

It is straighforward to see that $\sigma_{\mathrm{H}}$ is a group homomorphism and that it is continuous [52, Section 2]. The Haar measure $\mu_{\mathrm{N} \rtimes_{\varphi} \mathrm{H}}$ on $\mathrm{N} \rtimes_{\varphi} \mathrm{H}$ is then given by

$$
\int_{\mathrm{N} \rtimes_{\varphi} \mathrm{H}} f(n, h) \mathrm{d} \mu_{\mathrm{N} \rtimes_{\varphi} \mathrm{H}}(n, h):=\int_{\mathrm{H}} \int_{\mathrm{N}} f(n, h) \sigma_{\mathrm{H}}(h)^{-1} \mathrm{~d} \mu_{\mathrm{N}}(n) \mathrm{d} \mu_{\mathrm{H}}(h) .
$$

This is trivially invariant under the left $\mathrm{N}$-action, and it is also invariant under the left $\mathrm{H}$-action $(n, h) \mapsto\left(1, h^{\prime}\right)(n, h)=\left({ }^{h^{\prime}} n, h^{\prime} h\right)$, using $(2.5)$ with $F\left({ }^{\prime} n\right):=f\left({ }^{\prime} n, h^{\prime} h\right)$ and recalling that $h$ is fixed in $(2.5)$.

Theorem 2.5. Let $\mathrm{N}$ and $\mathrm{H}$ be locally compact groups and $\varphi: \mathrm{H} \rightarrow \operatorname{Aut}(\mathrm{N})$ a continuous action of $\mathrm{H}$ on $\mathrm{N}$. Define $\beta: \mathrm{H} \rightarrow \operatorname{Aut}\left(C^{*}(\mathrm{~N})\right)$ by $\left(\beta_{h^{\prime}} \ell\right)(n)=\sigma_{\mathrm{H}}\left(h^{\prime}\right)^{-1} \ell\left({h^{\prime-1}}_{n}\right)$ for all $h^{\prime} \in \mathrm{H}$ and $\ell \in C_{\mathrm{c}}(\mathrm{N})$. Then

$$
C^{*}(\mathrm{~N}) \rtimes_{\beta} \mathrm{H} \simeq C^{*}\left(\mathrm{~N} \rtimes_{\varphi} \mathrm{H}\right) .
$$

For a full proof of Theorem 2.5 that takes into account the topological and $C^{*}$-algebraic aspects, see [52, Proposition 3.11]. Here we shall just show that under the canonical injection $C_{\mathrm{c}}\left(\mathrm{N} \rtimes_{\varphi} \mathrm{H}\right) \hookrightarrow C_{\mathrm{c}}(\mathrm{N}) \rtimes_{\beta} \mathrm{H}$, given by $f(n, h) \mapsto f(h)$ where $f(h)(n)=f(n, h)$, the convolution product in $C_{\mathrm{c}}\left(\mathrm{N} \rtimes_{\varphi} \mathrm{H}\right)$ is mapped to the convolution product in $C_{\mathrm{c}}(\mathrm{N}) \rtimes_{\beta} \mathrm{H}$. Let $f, f^{\prime} \in$ $C_{\mathrm{c}}\left(\mathrm{N} \rtimes_{\varphi} \mathrm{H}\right)$, then

$$
\left(f \star_{C_{\mathrm{c}}\left(\mathrm{N} \rtimes_{\varphi} \mathrm{H}\right)} f^{\prime}\right)(n, h)=\int_{\mathrm{H}} \int_{\mathrm{N}} f\left(n^{\prime}, h^{\prime}\right) f^{\prime}\left(\left(n^{\prime}, h^{\prime}\right)^{-1}(n, h)\right) \sigma_{\mathrm{H}}\left(h^{\prime}\right)^{-1} \mathrm{~d} \mu_{\mathrm{N}}\left(n^{\prime}\right) \mathrm{d} \mu_{\mathrm{H}}\left(h^{\prime}\right) .
$$

On the other hand, for the images of $f, f^{\prime}$ in $C_{c}(\mathrm{~N}) \rtimes_{\beta} \mathrm{H}$ we have

$$
\left(f \star_{C_{\mathrm{c}}(\mathrm{N}) \rtimes_{\beta} \mathrm{H}} f^{\prime}\right)(h)=\int_{\mathrm{H}} f\left(h^{\prime}\right) \star_{C^{*}(\mathrm{~N})} \beta_{h^{\prime}}\left(f^{\prime}\left(h^{\prime-1} h\right)\right) \mathrm{d} \mu_{\mathrm{H}}\left(h^{\prime}\right) .
$$

Using the convolution product in $C^{*}(\mathrm{~N})$ this can be written as

$$
\left(f \star_{C_{\mathrm{c}}(\mathrm{N}) \rtimes_{\beta} \mathrm{H}} f^{\prime}\right)(h)(n)=\int_{\mathrm{H}} \int_{\mathrm{N}} f\left(h^{\prime}\right)\left(n^{\prime}\right) \beta_{h^{\prime}}\left(f^{\prime}\left(h^{\prime-1} h\right)\right)\left(n^{\prime-1} n\right) \mathrm{d} \mu_{\mathrm{N}}\left(n^{\prime}\right) \mathrm{d} \mu_{\mathrm{H}}\left(h^{\prime}\right),
$$

which from the definition of the action $\beta$ is easily seen to equal the image $\left(f \star_{C_{\mathrm{c}}}\left(\mathrm{N} \rtimes_{\varphi} \mathrm{H}\right) f^{\prime}\right)(h)(n)$ in $C_{\mathrm{c}}(\mathrm{N}) \rtimes_{\beta} \mathrm{H}$ of the product $\left(f \star_{C_{\mathrm{c}}}\left(\mathrm{N} \rtimes_{\varphi} \mathrm{H}\right) f^{\prime}\right)(n, h)$ in $C_{\mathrm{c}}\left(\mathrm{N} \rtimes_{\varphi} \mathrm{H}\right)$ from $(2.6)$.

More generally we have [52, Proposition 3.11] 
Theorem 2.6. Let $\left(\mathcal{A}, \mathrm{N} \rtimes_{\varphi} \mathrm{H}, \alpha\right)$ be a $C^{*}$-dynamical system for the semi-direct product group $\mathrm{N} \rtimes_{\varphi} \mathrm{H}$. Then $\left(\mathcal{A} \rtimes_{\left.\alpha\right|_{\mathrm{N}}} \mathrm{N}, \mathrm{H}, \beta\right)$ is a $C^{*}$-dynamical system, where

$$
\beta: \mathrm{H} \longrightarrow \operatorname{Aut}\left(\mathcal{A} \rtimes_{\left.\alpha\right|_{\mathrm{N}}} \mathrm{N}\right), \quad h \longmapsto \beta_{h}
$$

is defined by $\left(\beta_{h}(f)\right)(n)=\sigma_{\mathrm{H}}(h)^{-1} \alpha_{h}\left(f\left({ }^{h^{-1}} n\right)\right)$ for all $f \in C_{\mathrm{c}}(\mathrm{N}, \mathcal{A}) \subset \mathcal{A} \rtimes_{\left.\alpha\right|_{\mathrm{N}}} \mathrm{N}$, with $\sigma_{\mathrm{H}}: \mathrm{H} \rightarrow$ $\mathbb{R}^{+}$defined by (2.5) and ${ }^{h^{-1}} n=\varphi_{h^{-1}}(n)$. Moreover, the canonical injection $C_{\mathrm{c}}\left(\mathrm{N} \rtimes_{\varphi} \mathrm{H}, \mathcal{A}\right) \hookrightarrow$ $C_{\mathrm{c}}\left(\mathrm{H}, C_{\mathrm{c}}(\mathrm{N}, \mathcal{A})\right)$ extends to a $C^{*}$-algebra isomorphism

$$
\mathcal{A} \rtimes_{\alpha}\left(\mathrm{N} \rtimes_{\varphi} \mathrm{H}\right) \simeq\left(\mathcal{A} \rtimes_{\left.\alpha\right|_{\mathrm{N}}} \mathrm{N}\right) \rtimes_{\beta} \mathrm{H} .
$$

Theorem 2.5 is then recovered by setting $\mathcal{A}=\mathbb{C}$.

In the spirit of Theorem 2.5, which shows that crossed products are a generalization of semi-direct products, let us mention the semi-direct product construction behind Theorem 2.6. Consider three groups $\mathrm{M}, \mathrm{N}$ and $\mathrm{H}$ with group actions ${ }^{\mathrm{H}} \mathrm{N}$ and ${ }^{\mathrm{N} \rtimes \mathrm{H}} \mathrm{M}$; then there are also group actions ${ }^{\mathrm{N}} \mathrm{M}$ and ${ }^{\mathrm{H}} \mathrm{M}$. The associativity of the triple semi-direct product construction is then easily established through

Proposition 2.7. Let $\mathrm{M}, \mathrm{N}$ and $\mathrm{H}$ be groups with group actions ${ }^{\mathrm{H}} \mathrm{M},{ }^{\mathrm{H}} \mathrm{N}$ and ${ }^{\mathrm{N}} \mathrm{M}$ satisfying the compatibility conditions

$$
{ }^{h}\left({ }^{n} m\right)=\left({ }^{h} n\right)\left({ }^{h} m\right)
$$

for all $m \in \mathrm{M}, n \in \mathrm{N}$ and $h \in \mathrm{H}$. Then there exists a group action ${ }^{\mathrm{N} \rtimes \mathrm{H}} \mathrm{M}$ defined by ${ }^{(n, h)} m=$ ${ }^{n}\left({ }^{h} m\right)$, and a group action ${ }^{\mathrm{H}}(\mathrm{M} \rtimes \mathrm{N})$ defined by ${ }^{h}(m, n)=\left({ }^{h} m,{ }^{h} n\right)$, which together satisfy the associativity property

$$
\mathrm{M} \rtimes(\mathrm{N} \rtimes \mathrm{H})=(\mathrm{M} \rtimes \mathrm{N}) \rtimes \mathrm{H} .
$$

\subsection{Pontryagin duality and Fourier transform}

If $\mathrm{N}$ is a locally compact abelian group we denote by $\widehat{N}$ its Pontryagin dual, i.e., the set of characters $\chi: \mathrm{N} \rightarrow \mathrm{U}(1)$, which is also a locally compact abelian group (with the compact-open topology and with the pointwise multiplication). For example, if $N=\mathbb{R}^{d}$ then $\widehat{N}=\mathbb{R}^{d}$ and the characters are given by $\chi_{p}(x)=\mathrm{e}^{2 \pi \mathrm{i}\langle p, x\rangle}$ for $x_{\hat{\mathrm{N}}} \in \mathrm{N}$ and $p \in \widehat{\mathrm{N}}$. The Pontryagin duality theorem states that there is a canonical isomorphism $\widehat{\mathrm{N}} \simeq \mathrm{N}$, where $n \in \mathrm{N}$ is associated to the character $\chi \mapsto \chi(n)$ on $\widehat{\mathrm{N}}$.

The Fourier transform shows that the group $C^{*}$-algebra $C^{*}(\mathrm{~N})$ is isomorphic to $C_{0}(\widehat{\mathrm{N}})$ : Given a Haar measure $\mu_{\mathrm{N}}$ on $\mathrm{N}$, the Fourier transform $\mathcal{F}(f)$ of $f \in C_{\mathrm{c}}(\mathrm{N})$ is defined by

$$
\mathcal{F}(f)(\chi):=\int_{\mathrm{N}} f(n) \chi(n) \mathrm{d} \mu_{\mathrm{N}}(n)
$$

for $\chi \in \widehat{\mathrm{N}}$. It sends the convolution product of functions in $C^{*}(\mathrm{~N})$ to the pointwise product of functions in $C(\widehat{\mathrm{N}})$ :

$$
\mathcal{F}\left(f \star f^{\prime}\right)=\mathcal{F}(f) \mathcal{F}\left(f^{\prime}\right),
$$

and extends to an isomorphism [52, Proposition 3.1]

$$
\mathcal{F}: C^{*}(\mathrm{~N}) \stackrel{\simeq}{\longrightarrow} C_{0}(\widehat{\mathrm{N}}),
$$

where $C_{0}(\widehat{\mathrm{N}})$ is the algebra of functions on $\widehat{\mathrm{N}}$ vanishing at infinity. For $\mathrm{N}$ separable, Hausdorff and locally compact, $C_{0}(\widehat{\mathrm{N}})$ is indeed a $C^{*}$-algebra. 
Given a continuous left group action $\varphi: \mathrm{H} \rightarrow \operatorname{Aut}(\mathrm{N})$, which we also denote as before by $\varphi_{h}(n)={ }^{h} n$, consider the induced action $\beta: \mathrm{H} \rightarrow \operatorname{Aut}\left(C^{*}(\mathrm{~N})\right)$ as defined in Theorem 2.5. There is also an induced left action $\widehat{\varphi}: H \rightarrow$ Aut $(\widehat{N})$ defined by pullback: $\left(\widehat{\varphi}_{h} \chi\right)(n):=\chi\left({ }^{h^{-1}} n\right)$, together with its pullback $\widehat{\varphi}^{*}: \mathrm{H} \rightarrow \operatorname{Aut}\left(C_{0}(\widehat{\mathrm{N}})\right)$ defined by $\left(\widehat{\varphi}_{h}^{*} \widehat{f}\right)(\chi)=\widehat{f}\left(\widehat{\varphi}_{h^{-1}} \chi\right)$ for all $h \in \mathrm{H}, \widehat{f} \in C_{0}(\widehat{\mathrm{N}})$ and $\chi \in \widehat{\mathrm{N}}$. The Fourier transform isomorphism (2.7) then extends to the isomorphism

Proposition 2.8. If $\mathrm{N}$ is a locally compact abelian group and $\varphi: \mathrm{H} \rightarrow \operatorname{Aut}(\mathrm{N})$ is a continuous action of a locally compact group $\mathrm{H}$ on $\mathrm{N}$, then

$$
C^{*}(\mathrm{~N}) \rtimes_{\beta} \mathrm{H} \simeq C_{0}(\widehat{\mathrm{N}}) \rtimes_{\widehat{\varphi}^{*}} \mathrm{H} .
$$

Proof. We show that the triples $\left(C^{*}(\mathrm{~N}), \mathrm{H}, \beta\right)$ and $\left(C_{0}(\widehat{\mathrm{N}}), \mathrm{H}, \widehat{\varphi}^{*}\right)$ are equivalent dynamical systems, see [52, Example 3.16]. For this, we prove that the Fourier transform (2.7) is $\mathrm{H}$ equivariant with respect to the $\mathrm{H}$-actions $\beta$ and $\widehat{\varphi}^{*}$. For $h \in \mathrm{H}, f \in C_{\mathrm{c}}(\mathrm{N})$ and $\chi \in \widehat{\mathrm{N}}$ we compute

$$
\begin{aligned}
\mathcal{F}\left(\beta_{h}(f)\right)(\chi) & =\int_{\mathrm{N}} \beta_{h}(f)(n) \chi(n) \mathrm{d} \mu_{\mathrm{N}}(n)=\sigma_{\mathrm{H}}(h)^{-1} \int_{\mathrm{N}} f\left({ }^{-1} n\right) \chi(n) \mathrm{d} \mu_{\mathrm{N}}(n) \\
& =\sigma_{\mathrm{H}}(h)^{-1} \int_{\mathrm{N}} f\left({ }^{h^{-1}} n\right) \chi\left({ }^{h}\left({ }^{-1} n\right)\right) \mathrm{d} \mu_{\mathrm{N}}(n)=\int_{\mathrm{N}} f(n) \chi\left({ }^{h} n\right) \mathrm{d} \mu_{\mathrm{N}}(n) \\
& =\mathcal{F}(f)\left(\widehat{\varphi}_{h^{-1}} \chi\right)=\left(\widehat{\varphi}_{h}^{*} \mathcal{F}(f)\right)(\chi),
\end{aligned}
$$

where in the fourth equality we used (2.5) with $F\left({ }^{-1} n\right)=f\left({ }^{-1} n\right) \chi\left({ }^{h}\left(h^{-1} n\right)\right)$.

Replacing $\mathrm{N}$ with $\widehat{\mathrm{N}}$ in Proposition 2.8 we also obtain the isomorphism

$$
C^{*}(\widehat{\mathrm{N}}) \rtimes_{\widehat{\beta}} \mathrm{H} \simeq C_{0}(\widehat{\widehat{N}}) \rtimes_{\widehat{\varphi}^{*}} \mathrm{H} \simeq C_{0}(\mathrm{~N}) \rtimes_{\varphi^{*}} \mathrm{H},
$$

where $\widehat{\beta}: \mathbf{H} \rightarrow \operatorname{Aut}\left(C^{*}(\widehat{\mathrm{N}})\right)$ is defined by $\widehat{\beta}_{h}(\widehat{f})(\chi)=\widehat{\sigma}_{\mathbf{H}}(h)^{-1} \widehat{f}\left(\widehat{\varphi}_{h^{-1}} \chi\right)$ for $h \in \mathbf{H}, \widehat{f} \in C_{\mathrm{c}}(\widehat{\mathrm{N}})$ and $\chi \in \widehat{\mathrm{N}}$, with $\widehat{\sigma}_{\mathrm{H}}: \mathrm{H} \rightarrow \mathbb{R}^{+}$defined as in (2.5) but using the dual group $\widehat{\mathrm{N}}$ instead of $\mathrm{N}$, and in the final isomorphism we used Pontryagin duality $\widehat{\mathrm{N}} \simeq \mathrm{N}$.

Another important property of crossed products is Takai duality [52, Section 7.1]. If $\mathrm{G}$ is a locally compact abelian group and $(\mathcal{A}, \mathrm{G}, \alpha)$ is a $C^{*}$-dynamical system, then $\left(\mathcal{A} \rtimes_{\alpha} \mathrm{G}, \widehat{\mathrm{G}}, \widehat{\alpha}\right)$ is a $C^{*}$-dynamical system, where

$$
\widehat{\alpha}: \widehat{\mathrm{G}} \longrightarrow \operatorname{Aut}\left(\mathcal{A} \rtimes_{\alpha} \mathrm{G}\right), \quad \chi \longmapsto \widehat{\alpha}_{\chi}
$$

is defined by $\widehat{\alpha}_{\chi}(f)(\gamma):=\overline{\chi(\gamma)} f(\gamma)$ for all $f \in C_{\mathrm{c}}(\mathrm{G}, \mathcal{A})$.

Theorem 2.9 (Takai duality). Let $(\mathcal{A}, \mathrm{G}, \alpha)$ be a $C^{*}$-dynamical system where $\mathrm{G}$ is a locally compact abelian group. Then there is an isomorphism of $C^{*}$-algebras

$$
\left(\mathcal{A} \rtimes_{\alpha} \mathrm{G}\right) \rtimes_{\widehat{\alpha}} \widehat{\mathrm{G}} \simeq \mathcal{A} \otimes \mathcal{K}\left(\mathrm{L}^{2}(\mathrm{G})\right) .
$$

\subsection{Morita equivalence and Green's theorem}

Crossed products of algebras provide a host of examples of dualities which come in the form of various levels of strong and weak equivalences of algebras, see, e.g., [6]. The most primitive form of such dualities is provided by (strong) Morita equivalence [41]. A bimodule for a pair of algebras $\mathcal{A}$ and $\mathcal{B}$ is a vector space $\mathcal{M}$ which is simultaneously a left $\mathcal{A}$-module and a right $\mathcal{B}$-module, where the left action of $\mathcal{A}$ commutes with the right action of $\mathcal{B}:(a \cdot \xi) \cdot b=a \cdot(\xi \cdot b)$ 
for all $a \in \mathcal{A}, b \in \mathcal{B}$ and $\xi \in \mathcal{M}$. If $\mathcal{A}$ and $\mathcal{B}$ are $C^{*}$-algebras, we say that a bimodule $\mathcal{M}$ is an $\mathcal{A}-\mathcal{B}$ Morita equivalence bimodule (or imprimitivity bimodule) if it is equipped with an $\mathcal{A}$-valued inner product $\mathcal{A}\langle\cdot, \cdot\rangle$ and a $\mathcal{B}$-valued inner product $\langle\cdot, \cdot\rangle_{\mathcal{B}}$ satisfying the associativity condition

$$
\mathcal{A}_{\mathcal{A}}\langle\psi, \phi\rangle \cdot \xi=\psi \cdot\langle\phi, \xi\rangle_{\mathcal{B}},
$$

for all $\psi, \phi, \xi \in \mathcal{M}$, under which $\mathcal{M}$ is complete in the norm closures, and such that the ideal ${ }_{\mathcal{A}}\langle\mathcal{M}, \mathcal{M}\rangle$ is dense in $\mathcal{A}$ and $\langle\mathcal{M}, \mathcal{M}\rangle_{\mathcal{B}}$ is dense in $\mathcal{B}$. The bimodule $\mathcal{M}$ establishes a Morita equivalence between the algebras $\mathcal{A}$ and $\mathcal{B}$, and in this case we write $\mathcal{A} \sim_{\mathrm{M}} \mathcal{B}$.

Morita equivalent $C^{*}$-algebras have equivalent categories of nondegenerate $*$-representations: If $\Pi_{\mathcal{B}}: \mathcal{B} \rightarrow \mathrm{B}\left(\mathcal{H}_{\mathcal{B}}\right)$ is a representation of $\mathcal{B}$ on a Hilbert space $\mathcal{H}_{\mathcal{B}}$, then we can construct another Hilbert space

$$
\mathcal{H}_{\mathcal{A}}:=\mathcal{M} \otimes_{\mathcal{B}} \mathcal{H}_{\mathcal{B}}
$$

which is the quotient of the tensor product $\mathcal{M} \otimes \mathcal{H}_{\mathcal{B}}$ by the relation $(\xi \cdot b) \otimes \psi-\xi \otimes \Pi_{\mathcal{B}}(b) \psi=0$ identifying the $\mathcal{B}$-actions for $\xi \in \mathcal{M}, b \in \mathcal{B}$ and $\psi \in \mathcal{H}_{\mathcal{B}}$. The inner product on $\mathcal{H}_{\mathcal{A}}$ is given by

$$
\left\langle\xi \otimes_{\mathcal{B}} \psi \mid \xi^{\prime} \otimes_{\mathcal{B}} \psi^{\prime}\right\rangle_{\mathcal{H}_{\mathcal{A}}}:=\left\langle\psi \mid \Pi_{\mathcal{B}}\left(\left\langle\xi, \xi^{\prime}\right\rangle_{\mathcal{B}}\right) \psi^{\prime}\right\rangle_{\mathcal{H}_{\mathcal{B}}}
$$

and a representation $\Pi_{\mathcal{A}}: \mathcal{A} \rightarrow \mathrm{B}\left(\mathcal{H}_{\mathcal{A}}\right)$ of the algebra $\mathcal{A}$ is defined by

$$
\Pi_{\mathcal{A}}(a)\left(\xi \otimes_{\mathcal{B}} \psi\right)=(a \cdot \xi) \otimes_{\mathcal{B}} \psi
$$

for $a \in \mathcal{A}$ and $\xi \otimes_{\mathcal{B}} \psi \in \mathcal{H}_{\mathcal{A}}$; this representation is unitary equivalent to the representation $\Pi_{\mathcal{B}}$. Conversely, starting with a representation of $\mathcal{A}$, we can use a conjugate $\mathcal{B}-\mathcal{A}$ equivalence bimodule $\overline{\mathcal{M}}$ to construct a unitary equivalent representation of $\mathcal{B}$; then there are surjective bimodule homomorphisms $\mathcal{M} \otimes_{\mathcal{B}} \overline{\mathcal{M}} \rightarrow \mathcal{A}$ and $\overline{\mathcal{M}} \otimes_{\mathcal{A}} \mathcal{M} \rightarrow \mathcal{B}$ which satisfy a certain transitivity law. As a particular consequence of this equivalence, Morita equivalent algebras have homeomorphic spectra and isomorphic K-theory groups.

Example 2.10 (noncommutative two-tori). A famous example of Morita equivalence in both mathematics and string theory is provided by the noncommutative tori $A_{\theta}=\mathbb{T}_{\theta}^{2}$ from Example 2.1. Firstly, notice from (2.3) that changing the coset representative $\theta \in \mathbb{R} / \mathbb{Z}$ yields an identical algebra: $\mathrm{A}_{\theta+m}=\mathrm{A}_{\theta}$ for all $m \in \mathbb{Z}$. Secondly, there is an obvious $C^{*}$-algebra isomorphism $\mathrm{A}_{-\theta} \simeq \mathrm{A}_{\theta}$ obtained by interchanging the two generators $U$ and $V$. The converse is also true [43, 44]: $A_{\theta^{\prime}} \simeq \mathrm{A}_{\theta}$ if and only if $\theta^{\prime}=\theta \bmod 1$. More generally, two irrational rotation $C^{*}$-algebras $\mathrm{A}_{\theta}$ and $\mathrm{A}_{\theta^{\prime}}$ are Morita equivalent if and only if $\theta$ and $\theta^{\prime}$ lie in the same orbit under the action of $\mathrm{GL}(2, \mathbb{Z})$ by fractional linear transformations

$$
\theta^{\prime}=\mathrm{M}[\theta]:=\frac{a \theta+b}{c \theta+d} \quad \text { for } \quad \mathrm{M}=\left(\begin{array}{ll}
a & b \\
c & d
\end{array}\right) \in \mathrm{GL}(2, \mathbb{Z}) .
$$

The explicit Morita equivalence bimodules can be found in [43]. On the other hand, the rational rotation algebras $\mathrm{A}_{\theta}$ are all Morita equivalent to the commutative algebra $C\left(\mathbb{T}^{2}\right)$ of functions on the two-torus [44].

Example 2.11 (noncommutative $d$-tori). The Morita equivalences of Example 2.10 generalize to the higher-dimensional noncommutative tori $A_{\Theta}=\mathbb{T}_{\Theta}^{d}$ from Example 2.2 in the following way [46]. First of all, the algebra $A_{\Theta}$ is unchanged if the matrix $\Theta$ is written in another basis of $\mathbb{Z}^{d}$ : if $B \in \mathrm{GL}(d, \mathbb{Z})$ with transpose $B^{\mathrm{t}}$, then there is a $C^{*}$-algebra isomorphism $A_{B^{\mathrm{t}}} \Theta B \simeq \mathrm{A}_{\Theta}$. More generally, consider the set of real skew-symmetric $d \times d$ matrices $\Theta$ whose orbits $\boldsymbol{M}[\Theta]$ are defined for all $\boldsymbol{M} \in \mathrm{SO}(d, d ; \mathbb{Z})$, where

$$
\boldsymbol{M}[\Theta]=(A \Theta+B)(C \Theta+D)^{-1} \quad \text { for } \quad \boldsymbol{M}=\left(\begin{array}{ll}
A & B \\
C & D
\end{array}\right) \in \mathrm{SO}(d, d ; \mathbb{Z}),
$$


and $A, B, C$ and $D$ are $d \times d$ block matrices satisfying

$$
A^{\mathrm{t}} C+C^{\mathrm{t}} A=0=B^{\mathrm{t}} D+D^{\mathrm{t}} B \quad \text { and } \quad A^{\mathrm{t}} D+C^{\mathrm{t}} B=\mathbb{1}_{d} .
$$

The set of all such matrices is dense in the space of all skew-symmetric real $d \times d$ matrices, and there is a Morita equivalence

$$
\mathrm{A}_{M[\Theta]} \sim \mathrm{A}_{\Theta}
$$

However, for $d>2$ the converse is not generally true: In fact, there are algebras $A_{\Theta}$ and $A_{\Theta^{\prime}}$ that are isomorphic (and so Morita equivalent) but for which the matrices $\Theta$ and $\Theta^{\prime}$ do not belong to the same $\mathrm{SO}(d, d ; \mathbb{Z})$ orbit [46].

We will also need an equivariant version of Morita equivalence in order to show that Morita equivalent algebras induce Morita equivalent crossed products according to [14, Section 5.4]

Theorem 2.12. Let $(\mathcal{A}, \mathrm{G}, \alpha)$ and $(\mathcal{B}, \mathrm{G}, \beta)$ be $C^{*}$-dynamical systems such that $\mathcal{A}$ and $\mathcal{B}$ are Morita equivalent. Then the crossed product $C^{*}$-algebras $\mathcal{A} \rtimes_{\alpha} \mathrm{G}$ and $\mathcal{B} \rtimes_{\beta} \mathrm{G}$ are Morita equivalent if there exists a $\mathrm{G}$-equivariant $\mathcal{A}-\mathcal{B}$ Morita equivalence bimodule $\mathcal{M}$, i.e., if there is a strongly continuous action $U: \mathrm{G} \rightarrow \operatorname{Aut}(\mathcal{M})$ of $\mathrm{G}$ on an $\mathcal{A}-\mathcal{B}$ Morita equivalence bimodule $\mathcal{M}$ such that

$$
U_{\gamma}(a \cdot \xi)=\alpha_{\gamma}(a) \cdot U_{\gamma}(\xi) \quad \text { and } \quad U_{\gamma}(\xi \cdot b)=U_{\gamma}(\xi) \cdot \beta_{\gamma}(b),
$$

and

$$
{ }_{\mathcal{A}}\left\langle U_{\gamma}(\xi), U_{\gamma}\left(\xi^{\prime}\right)\right\rangle=\alpha_{\gamma}\left({ }_{\mathcal{A}}\left\langle\xi, \xi^{\prime}\right\rangle\right) \quad \text { and } \quad\left\langle U_{\gamma}(\xi), U_{\gamma}\left(\xi^{\prime}\right)\right\rangle_{\mathcal{B}}=\beta_{\gamma}\left(\left\langle\xi, \xi^{\prime}\right\rangle_{\mathcal{B}}\right),
$$

for all $\gamma \in \mathrm{G}, \xi, \xi^{\prime} \in \mathcal{M}, a \in \mathcal{A}$ and $b \in \mathcal{B}$.

In this paper, our main application of Morita equivalence will involve Green's symmetric imprimitivity theorem. Let $X$ be a locally compact space, and let $\mathrm{H}$ and $\mathrm{K}$ be locally compact groups with commuting free and proper actions on the right and on the left on $X$, respectively. We can lift these actions to left actions on $C_{0}(X)$ by defining $\left({ }^{h} \mathbf{f}\right)(x)=\mathbf{f}\left(h^{-1} \cdot x\right)$ and $\left({ }^{k} \mathbf{f}\right)(x)=$ $\mathrm{f}(x \cdot k)$ for all $\mathrm{f} \in C_{0}(X), x \in X, h \in \mathrm{H}$ and $k \in \mathrm{K}$. Commutativity of the actions of $\mathrm{H}$ and $\mathrm{K}$ implies that there are well-defined induced actions of $\mathrm{H}$ and $\mathrm{K}$ respectively on the quotient spaces $\mathrm{K} \backslash X$ and $X / \mathrm{H}$, and hence respectively on the algebras $C_{0}(\mathrm{~K} \backslash X)$ and $C_{0}(X / \mathrm{H})$ which we denote rt and lt. Green's symmetric imprimitivity theorem then reads as [52, Corollary 4.10]

Theorem 2.13. There is a Morita equivalence of $C^{*}$-algebras

$$
C_{0}(\mathrm{~K} \backslash X) \rtimes_{\mathrm{rt}} \mathrm{H} \sim_{\mathrm{M}} C_{0}(X / \mathrm{H}) \rtimes_{\mathrm{lt}} \mathrm{K}
$$

implemented by the Morita equivalence (or imprimitivity) bimodule $\mathcal{M}$ which is the completion of $C_{\mathrm{c}}(X)$ with the actions

$$
\begin{aligned}
& (a \cdot \xi)(x)=\int_{\mathrm{K}} a(k, x \cdot \mathrm{H}) \xi\left(k^{-1} \cdot x\right) \Delta_{\mathrm{K}}(k)^{1 / 2} \mathrm{~d} \mu_{\mathrm{K}}(k), \\
& (\xi \cdot b)(x)=\int_{\mathbf{H}} \xi\left(x \cdot h^{-1}\right) b\left(h, \mathrm{~K} \cdot x \cdot h^{-1}\right) \Delta_{\mathrm{H}}(h)^{-1 / 2} \mathrm{~d} \mu_{\mathrm{H}}(h),
\end{aligned}
$$

for all $x \in X, a \in C_{\mathrm{c}}(\mathrm{K} \times X / \mathrm{H}), b \in C_{\mathrm{c}}(\mathrm{H} \times \mathrm{K} \backslash X)$ and $\xi \in C_{\mathrm{c}}(X)$, and the inner products

$$
\begin{aligned}
& \mathcal{A}\left\langle\xi, \xi^{\prime}\right\rangle(k, x \cdot \mathrm{H})=\Delta_{\mathrm{K}}(k)^{-1 / 2} \int_{\mathrm{H}} \xi(x \cdot h) \overline{\xi^{\prime}\left(k^{-1} \cdot x \cdot h\right)} \mathrm{d} \mu_{\mathrm{H}}(h), \\
& \left\langle\xi, \xi^{\prime}\right\rangle_{\mathcal{B}}(h, \mathrm{~K} \cdot x)=\Delta_{\mathrm{H}}(h)^{-1 / 2} \int_{\mathbf{K}} \overline{\xi\left(k^{-1} \cdot x\right)} \xi^{\prime}\left(k^{-1} \cdot x \cdot h\right) \mathrm{d} \mu_{\mathrm{K}}(k),
\end{aligned}
$$

for all $x \in X, h \in \mathrm{H}, k \in \mathrm{K}$ and $\xi, \xi^{\prime} \in C_{\mathrm{c}}(X)$. 
Theorem 2.13 has several useful applications and corollaries, see, e.g., [42]. A particularly relevant special case that we shall use below is when $\mathrm{K}$ is the trivial group, in which case (2.9) reduces to the Morita equivalence

$$
C_{0}(X) \rtimes_{\mathrm{rt}} \mathrm{H} \sim_{\mathrm{M}} C_{0}(X / \mathrm{H})
$$

illustrating the use of crossed products in describing quotients. This equivalence can in fact be strengthened to a stable isomorphism [42]

$$
C_{0}(X) \rtimes_{\mathrm{rt}} \mathrm{H} \simeq C_{0}(X / \mathrm{H}) \otimes \mathcal{K}\left(\mathrm{L}^{2}(\mathrm{H})\right),
$$

where $\mathcal{K}$ denotes the algebra of compact operators.

Example 2.14 (tori). A particularly relevant example for us is the case $X=\mathbb{R}^{d}$ with $\mathrm{H}=\mathbb{Z}^{d}$ acting by translations $(n, x) \mapsto x+n$ for $n \in \mathbb{Z}^{d}$ and $x \in \mathbb{R}^{d}$, which realizes the $d$-dimensional torus $\mathbb{T}^{d}=\mathbb{R}^{d} / \mathbb{Z}^{d}$ as a crossed product:

$$
C\left(\mathbb{T}^{d}\right) \sim_{\mathrm{M}} C_{0}\left(\mathbb{R}^{d}\right) \rtimes_{\mathrm{rt}} \mathbb{Z}^{d}
$$

Let us illustrate the construction explicitly. The convolution algebra $C_{\mathrm{c}}\left(\mathbb{Z}^{d} \times \mathbb{R}^{d}\right) \subset C_{0}\left(\mathbb{R}^{d}\right) \rtimes_{\mathrm{rt}} \mathbb{Z}^{d}$ can be identified with the space of sequences $f=\left\{f_{n}\right\}_{n \in \mathbb{Z}^{d}}$ of functions $f_{n}: \mathbb{R}^{d} \rightarrow \mathbb{C}$ with the convolution product

$$
(f \star g)_{n}(x)=\sum_{m \in \mathbb{Z}^{d}} f_{m}(x) g_{n-m}(x-m) .
$$

Consider the algebra

$$
\mathcal{A}:=\left\{f \in C_{0}\left(\mathbb{R}^{d}, \mathcal{K}\left(\ell^{2}\left(\mathbb{Z}^{d}\right)\right)\right) \mid f(x+m)=U_{m} f(x) U_{m}^{-1}\right\},
$$

where $U_{m}$ is the unitary shift operator on $\ell^{2}\left(\mathbb{Z}^{d}\right)$ defined by $\left(U_{m} a\right)_{n}=a_{n-m}$ for each $m \in \mathbb{Z}^{d}$ and $a=\left\{a_{n}\right\}_{n \in \mathbb{Z}^{d}}$. Define a map $\Phi: C_{\mathrm{c}}\left(\mathbb{Z}^{d} \times \mathbb{R}^{d}\right) \rightarrow C_{\mathrm{c}}\left(\mathbb{R}^{d}, \mathcal{K}\left(\ell^{2}\left(\mathbb{Z}^{d}\right)\right)\right)$ by

$$
(\Phi(f)(x))_{m n}=f_{m+n}(x+n) .
$$

It is easy to see that $\Phi(f)(x+m)=U_{m} \Phi(f)(x) U_{m}^{-1}$ for all $f \in C_{\mathrm{c}}\left(\mathbb{Z}^{d} \times \mathbb{R}^{d}\right)$, and if $a=\left(a_{m n}\right) \in \mathcal{A}$ then defining $f_{n}:=a_{0 n}$ gives $\Phi(f)=a$. It is also easy to check that $\Phi(f \star g)=\Phi(f) \Phi(g)$, and consequently $\Phi$ gives an algebra isomorphism

$$
\Phi: C_{0}\left(\mathbb{R}^{d}\right) \rtimes_{\mathrm{rt}} \mathbb{Z}^{d} \stackrel{\simeq}{\longrightarrow} \mathcal{A} .
$$

The explicit Morita equivalence bimodule is now obtained from the completion of

$$
\mathcal{M}=\left\{\xi \in C_{\mathrm{c}}\left(\mathbb{R}^{d}, \ell^{2}\left(\mathbb{Z}^{d}\right)\right) \mid \xi(x+m)=U_{m} \xi(x)\right\} .
$$

The left action of the algebra $\mathcal{A}=\Phi\left(C_{\mathrm{c}}\left(\mathbb{Z}^{d} \times \mathbb{R}^{d}\right)\right)$ is by left matrix multiplication on $\mathcal{M}$ :

$$
\begin{aligned}
\mathcal{A} \times \mathcal{M} & \longrightarrow \mathcal{M}, \\
(a, \xi) & \longmapsto a \cdot \xi, \quad(a \cdot \xi)_{n}=\sum_{m \in \mathbb{Z}^{d}} a_{n m} \xi_{m},
\end{aligned}
$$

while the right action of the algebra $C\left(\mathbb{T}^{d}\right)$ is by right pointwise multiplication on $\mathcal{M}$ :

$$
\begin{aligned}
\mathcal{M} \times C\left(\mathbb{T}^{d}\right) & \longrightarrow \mathcal{M}, \\
(\xi, b) & \longmapsto \xi \cdot b, \quad(\xi \cdot b)_{n}=\xi_{n} b .
\end{aligned}
$$


The left and right inner products are respectively given by

$$
\begin{aligned}
& \mathcal{A}\langle\xi, \eta\rangle=\xi \otimes \eta^{*}, \\
& \langle\xi, \eta\rangle_{C\left(\mathbb{T}^{d}\right)}=\sum_{n \in \mathbb{Z}^{d}} \overline{\xi_{n}} \eta_{n},
\end{aligned}
$$

for $\xi, \eta \in \mathcal{M}$. Together with the isomorphism $\Phi$, this establishes a Morita equivalence between the algebras $C_{0}\left(\mathbb{R}^{d}\right) \rtimes_{\mathrm{rt}} \mathbb{Z}^{d}$ and $C\left(\mathbb{T}^{d}\right)$.

Another important class of examples is provided by taking $X=\mathrm{G}$ to be a locally compact group with closed subgroups $\mathrm{K}$ and $\mathrm{H}$ acting respectively by left and right multiplication on $\mathrm{G}$. In particular, in the special case $\mathrm{K}=\mathrm{G}$, so that $C_{0}(\mathrm{~K} \backslash \mathrm{G})=\mathbb{C}$, Theorem 2.13 gives a Morita equivalence between the commutative dynamical system $\left(C_{0}(\mathrm{G} / \mathrm{H}), \mathrm{G}, \mathrm{lt}\right)$, with $\mathrm{K}=\mathrm{G}$ acting by left multiplication on the homogeneous space $\mathrm{G} / \mathrm{H}$ (so that $\left(\operatorname{lt}_{\gamma} f\right)(x)=f\left(\gamma^{-1} x\right)$ for all $f \in C_{0}(\mathrm{G} / \mathrm{H}), x \in \mathrm{G} / \mathrm{H}$ and $\left.\gamma \in \mathrm{G}\right)$, and the group $C^{*}$-algebra $C^{*}(\mathrm{H})=\mathbb{C} \rtimes \mathrm{H}$ :

$$
C_{0}(\mathrm{G} / \mathrm{H}) \rtimes_{\mathrm{lt}} \mathrm{G} \sim_{\mathrm{M}} C^{*}(\mathrm{H}) .
$$

This equivalence also follows from the $C^{*}$-algebra isomorphism [52, Theorem 4.29]

$$
C_{0}(\mathrm{G} / \mathrm{H}) \rtimes_{\mathrm{lt}} \mathrm{G} \simeq C^{*}(\mathrm{H}) \otimes \mathcal{K}\left(\mathrm{L}^{2}(\mathrm{G} / \mathrm{H})\right) .
$$

\section{Topological T-duality and twisted tori}

In this section we shall apply the results of Section 2, and in particular Green's theorem, to present a scheme that will be employed in our study of topological T-duality. We shall then illustrate how our scheme works to reproduce some standard (commutative) examples of T-dual spaces.

\subsection{Twisted tori and their T-duals}

We are interested in formulating a notion of T-duality for "torus bundles without $H$-flux", which for our purposes can be characterised by the following general class of spaces.

Definition 3.1 (twisted tori). Let $G$ be a locally compact group which admits a cocompact discrete subgroup $\Lambda_{\mathrm{G}}$, i.e., a lattice in $\mathrm{G}$, which we let act on $\mathrm{G}$ by left multiplication. The quotient space

$$
\mathbb{T}_{\Lambda_{\mathrm{G}}}:=\Lambda_{\mathrm{G}} \backslash \mathrm{G}
$$

is a twisted torus.

By [34, Lemma 6.2], only unimodular groups can contain lattices, i.e., groups $\mathrm{G}$ whose modular function $\Delta_{\mathrm{G}}$ is identically equal to 1 .

Example 3.2 (tori). Every lattice in the abelian Lie group $G=\mathbb{R}^{d}$ is isomorphic to $\Lambda_{\mathrm{G}}=\mathbb{Z}^{d}$, acting by translations. Then $\mathbb{T}_{\mathbb{Z}^{d}}=\mathbb{Z}^{d} \backslash \mathbb{R}^{d}=: \mathbb{T}^{d}$ is the $d$-dimensional torus.

Example 3.3 (nilmanifolds). Generalizing Example 3.2, let G be a connected and simplyconnected nilpotent Lie group. Then a theorem of Malcev [40, Theorem 2.12] establishes the existence of a lattice $\Lambda_{\mathrm{G}}$ in $\mathrm{G}$ if and only if $\mathrm{G}$ can be defined over the rationals, i.e., there exists a basis for its Lie algebra which has rational structure constants, and in this case $\mathbb{T}_{\Lambda_{\mathrm{G}}}=\Lambda_{\mathrm{G}} \backslash \mathrm{G}$ is a nilmanifold. 
Example 3.4 (orbifolds). Let $\mathrm{G}$ be a compact Lie group. Then the lattices in $\mathrm{G}$ are precisely the finite subgroups $\Gamma$ of $G$, and $\mathbb{T}_{\Gamma}=\Gamma \backslash G$ is a smooth orbifold. For instance, for $G=S U(2)$ the twisted tori are precisely the three-dimensional ADE orbifolds $\mathbb{T}_{\Gamma}=\Gamma \backslash \mathbb{S}^{3}$ of the three-sphere for a finite subgroup $\Gamma \subset \mathrm{SU}(2)$; for $\Gamma=\mathbb{Z}_{n}$ a cyclic subgroup of order $n \geq 2$, this recovers the familiar lens spaces $\mathbb{T}_{\mathbb{Z}_{n}}=\mathbb{Z}_{n} \backslash \mathbb{S}^{3}=: \mathbb{L}(n, 1)$.

Following [32], we come now to a central concept of this paper.

Definition 3.5 (topological T-duality). Let $\mathbb{T}_{\Lambda_{\mathrm{G}}}$ be a twisted torus which admits a non-trivial right action of the abelian Lie group $\mathbb{R}^{n}$ for some $n \geq 1$. The crossed product

$$
C\left(\mathbb{T}_{\Lambda_{\mathrm{G}}}\right) \rtimes_{\mathrm{rt}} \mathbb{R}^{n}
$$

is a $C^{*}$-algebraic $T$-dual of the twisted torus.

If the spectrum of the crossed product algebra $C\left(\mathbb{T}_{\Lambda_{\mathrm{G}}}\right) \rtimes_{\mathrm{rt}} \mathbb{R}^{n}$ is a Hausdorff topological space $X$ (for instance if it is Morita equivalent to a commutative $C^{*}$-algebra $C(X)$ ), then we say that $X$ is T-dual to the twisted torus $\mathbb{T}_{\Lambda_{\mathrm{G}}}$ and call $X$ a 'classical T-dual'; otherwise we say that the $\mathrm{T}$-dual of $\mathbb{T}_{\Lambda_{\mathrm{G}}}$ is a noncommutative space.

For Definition 3.5 to be a 'good' notion of T-duality, we should first explain

(a) in what precise sense $\mathbb{T}_{\Lambda_{\mathrm{G}}}$ and $C\left(\mathbb{T}_{\Lambda_{\mathrm{G}}}\right) \rtimes_{\mathrm{rt}} \mathbb{R}^{n}$ are 'equivalent', and

(b) how T-duality applied twice returns the original twisted torus.

The answers to both of these points turns out to be provided by working in a suitable category tailored to our treatment of topological T-duality.

\section{$3.2 \quad$ T-duality in the category $\mathscr{K} \mathscr{K}$}

The terminology 'topological T-duality' refers to a coarse equivalence at the level of topology; for $C^{*}$-algebras the topology is measured by K-theory. A more powerful refinement is provided by Kasparov's bivariant K-theory which constructs groups $\mathrm{KK}(\mathcal{A}, \mathcal{B})$ for any pair of separable $C^{*}$-algebras $\mathcal{A}$ and $\mathcal{B}$; when $\mathcal{A}=\mathbb{C}$, the group $\mathrm{KK}(\mathbb{C}, \mathcal{B}) \simeq \mathrm{K}(\mathcal{B})$ is the $\mathrm{K}$-theory group of $\mathcal{B}$. The cycles in Kasparov's groups $\operatorname{KK}(\mathcal{A}, \mathcal{B})$, called Kasparaov $\mathcal{A}-\mathcal{B}$ bimodules, are triples $(\mathcal{H}, \phi, T)$ where $\mathcal{H}$ is a right Hilbert $\mathcal{B}$-module, $\phi$ is a $*$-representation of $\mathcal{A}$ on $\mathcal{H}$, and $T \in \operatorname{End}_{\mathcal{B}}(\mathcal{H})$ is a $\mathcal{B}$ linear operator on $\mathcal{H}$, subject to certain compactness conditions; we do not provide further details of the definition here and instead refer to [6] for a concise review of KK-theory in the context that we shall use it in this paper. Kasparov bimodules may be thought of as generalizations of morphisms between $C^{*}$-algebras, in the sense that any algebra homomorphism $\phi: \mathcal{A} \rightarrow \mathcal{B}$ determines a class $[\phi] \in \mathrm{KK}(\mathcal{A}, \mathcal{B})$, represented by the $\mathcal{A}$ - $\mathcal{B}$-bimodule $(\mathcal{B}, \phi, 0)$.

A key feature of Kasparov's KK-theory is the composition product

$$
\otimes_{\mathcal{B}}: \operatorname{KK}(\mathcal{A}, \mathcal{B}) \times \mathrm{KK}(\mathcal{B}, \mathcal{C}) \longrightarrow \mathrm{KK}(\mathcal{A}, \mathcal{C})
$$

which is bilinear and associative. This product is compatible with the composition of morphisms $\phi: \mathcal{A} \rightarrow \mathcal{B}$ and $\psi: \mathcal{B} \rightarrow \mathcal{C}$ of $C^{*}$-algebras: $[\phi] \otimes_{\mathcal{B}}[\psi]=[\psi \circ \phi]$. It also makes $\operatorname{KK}(\mathcal{A}, \mathcal{A})$ into a ring with unit $1_{\mathcal{A}}=\left[\mathrm{id}_{\mathcal{A}}\right]$. We say that an element $\alpha \in \operatorname{KK}(\mathcal{A}, \mathcal{B})$ is invertible if there exists an element $\beta \in \operatorname{KK}(\mathcal{B}, \mathcal{A})$ such that $\alpha \otimes_{\mathcal{B}} \beta=1_{\mathcal{A}}$ and $\beta \otimes_{\mathcal{A}} \alpha=1_{\mathcal{B}}$.

An important special instance of Kasparov bimodules comes from Morita equivalence: Any Morita equivalence $\mathcal{A}-\mathcal{B}$ bimodule $\mathcal{M}$ is also a Kasparov bimodule $(\mathcal{M}, \phi, 0)$, with $\phi: \mathcal{A} \rightarrow$ $\operatorname{End}(\mathcal{M})$ the left action of $\mathcal{A}$, which defines an invertible class $[\mathcal{M}] \in \operatorname{KK}(\mathcal{A}, \mathcal{B})$ with inverse $[\overline{\mathcal{M}}] \in \operatorname{KK}(\mathcal{B}, \mathcal{A})$ given by the conjugate $\mathcal{B}-\mathcal{A}$ bimodule $\overline{\mathcal{M}}$. Generally, if there exists an invertible element $\alpha \in \operatorname{KK}(\mathcal{A}, \mathcal{B})$, then the algebras $\mathcal{A}$ and $\mathcal{B}$ are said to be $K K$-equivalent, and 
we write $\mathcal{A} \sim_{\mathrm{KK}} \mathcal{B}$. Thus Morita equivalence implies KK-equivalence, but the converse is not generally true. KK-equivalent algebras have isomorphic K-theory groups, but not necessarily homeomorphic spectra.

This refinement naturally suggests an approach to T-duality where the category of separable $C^{*}$-algebras with $*$-homomorphisms is replaced with an additive category $\mathscr{K} \mathscr{K}$, whose objects are again separable $C^{*}$-algebras but whose morphisms between any two objects $\mathcal{A}$ and $\mathcal{B}$ are given by the classes in $\operatorname{KK}(\mathcal{A}, \mathcal{B})$ (see, e.g., [7]). The composition product defines the composition law, and isomorphic algebras in this category are precisely the KK-equivalent algebras; in particular, Morita equivalent algebras are isomorphic as objects in $\mathscr{K} \mathscr{K}$. Our formulation and computations of topological T-duality will always take place in this category, and in this setting we can easily provide answers to points (a) and (b) below Definition 3.5 through

Theorem 3.6. If $\mathbb{T}_{\Lambda_{\mathrm{G}}}$ is a twisted torus with a non-trivial right action of $\mathbb{R}^{n}$, then there are isomorphisms in the category $\mathscr{K} \mathscr{K}$ given by the equivalences

(a) $C\left(\mathbb{T}_{\Lambda_{\mathrm{G}}}\right) \sim_{K K} C\left(\mathbb{T}_{\Lambda_{\mathrm{G}}}\right) \rtimes_{\mathrm{rt}} \mathbb{R}^{n}$ (up to a shift of degree $\left.n \bmod 2\right)$, and

(b) $\left(C\left(\mathbb{T}_{\Lambda_{\mathrm{G}}}\right) \rtimes_{\mathrm{rt}} \mathbb{R}^{n}\right) \rtimes_{\widehat{\mathrm{rt}}} \mathbb{R}^{n} \sim_{M} C\left(\mathbb{T}_{\Lambda_{\mathrm{G}}}\right)$.

Proof. The KK-equivalence (a) follows from the Connes-Thom isomorphism, formulated in the language of KK-theory [17]. The Morita equivalence (b) follows from Takai duality (Theorem 2.9).

Another virtue of the categorical setting is that it enables a general algebraic reformulation of the correspondence space construction, which for topological spaces 'geometrizes' the action of topological T-duality. In [7, Proposition 5.3] it is proven that, if $\mathcal{A}$ and $\mathcal{B}$ are separable $C^{*}$ algebras, then any class in $\operatorname{KK}(\mathcal{A}, \mathcal{B})$ can be represented by a 'noncommutative correspondence'. For this, we first recall, following [6,7], that KK-theory provides a definition of Gysin or "wrong way" homomorphisms on K-theory for $C^{*}$-algebras. If $\phi: \mathcal{A} \rightarrow \mathcal{B}$ is a morphism of separable $C^{*}$ algebras, a $K$-orientation is a functorial assignment of a corresponding element $\phi ! \in \operatorname{KK}(\mathcal{B}, \mathcal{A})$. If a K-orientation exists, we say that $\phi$ is K-oriented and call $\phi$ ! the associated Gysin element. The Gysin homomorphism on K-theory is now defined by $\phi_{!}:=(-) \otimes_{\mathcal{B}} \phi !: \mathrm{K}(\mathcal{B}) \rightarrow \mathrm{K}(\mathcal{A})$. We then slightly adapt the definition from [7] to the present context of Theorem 3.6.

Definition 3.7 (noncommutative correspondences). Let $\mathbb{T}_{\Lambda_{\mathrm{G}}}$ be a twisted torus which admits a non-trivial right action of $\mathbb{R}^{n}$, and let

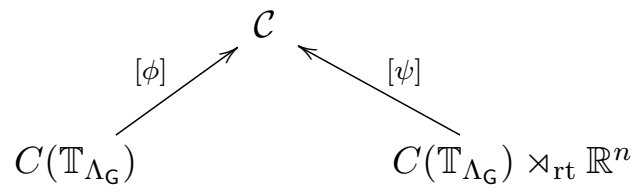

be a diagram in $\mathscr{K} \mathscr{K}$ whose arrows are induced by homomorphisms $\phi: C\left(\mathbb{T}_{\Lambda_{\mathrm{G}}}\right) \rightarrow \mathcal{C}$ and $\psi: C\left(\mathbb{T}_{\Lambda_{\mathrm{G}}}\right) \rtimes_{\mathrm{rt}} \mathbb{R}^{n} \rightarrow \mathcal{C}$ of separable $C^{*}$-algebras. Assume that $\psi$ is $\mathrm{K}$-oriented, and let $\psi ! \in$ $\operatorname{KK}\left(\mathcal{C}, C\left(\mathbb{T}_{\Lambda_{\mathrm{G}}}\right) \rtimes_{\mathrm{rt}} \mathbb{R}^{n}\right)$ be its corresponding Gysin element. The separable $C^{*}$-algebra $\mathcal{C}$ is a noncommutative correspondence if the associated element

$$
[\phi] \otimes_{\mathcal{C}} \psi ! \in \mathrm{KK}\left(C\left(\mathbb{T}_{\Lambda_{\mathrm{G}}}\right), C\left(\mathbb{T}_{\Lambda_{\mathrm{G}}}\right) \rtimes_{\mathrm{rt}} \mathbb{R}^{n}\right)
$$

is a KK-equivalence between the twisted torus and its $C^{*}$-algebraic T-dual.

Analogously to [4], we obtain a noncommutative correspondence by restricting the $\mathbb{R}^{n}$-action to the lattice $\mathbb{Z}^{n} \subset \mathbb{R}^{n}$. 
Proposition 3.8. The crossed product

$$
\mathcal{C}=C\left(\mathbb{T}_{\Lambda_{\mathrm{G}}}\right) \rtimes_{\left.\mathrm{rt}\right|_{\mathbb{Z}^{n}}} \mathbb{Z}^{n}
$$

is a noncommutative correspondence in the sense of Definition 3.\%.

Proof. We need to construct a diagram (3.1) in $\mathscr{K} \mathscr{K}$ for the crossed product. For this, note that for any dynamical system of the form $(\mathcal{A}, \Lambda, \alpha)$ where $\Lambda$ is a discrete group, there is a natural injection $j$ of the algebra $\mathcal{A}$ into the crossed product $\mathcal{A} \rtimes_{\alpha} \Lambda$ : given $a \in \mathcal{A}$, define the sequence $j(a) \in C_{\mathrm{c}}(\Lambda, \mathcal{A})$ by $j(a)_{\gamma}=a \delta_{\gamma, e}$ for $\gamma \in \Lambda$. It is easy to check, using the explicit formula for the convolution product, that the map $a \mapsto j(a)$ is an algebra monomorphism: $j(a) \star j(b)=j(a b)$ for $a, b \in \mathcal{A}$. In particular, there is a $C^{*}$-algebra injection

$$
j:\left.C\left(\mathbb{T}_{\Lambda_{\mathrm{G}}}\right) \longrightarrow C\left(\mathbb{T}_{\Lambda_{\mathrm{G}}}\right) \rtimes_{\mathrm{rt}}\right|_{\mathbb{Z}^{n}} \mathbb{Z}^{n} .
$$

Next we apply [20, Corollary 2.8] with $\mathbb{R}^{n}$ acting on $\mathbb{T}^{n}=\mathbb{R}^{n} / \mathbb{Z}^{n}$ by (right) translation and the diagonal action of $\mathbb{R}^{n}$ on $\mathbb{T}^{n} \times \mathbb{T}_{\Lambda_{\mathrm{G}}}$ to obtain an isomorphism

$$
C\left(\mathbb{T}^{n} \times \mathbb{T}_{\Lambda_{\mathrm{G}}}\right) \rtimes_{\mathrm{rt}} \mathbb{R}^{n} \simeq\left(C\left(\mathbb{T}_{\Lambda_{\mathrm{G}}}\right) \rtimes_{\left.\mathrm{rt}\right|_{\mathbb{Z}^{n}}} \mathbb{Z}^{n}\right) \otimes \mathcal{K}\left(\mathrm{L}^{2}\left(\mathbb{T}^{n}\right)\right)
$$

The projection $\mathbb{T}^{n} \times \mathbb{T}_{\Lambda_{\mathrm{G}}} \rightarrow \mathbb{T}_{\Lambda_{\mathrm{G}}}$ induces an injection $C_{\mathrm{c}}\left(\mathbb{R}^{n} \times \mathbb{T}_{\Lambda_{\mathrm{G}}}\right) \hookrightarrow C_{\mathrm{c}}\left(\mathbb{R}^{n} \times \mathbb{T}^{n} \times \mathbb{T}_{\Lambda_{\mathrm{G}}}\right)$ which preserves the convolution product, and we obtain a $C^{*}$-algebra monomorphism

$$
\psi^{\prime}: C\left(\mathbb{T}_{\Lambda_{\mathrm{G}}}\right) \rtimes_{\mathrm{rt}} \mathbb{R}^{n} \longrightarrow\left(\left.C\left(\mathbb{T}_{\Lambda_{\mathrm{G}}}\right) \rtimes_{\mathrm{rt}}\right|_{\mathbb{Z}^{n}} \mathbb{Z}^{n}\right) \otimes \mathcal{K}\left(\mathrm{L}^{2}\left(\mathbb{T}^{n}\right)\right)
$$

which is easily checked to be K-oriented since it is induced by a projection.

This gives algebra morphisms $\phi^{\prime}:=\iota \circ j: C\left(\mathbb{T}_{\Lambda_{\mathrm{G}}}\right) \rightarrow \mathcal{C} \otimes \mathcal{K}$ and $\psi^{\prime}: C\left(\mathbb{T}_{\Lambda_{\mathrm{G}}}\right) \rtimes_{\mathrm{rt}} \mathbb{R}^{n} \rightarrow \mathcal{C} \otimes \mathcal{K}$, where $\mathcal{C}=C\left(\mathbb{T}_{\Lambda_{\mathrm{G}}}\right) \rtimes_{\left.\mathrm{rt}\right|_{\mathbb{Z}^{n}}} \mathbb{Z}^{n}, \mathcal{K}$ denotes the $C^{*}$-algebra of compact operators on a separable Hilbert space, and $\iota: \mathcal{C} \rightarrow \mathcal{C} \otimes \mathcal{K}$ is the usual stabilization map. Taking the composition products of $\left[\phi^{\prime}\right]$ and $\left[\psi^{\prime}\right]$ with the Morita equivalence $\mathcal{C} \otimes \mathcal{K} \sim_{\mathrm{M}} \mathcal{C}$ then yields the required maps in (3.1).

When the spectrum of the $C^{*}$-algebraic T-dual is a Hausdorff space, we identify the correspondence space with the spectrum of $\mathcal{C}=\left.C\left(\mathbb{T}_{\Lambda_{\mathrm{G}}}\right) \rtimes_{\mathrm{rt}}\right|_{\mathbb{Z}^{n}} \mathbb{Z}^{n}$; otherwise $\mathcal{C}$ is a noncommutative space.

\subsection{Computational tools}

Let us now explain how to compute these $C^{*}$-algebraic T-duals in some special instances that will appear throughout the remainder of this paper. For certain actions of $\mathbb{R}$, we may compute the $C^{*}$-algebraic T-dual via

Proposition 3.9. Let $\mathbb{T}_{\Lambda_{G}}$ be a twisted torus equipped with an action of $\mathbb{R}$ for which every point has isotropy subgroup $\mathbb{Z}$. Let $T=\mathbb{T}_{\Lambda_{\mathrm{G}}} / \mathbb{R}$, and denote the corresponding principal circle bundle by p: $\mathbb{T}_{\Lambda_{\mathrm{G}}} \rightarrow T$. Then the $C^{*}$-algebraic T-dual $C\left(\mathbb{T}_{\Lambda_{\mathrm{G}}}\right) \rtimes_{\mathrm{rt}} \mathbb{R} \simeq \mathrm{CT}(\mathbb{T} \times T, \delta)$ is a continuoustrace algebra with spectrum $\mathbb{T} \times T$ and Dixmier-Douady class $\delta=\zeta \smile c_{1}(p) \in \mathrm{H}^{3}(\mathbb{T} \times T, \mathbb{Z})$, where $c_{1}(p) \in \mathrm{H}^{2}(T, \mathbb{Z})$ is the Chern class of the circle bundle and $\zeta$ is the standard generator of $\mathrm{H}^{1}(\mathbb{T}, \mathbb{Z}) \simeq \mathbb{Z}$.

Proof. This is just a straightforward adaptation of the statement of [39, Proposition 4.5].

In these instances, the T-dual of $\mathbb{T}_{\Lambda_{\mathrm{G}}}$ is the Hausdorff space $X=\mathbb{T} \times\left(\mathbb{T}_{\Lambda_{\mathrm{G}}} / \mathbb{R}\right)$ with a threeform ' $H$-flux' whose cohomology class is represented by $[H]=\zeta \smile c_{1}(p)$. 
More generally, suppose that the $\mathbb{R}^{n}$-action on $\mathbb{T}_{\Lambda_{G}}$ is induced by a free and proper right action of $\mathbb{R}^{n}$ on the covering group $G$ which commutes with the left action of the lattice $\Lambda_{\mathrm{G}}$ on G. We can then apply Green's theorem (Theorem 2.13) to get the Morita equivalence

$$
C\left(\mathbb{T}_{\Lambda_{\mathrm{G}}}\right) \rtimes_{\mathrm{rt}} \mathbb{R}^{n} \sim_{\mathrm{M}} C_{0}\left(\mathrm{G} / \mathbb{R}^{n}\right) \rtimes_{\mathrm{lt}} \Lambda_{\mathrm{G}}
$$

In this special case, we obtain an easy proof of Proposition 3.8: The inclusion $\mathbb{Z}^{n} \hookrightarrow \mathbb{R}^{n}$ of groups induces a monomorphism

$$
C_{0}\left(\mathrm{G} / \mathbb{R}^{n}\right) \rtimes_{\mathrm{lt}} \Lambda_{\mathrm{G}} \longrightarrow C_{0}\left(\mathrm{G} / \mathbb{Z}^{n}\right) \rtimes_{\mathrm{lt}} \Lambda_{\mathrm{G}} \sim_{\mathrm{M}} C\left(\mathbb{T}_{\Lambda_{\mathrm{G}}}\right) \rtimes_{\left.\mathrm{rt}\right|_{\mathbb{Z}^{n}}} \mathbb{Z}^{n}
$$

where in the last step we replaced $\mathbb{R}^{n}$ by its subgroup $\mathbb{Z}^{n}$ in (3.3). This gives monomorphisms (3.2) and $\left.C\left(\mathbb{T}_{\Lambda_{\mathrm{G}}}\right) \rtimes_{\mathrm{rt}} \mathbb{R}^{n} \rightarrow C\left(\mathbb{T}_{\Lambda_{\mathrm{G}}}\right) \rtimes_{\mathrm{rt}}\right|_{\mathbb{Z}^{n}} \mathbb{Z}^{n}$ in the category $\mathscr{K} \mathscr{K}$.

\subsection{Topological T-duality for the torus}

Let us now describe how our considerations reproduce the standard T-duality for tori. The simplest example of the T-duality scheme (3.3) is the case where $G=\mathbb{R}, \Lambda_{\mathrm{G}}=\mathbb{Z} \subset \mathbb{R}$, and $\mathrm{G} / \mathbb{R}=\{0\}$ with the obviously trivial $\Lambda_{\mathrm{G}}$-action. Then $\mathbb{T}_{\mathbb{Z}}=\mathbb{Z} \backslash \mathbb{R}=\mathbb{T}$ is a circle, and (3.3) with $n=1$ reads

$$
C(\mathbb{T}) \rtimes_{\mathrm{rt}} \mathbb{R} \sim_{\mathrm{M}} \mathbb{C} \rtimes \mathbb{Z}=C^{*}(\mathbb{Z}) \simeq C(\widetilde{\mathbb{T}}),
$$

where in the last passage we used the Fourier transform isomorphism $\mathcal{F}: C^{*}(\mathbb{Z}) \rightarrow C(\widetilde{\mathbb{T}})$; explicitly, if $a=\left\{a_{n}\right\}_{n \in \mathbb{Z}} \in C^{*}(\mathbb{Z})$, then $\mathcal{F}(a)(\chi)=\sum_{n \in \mathbb{Z}} a_{n} \mathrm{e}^{2 \pi \mathrm{in} \chi}$ so that $\mathcal{F}(a)$ is a function on the dual circle $\widetilde{\mathbb{T}}=\mathbb{R}^{*} / \mathbb{Z}^{*}$.

The generalization to T-duality along a single direction $i$ of a $d$-dimensional torus is straightforward. Let $\Lambda \simeq \mathbb{Z}^{d}$ be the lattice in $\mathbb{R}^{d}$ given by $\Lambda=\left\{\sum_{i=1}^{d} a_{i} \vec{e}_{i} \mid a_{1}, \ldots, a_{d} \in \mathbb{Z}\right\}$, where $\vec{e}_{1}, \ldots, \vec{e}_{d}$ is the standard basis of $\mathbb{R}^{d}$; this is the direct sum $\Lambda=\bigoplus_{i=1}^{d} \mathbb{Z} \vec{e}_{i}$. Let $\mathbb{R}_{i}$ be the subgroup of $\mathbb{R}^{d}$ linearly spanned by $\vec{e}_{i}$ and let $\mathbb{Z}_{i} \subset \mathbb{R}_{i}$ be the corresponding lattice; we write $\mathbb{T}_{i}=\mathbb{R}_{i} / \mathbb{Z}_{i}$ and decompose the $d$-torus $\mathbb{T}^{d}=\mathbb{R}^{d} / \Lambda$ as $\mathbb{T}^{d}=\mathbb{T}_{\hat{\imath}}^{d-1} \times \mathbb{T}_{i}$, where $\mathbb{T}_{\hat{\imath}}^{d-1}$ is the $(d-1)$-dimensional torus defined by omitting the $i$-th factors of $\mathbb{R}^{d}$ and $\Lambda$. Then $\mathbb{R}_{i}$ acts trivially on $\mathbb{T}_{\hat{\imath}}^{d-1}$ and we have

$$
\begin{aligned}
C\left(\mathbb{T}^{d}\right) \rtimes_{\mathrm{rt}} \mathbb{R}_{i} & =C\left(\mathbb{T}_{\hat{\imath}}^{d-1} \times \mathbb{T}_{i}\right) \rtimes_{\mathrm{rt}} \mathbb{R}_{i} \simeq\left(C\left(\mathbb{T}_{\hat{\imath}}^{d-1}\right) \otimes C\left(\mathbb{T}_{i}\right)\right) \rtimes_{\mathrm{id} \otimes \mathrm{rt}} \mathbb{R}_{i} \\
& \simeq C\left(\mathbb{T}_{\hat{\imath}}^{d-1}\right) \otimes\left(C\left(\mathbb{T}_{i}\right) \rtimes_{\mathrm{rt}} \mathbb{R}_{i}\right) \sim_{\mathrm{M}} C\left(\mathbb{T}_{\hat{\imath}}^{d-1}\right) \otimes C\left(\widetilde{\mathbb{T}}_{i}\right) \\
& \simeq C\left(\mathbb{T}_{\hat{\imath}}^{d-1} \times \widetilde{\mathbb{T}}_{i}\right)=C\left(\mathbb{T}_{\hat{\imath}}^{d}\right),
\end{aligned}
$$

where $\mathbb{T}_{\tilde{\imath}}^{d}:=\mathbb{T}_{\hat{\imath}}^{d-1} \times \widetilde{\mathbb{T}}_{i}$. This is the expected action of the $i$-th factorized T-duality, and in this way we have thus reproduced the standard rules for T-duality of tori. In fact, in this case we can use Proposition 3.9 to strengthen the statement of topological T-duality: Every point of $\mathbb{T}^{d}$ has isotropy group $\mathbb{Z}$ under the action of $\mathbb{R}_{i}$, and the corresponding circle bundle $p: \mathbb{T}^{d} \rightarrow \mathbb{T}_{\hat{\imath}}^{d-1}$ is trivial, so the $C^{*}$-algebraic $\mathrm{T}$-dual of $\mathbb{T}^{d}$ is a continuous-trace algebra with spectrum $\mathbb{T}_{\tilde{\imath}}^{d}$ and trivial Dixmier-Douady class. Hence the Morita equivalence in (3.4) can be replaced by a stable isomorphism.

By iterating these T-duality transformations one can perform T-dualities along multiple directions of a $d$-dimensional torus. In particular, iterating the procedure $d$ times and using Theorem 2.6 we end up with the full T-duality

$$
C\left(\mathbb{T}^{d}\right) \rtimes_{\mathrm{rt}} \mathbb{R}^{d} \sim_{\mathrm{M}} C\left(\widetilde{\mathbb{T}}^{d}\right)
$$


where $\widetilde{\mathbb{T}}^{d}=\left(\mathbb{R}^{d}\right)^{*} / \Lambda^{*}$ is the dual torus with $\Lambda^{*}$ the dual lattice in the dual vector space $\left(\mathbb{R}^{d}\right)^{*}$. This can also be obtained directly by setting $G=\mathbb{R}^{d}, \Lambda_{\mathrm{G}}=\Lambda \subset \mathbb{R}^{d}$ and $\mathrm{G} / \mathbb{R}^{d}=\{0\}$ in (3.3) with $n=d$, and by using the Fourier transforms in all directions $\vec{e}_{1}, \ldots, \vec{e}_{d}$.

Finally, let us consider the correspondence space construction. For the $i$-th factorized Tduality, this is obtained by restricting the action of $\mathbb{R}_{i}$ to the lattice $\mathbb{Z}_{i} \subset \mathbb{R}_{i}$. The action of the group $\mathbb{Z}_{i}$ on the algebra of functions $C\left(\mathbb{T}^{d}\right)$ is trivial and so we get isomorphisms

$$
C\left(\mathbb{T}^{d}\right) \rtimes_{\mathrm{rt}} \mathbb{Z}_{i} \simeq C\left(\mathbb{T}^{d}\right) \otimes C^{*}\left(\mathbb{Z}_{i}\right) \simeq C\left(\mathbb{T}^{d}\right) \otimes C\left(\widetilde{\mathbb{T}}_{i}\right) \simeq C\left(\mathbb{T}^{d} \times \widetilde{\mathbb{T}}_{i}\right)
$$

This results in the noncommutative correspondence induced by the diagram

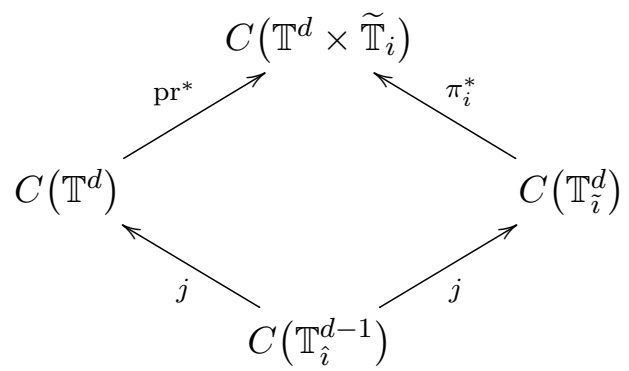

where pr: $\mathbb{T}^{d} \times \widetilde{\mathbb{T}}_{i} \rightarrow \mathbb{T}^{d}$ is the projection to the first factor and $\pi_{i}: \mathbb{T}^{d} \times \widetilde{\mathbb{T}}_{i} \rightarrow \mathbb{T}_{\tilde{\imath}}^{d}$ omits the $i$-th factor of $\mathbb{T}^{d}$. The algebra inclusions $j$ of $C\left(\mathbb{T}_{\hat{\imath}}^{d-1}\right)$ are induced by the trivial circle bundle projections $\mathbb{T}^{d} \rightarrow \mathbb{T}_{\hat{\imath}}^{d-1}$ and $\mathbb{T}_{\tilde{\imath}}^{d} \rightarrow \mathbb{T}_{\hat{\imath}}^{d-1}$.

By either iterating this construction using Theorem 2.6 or by direct calculation, the correspondence space for a full T-duality is obtained by restricting the action of $\mathbb{R}^{d}$ from (3.5) to the lattice $\Lambda \subset \mathbb{R}^{d}$, and we analogously find

$$
C\left(\mathbb{T}^{d}\right) \rtimes_{\mathrm{rt}} \Lambda \simeq C\left(\mathbb{T}^{d} \times \widetilde{\mathbb{T}}^{d}\right)
$$

Thus the crossed product with the lattice of periods $\Lambda$ defining the $d$-torus $\mathbb{T}^{d}$ recovers the doubled torus $\mathbb{T}^{d} \times \widetilde{\mathbb{T}}^{d}$ which is the correspondence space for the smooth Fourier-Mukai transform, wherein a full or factorized T-duality has a geometric interpretation as an element of its automorphism group $\mathrm{GL}(2 d, \mathbb{Z})$.

\subsection{Topological T-duality for orbifolds}

Let $\mathrm{G}$ be a compact connected semisimple Lie group of $\operatorname{rank} r$, and let $\Gamma \subset \mathrm{G}$ be a finite subgroup. The maximal torus $\mathrm{T}=\mathrm{U}(1)^{r} \simeq \mathbb{R}^{r} / \mathbb{Z}^{r}$ of $\mathrm{G}$ carries a natural action of $\mathbb{R}_{i}$ by translation along the $i$-th direction for $i=1, \ldots, r$, and we can apply a fibrewise T-duality to the principal torus bundle $G \rightarrow G / T$. Under this $\mathbb{R}$-action every point of $G$ has isotropy subgroup $\mathbb{Z}$, and the action descends to the smooth orbifold $\mathbb{T}_{\Gamma}=\Gamma \backslash \mathrm{G}$. Then the quotient map $p_{i}: \mathbb{T}_{\Gamma} \rightarrow \mathbb{T}_{\Gamma} / \mathbb{R}_{i}$ is the circle fibration $\mathbb{T}_{\Gamma} \rightarrow \mathbb{T}_{\Gamma} / \mathrm{U}(1)_{i}$, where $\mathrm{T}=\mathrm{U}(1)_{\hat{\imath}}^{r-1} \times \mathrm{U}(1)_{i}$, and by Proposition 3.9 the $C^{*}$-algebraic T-dual

$$
C(\Gamma \backslash \mathrm{G}) \rtimes_{\mathrm{rt}} \mathbb{R}_{i} \simeq \mathrm{CT}\left(\Gamma \backslash \mathrm{G} / \mathrm{U}(1)_{i} \times \widetilde{\mathbb{T}}_{i}, \delta_{i}\right)
$$

of the orbifold $\mathbb{T}_{\Gamma}$ is a continuous-trace algebra with spectrum $\mathbb{T}_{\Gamma} / \mathrm{U}(1)_{i} \times \widetilde{\mathbb{T}}_{i}$ and DixmierDouady class $\delta_{i}=c_{1}\left(p_{i}\right) \smile \zeta_{i}$.

In the rank one case, this T-duality is well-known (see, e.g., [3]): Then $\mathrm{G}=\mathrm{SU}(2)$ which we regard as the three-sphere $\mathbb{S}^{3}$, and for $\Gamma=\mathbb{Z}_{n} \subset \mathrm{T}=\mathrm{U}(1)$ the twisted torus is the lens space $\mathbb{L}(n, 1)$. The quotient map $p: \mathbb{L}(n, 1) \rightarrow \mathbb{S}^{2}$ is a circle bundle whose Chern class $c_{1}(p)$ is equal to $n$ times the standard generator of $\mathrm{H}^{2}\left(\mathbb{S}^{2}, \mathbb{Z}\right) \simeq \mathbb{Z}$, and applying (3.6) we find that the 
$C^{*}$-algebraic T-dual of $\mathbb{L}(n, 1)$ is a continuous-trace algebra whose spectrum is the trivial circle bundle $\mathbb{L}(0,1)=\mathbb{S}^{2} \times \widetilde{\mathbb{T}}$ and whose Dixmier-Douady class $\delta$ is $n$ times the standard generator of $\mathrm{H}^{3}\left(\mathbb{S}^{2} \times \widetilde{\mathbb{T}}, \mathbb{Z}\right) \simeq \mathbb{Z}$.

Generally, the correspondence space construction is obtained by noting that, since the isotropy subgroup for any point of the $\mathbb{R}_{i}$-action is $\mathbb{Z}_{i} \subset \mathbb{R}_{i}$, the group $\mathbb{Z}_{i}$ acts trivially on the algebra $C\left(\mathbb{T}_{\Gamma}\right)$ and there are isomorphisms

$$
C\left(\mathbb{T}_{\Gamma}\right) \rtimes_{\mathrm{rt}} \mathbb{Z}_{i} \simeq C\left(\mathbb{T}_{\Gamma}\right) \otimes C^{*}\left(\mathbb{Z}_{i}\right) \simeq C\left(\mathbb{T}_{\Gamma}\right) \otimes C\left(\widetilde{\mathbb{T}}_{i}\right) \simeq C\left(\mathbb{T}_{\Gamma} \times \widetilde{\mathbb{T}}_{i}\right) .
$$

Let pr: $\mathbb{T}_{\Gamma} \times \widetilde{\mathbb{T}}_{i} \rightarrow \mathbb{T}_{\Gamma}$ be the projection to the first factor. Since $H^{2}(G, \mathbb{Z})=0$, Künneth's theorem implies

$$
\left(p_{i} \times \mathrm{id}_{\widetilde{\mathbb{T}}_{i}}\right)^{*}\left(c_{1}\left(p_{i}\right) \smile \zeta_{i}\right)=0 \in \mathrm{H}^{3}\left(\mathbb{T}_{\Gamma} \times \widetilde{\mathbb{T}}_{i}, \mathbb{Z}\right),
$$

and hence the algebra $C T\left(\mathbb{T}_{\Gamma} \times \widetilde{\mathbb{T}}_{i},\left(p_{i} \times \mathrm{id}_{\widetilde{\mathbb{T}}_{i}}\right)^{*} \delta_{i}\right)$ is isomorphic to $C\left(\mathbb{T}_{\Gamma} \times \widetilde{\mathbb{T}}_{i}\right) \otimes \mathcal{K}$. Then there is the noncommutative correspondence

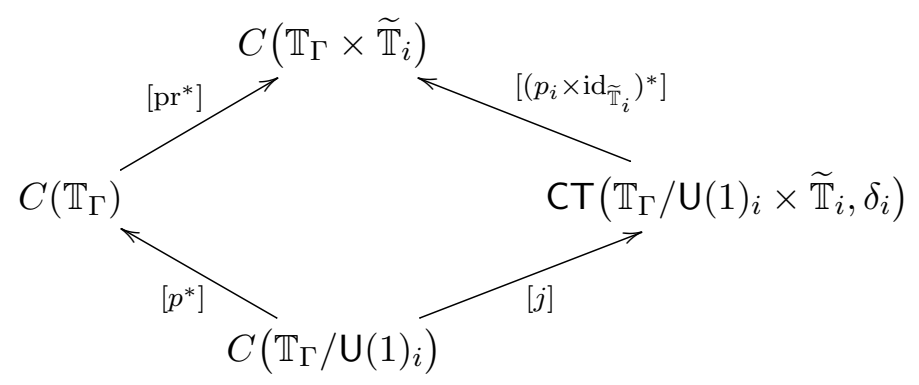

as a diagram in the category $\mathscr{K} \mathscr{K}$.

\section{Topological T-duality for almost abelian solvmanifolds}

A large class of twisted tori of interest as string compactifications come in the form of fibrations over tori. These are the solvmanifolds which are based on solvable groups $\mathrm{G}$ and generalize the nilmanifolds discussed in Example 3.3. The fibrations underlying these twisted tori are called Mostow bundles [35], and we are particularly interested in the cases where the Mostow bundle is a torus bundle. A good source for the material used in this section is [2] (see also [10, 50]).

\subsection{Mostow bundles}

Let $\mathrm{G}$ be a connected and simply-connected solvable Lie group. Recall that its nilradical $\mathrm{N}$ is the maximal connected nilpotent normal subgroup. It has $\operatorname{dimension} \operatorname{dim} N \geq \frac{1}{2} \operatorname{dim} \mathrm{G}$.

We first consider the case $\operatorname{dim} N=\operatorname{dim} G$. Then $N=G$ and the group $G$ is nilpotent. In this case, under the conditions discussed in Example 3.3, there exists a lattice $\Lambda_{\mathrm{G}} \subset \mathrm{G}$ and the twisted torus $\mathbb{T}_{\Lambda_{G}}$ is a nilmanifold. If $G$ is abelian then $\mathbb{T}_{\Lambda_{G}}$ is a torus. If $G$ is non-abelian then there is a group extension

$$
1 \longrightarrow[\mathrm{G}, \mathrm{G}] \longrightarrow \mathrm{G} \stackrel{\pi}{\longrightarrow} \mathrm{G}_{\mathrm{ab}} \longrightarrow 1
$$

of its commutator subgroup $[\mathrm{G}, \mathrm{G}]$, and both $\Lambda_{\mathrm{G}} \cap[\mathrm{G}, \mathrm{G}]$ and $\pi\left(\Lambda_{\mathrm{G}}\right)$ are lattices in the nilpotent Lie group $[G, G]$ and the abelianization $G_{a b}:=[G, G] \backslash G$ of $G$, respectively [11]. This exhibits the twisted torus $\mathbb{T}_{\Lambda_{\mathrm{G}}}=\Lambda_{\mathrm{G}} \backslash \mathrm{G}$ as a fibration over the torus $\pi\left(\Lambda_{\mathrm{G}}\right) \backslash \mathrm{G}_{\mathrm{ab}}$ with nilmanifold fibres,

$$
\left(\Lambda_{\mathrm{G}} \cap[\mathrm{G}, \mathrm{G}]\right) \backslash[\mathrm{G}, \mathrm{G}] \longrightarrow \mathbb{T}_{\Lambda_{\mathrm{G}}} \longrightarrow \pi\left(\Lambda_{\mathrm{G}}\right) \backslash \mathrm{G}_{\mathrm{ab}} .
$$

If $[G, G]$ is an abelian Lie group then the twisted torus is a torus bundle over a torus. 
Suppose now that the group $\mathrm{G}$ is not nilpotent. Then $N \backslash G$ is a non-trivial abelian Lie group. If $\mathrm{G}$ admits a lattice $\Lambda_{\mathrm{G}}$, then $\Lambda_{\mathrm{N}}:=\Lambda_{\mathrm{G}} \cap \mathrm{N}$ is a lattice in $\mathrm{N}$ and $\Lambda_{\mathrm{G}} \mathrm{N}=\mathrm{N} \Lambda_{\mathrm{G}}$ is a closed subgroup of $G$, so $\Lambda_{G} N \backslash G$ is a torus. The twisted torus $\mathbb{T}_{\Lambda_{G}}=\Lambda_{G} \backslash G$ is then a fibration over this torus with fibre the nilmanifold $\Lambda_{\mathrm{N}} \backslash \mathrm{N}=\Lambda_{\mathrm{G}} \backslash \Lambda_{\mathrm{G}} \mathrm{N}$. This bundle is called the Mostow bundle [35]. We summarise these statements as

Theorem 4.1 (Mostow bundles). Let $\Lambda_{\mathrm{G}}$ be a lattice in a connected and simply-connected solvable Lie group $\mathrm{G}$ and $\mathbb{T}_{\Lambda_{\mathrm{G}}}=\Lambda_{\mathrm{G}} \backslash \mathrm{G}$ the associated solvmanifold. Let $\mathrm{N}$ be the nilradical of $\mathrm{G}$. Then $\Lambda_{\mathrm{G}} \mathrm{N}$ is a closed subgroup of $\mathrm{G}, \Lambda_{\mathrm{N}}:=\Lambda_{\mathrm{G}} \cap \mathrm{N}$ is a lattice in $\mathrm{N}$, and $\Lambda_{\mathrm{G}} \mathrm{N} \backslash \mathrm{G}$ is a torus. It follows that the twisted torus $\mathbb{T}_{\Lambda_{\mathrm{G}}}$ is a fibration over this torus with nilmanifold fibre:

$$
\Lambda_{\mathrm{N}} \backslash \mathrm{N}=\Lambda_{\mathrm{G}} \backslash \Lambda_{\mathrm{G}} \mathrm{N} \longrightarrow \mathbb{T}_{\Lambda_{\mathrm{G}}} \longrightarrow \Lambda_{\mathrm{G}} \mathrm{N} \backslash \mathrm{G} .
$$

Remark 4.2. The structure group of the Mostow bundle is $\Lambda_{\mathrm{G}_{0}} \backslash \Lambda_{\mathrm{G}} \mathrm{N}$, where $\Lambda_{\mathrm{G}_{0}}$ is the largest subgroup of $\Lambda_{\mathrm{G}}$ which is normal in $\Lambda_{\mathrm{G}} \mathrm{N}$ (cf. [2]). In particular, if $\Lambda_{\mathrm{G}}=\Lambda_{\mathrm{G}_{0}}$ then the Mostow bundle is a principal $\Lambda_{\mathrm{G}} \backslash \Lambda_{\mathrm{G}} \mathrm{N}$-bundle. In this case there is a well-defined left $\Lambda_{\mathrm{G}} \mathrm{N}$-action on $\mathbb{T}_{\Lambda_{\mathrm{G}}}=\Lambda_{\mathrm{G}} \backslash \mathrm{G}$ and each point has isotropy subgroup $\Lambda_{\mathrm{G}}$, so that the induced $\Lambda_{\mathrm{G}} \backslash \Lambda_{\mathrm{G}} \mathrm{N}$-action is principal.

If the solvable Lie group $\mathrm{G}$ admits an abelian normal subgroup $\mathrm{V}$, then $\Lambda_{\mathrm{G}} \mathrm{V}=\mathrm{V} \Lambda_{\mathrm{G}}$ is a subgroup of $\mathrm{G}$; if $\Lambda_{\mathrm{G}}$ is normal in $\Lambda_{\mathrm{G}} \mathrm{V}$, then the Mostow bundle construction can be refined via an intermediate step involving a principal torus bundle over a second solvmanifold. Adapting [2, Theorem 3.6] we have

Proposition 4.3. Let $\mathrm{G}$ be a connected and simply-connected solvable Lie group and $\Lambda_{\mathrm{G}}$ a lattice in $\mathrm{G}$. Let $\mathrm{V}$ be a closed normal abelian Lie subgroup of $\mathrm{G}$ such that $\Lambda_{\mathrm{G}}$ is normal in $\mathrm{V} \Lambda_{\mathrm{G}}$. If $\mathrm{V}^{\mathbb{Z}}:=\Lambda_{\mathrm{G}} \cap \mathrm{V}$ is a lattice in $\mathrm{V}$, then $\Lambda_{\mathrm{G}} \backslash \Lambda_{\mathrm{G}} \mathrm{V}$ is a torus and the solvmanifold $\mathbb{T}_{\Lambda_{\mathrm{G}}}=\Lambda_{\mathrm{G}} \backslash \mathrm{G}$ is the total space of the principal torus bundle

$$
\Lambda_{\mathrm{G}} \backslash \Lambda_{\mathrm{G}} \mathrm{V} \longrightarrow \mathbb{T}_{\Lambda_{\mathrm{G}}} \longrightarrow \Lambda_{\mathrm{G}} \mathrm{V} \backslash \mathrm{G},
$$

with base the solvmanifold $\mathbb{T}_{\Lambda_{\mathrm{G}}}=\Lambda_{\mathrm{G}} \backslash \mathrm{G}^{\vee}:=\left(\mathrm{V}^{\mathbb{Z}} \backslash \Lambda_{\mathrm{G}}\right) \backslash(\mathrm{V} \backslash \mathrm{G})=\Lambda_{\mathrm{G}} \mathrm{V} \backslash \mathrm{G}$. There is moreover a double fibration

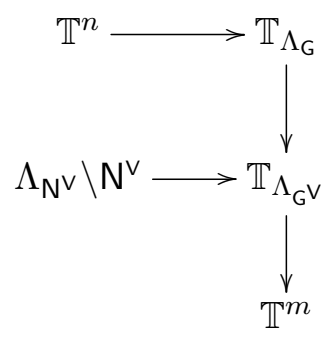

where $n=\operatorname{dim} \mathrm{V}, m=\operatorname{dim}\left(\mathrm{N}^{\vee} \backslash \mathrm{G}^{\vee}\right), \mathrm{N}^{\vee}$ is the nilradical of $\mathrm{G}^{\vee}$ and $\Lambda_{\mathrm{N}^{\vee}}=\mathrm{N}^{\vee} \cap \Lambda_{\mathrm{G}^{\vee}}$ the associated lattice.

Proof. Let $\mathrm{p}: \mathrm{G} \rightarrow \mathrm{V} \backslash \mathrm{G}$ be the canonical projection. Since $\mathrm{V}$ is normal in $\mathrm{G}$, and $\Lambda_{\mathrm{G}}$ and $\Lambda_{\mathrm{G}} \cap \mathrm{V}$ are lattices in $\mathrm{G}$ and $\mathrm{V}$, respectively, by [11, Lemma 5.1.4(a)] it follows that $\mathrm{p}\left(\Lambda_{\mathrm{G}}\right)$ is a lattice in $\mathrm{V} \backslash \mathrm{G}$. Hence $\mathrm{p}^{-1}\left(\mathrm{p}\left(\Lambda_{\mathrm{G}}\right)\right)=\mathrm{V} \Lambda_{\mathrm{G}}=\Lambda_{\mathrm{G}} \mathrm{V}$ is closed in $\mathrm{G}$, and $\pi: \mathrm{G} \rightarrow \Lambda_{\mathrm{G}} \mathrm{V} \backslash \mathrm{G}$ is a bundle. By [49, Section 7.4] (adapted to the smooth case), since $\Lambda_{\mathrm{G}}$ is a closed normal subgroup of $\Lambda_{\mathrm{G}} \mathrm{V}$, it follows that $\Lambda_{\mathrm{G}} \backslash \mathrm{G} \rightarrow \Lambda_{\mathrm{G}} \mathrm{V} \backslash \mathrm{G}$ is a principal $\Lambda_{\mathrm{G}} \backslash \Lambda_{\mathrm{G}} \mathrm{V}$-bundle (or in other words, Remark 4.2 holds as well under the present hypotheses). The fiber is a torus because $V$ is abelian, $\Lambda_{\mathrm{G}} \backslash \Lambda_{\mathrm{G}} \mathrm{V}=\mathrm{V} \mathbb{Z} \backslash \mathrm{V}=\mathbb{T}^{n}$, with $n=\operatorname{dim} \mathrm{V}$. Moreover, because $\mathrm{V}$ is normal in $\mathrm{G}$, there is a canonical action of the group $\mathrm{V}^{\mathbb{Z}} \backslash \Lambda_{\mathrm{G}}$ on the connected and simply-connected solvable Lie group $\mathrm{V} \backslash \mathrm{G}$ (given by $\left(\mathrm{V}^{\mathbb{Z}} \lambda\right)(\mathrm{V} g)=\mathrm{V}(\lambda g)$ for $\lambda \in \Lambda_{\mathrm{G}}$ and $\left.g \in \mathrm{G}\right)$, so that $\Lambda_{\mathrm{G}^{\vee}} \backslash \mathrm{G}^{\vee}:=\left(\mathrm{V} \mathbb{Z} \backslash \Lambda_{\mathrm{G}}\right) \backslash(\mathrm{V} \backslash \mathrm{G})$ is a solvmanifold. It is then easily proven that $\left(\mathrm{V} \backslash \Lambda_{\mathrm{G}}\right) \backslash(\mathrm{V} \backslash \mathrm{G})=\Lambda_{\mathrm{G}} \mathrm{V} \backslash \mathrm{G}$. The double fibration (4.1) follows immediately from Theorem 4.1 applied to $\mathbb{T}_{\Lambda_{\mathrm{G}}}=\Lambda_{\mathrm{G}^{\vee}} \backslash \mathrm{G}^{\vee}$. 
Remark 4.4. An equivalent form for the double fibration (4.1) is given by

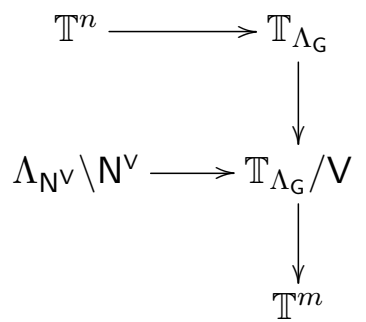

which is obtained by observing that $\Lambda_{\mathrm{G}} \mathrm{V} \backslash \mathrm{G}=\Lambda_{\mathrm{G}} \backslash \mathrm{G} / \mathrm{V}$ since $\mathrm{V}$ is normal in $\mathrm{G}$. Notice also that $\mathrm{G} / \mathrm{V}=\mathrm{V} \backslash \mathrm{G}$ and $\Lambda_{\mathrm{G}} / \mathrm{V}^{\mathbb{Z}}=\mathrm{V}^{\mathbb{Z}} \backslash \Lambda_{\mathrm{G}}$ (because $\mathrm{V}^{\mathbb{Z}}=\Lambda_{\mathrm{G}} \cap \mathrm{V}$ is normal in $\Lambda_{\mathrm{G}}$ ), and moreover $\Lambda_{\mathrm{G}} \backslash \mathrm{G}^{\vee}=\left(\mathrm{V}^{\mathbb{Z}} \backslash \Lambda_{\mathrm{G}}\right) \backslash(\mathrm{V} \backslash \mathrm{G})=\left(\Lambda_{\mathrm{G}} / \mathrm{V}^{\mathbb{Z}}\right) \backslash(\mathrm{G} / \mathrm{V})$.

\subsection{Almost abelian solvmanifolds}

In contrast to the case of nilpotent groups, there is no simple criterion for the existence of a lattice in a general connected and simply-connected solvable Lie group, as is required to define a corresponding twisted torus. To formulate such a criterion, we specialise to almost abelian solvable groups: these are the solvable Lie groups $\mathrm{G}$ of dimension $d$ whose nilradical $\mathrm{N}$ has codimension one and is abelian:

$$
\mathrm{N} \simeq \mathbb{R}^{d-1}
$$

Then G has the structure of a semi-direct product

$$
\mathrm{G}=\mathrm{N} \rtimes_{\varphi} \mathbb{R}
$$

for a continuous one-parameter left group action $\varphi: \mathbb{R} \rightarrow \operatorname{Aut}(\mathrm{N})$; concretely, $\varphi$ is given by the adjoint action of the one-dimensional subgroup $H=\mathbb{R}$ on $N$ in the group $G$ (cf. Section 2.2). This exhibits $\mathrm{G}$ as a nontrivial group extension

$$
1 \longrightarrow \mathrm{N} \longrightarrow \mathrm{G} \longrightarrow \mathbb{R} \longrightarrow 1 \text {. }
$$

We can regard the one-parameter group action $\varphi$ as a matrix $\varphi_{x} \in \mathrm{GL}(d-1, \mathbb{R})$ for each $x \in \mathbb{R}$ acting on the vector space $N$, which we identify with $\mathbb{R}^{d-1}$ via a choice of basis $\vec{e}_{1}, \ldots, \vec{e}_{d-1}$. Since $\varphi_{0}=\mathbb{1}_{d-1}$ and $\varphi_{x}$ is always non-singular, it follows from the continuity of $\varphi$ and the determinant that $\operatorname{det} \varphi_{x}>0$ for all $x \in \mathbb{R}$. Then $\mathrm{G}$ admits a lattice $\Lambda_{\mathrm{G}}$ if and only if there exists $x_{0} \in \mathbb{R}^{\times}$ such that $\varphi_{x_{0}}$ is conjugate to an integer matrix $\mathrm{M} \in \mathrm{SL}(d-1, \mathbb{Z})$ :

$$
\Sigma^{-1} \varphi_{x_{0}} \Sigma=\mathrm{M}
$$

for some $\Sigma \in \mathrm{GL}(d-1, \mathbb{R})$. In this case the twisted torus $\mathbb{T}_{\Lambda_{\mathrm{G}}}=\Lambda_{\mathrm{G}} \backslash \mathrm{G}$ is called an almost abelian solvmanifold. The condition (4.2) strongly restricts the homomorphisms $\varphi: \mathbb{R} \rightarrow \operatorname{Aut}(\mathrm{N})$; in particular, it requires that the characteristic polynomial of $\varphi_{x_{0}}$ has integer coefficients. In this case the lattice (in the standard basis $\vec{e}_{1}, \ldots, \vec{e}_{d-1}$ ) is given by

$$
\Lambda_{\mathrm{G}}=\Sigma \cdot \mathbb{Z}^{d-1} \rtimes_{\left.\varphi\right|_{x_{0}} \mathbb{Z}} x_{0} \mathbb{Z}
$$

which correspondingly sits as a nontrivial group extension

$$
1 \longrightarrow \Sigma \cdot \mathbb{Z}^{d-1} \longrightarrow \Lambda_{\mathrm{G}} \longrightarrow x_{0} \mathbb{Z} \longrightarrow 1 \text {. }
$$

Then $\Lambda_{\mathrm{N}} \backslash \mathrm{N} \simeq \mathbb{T}^{d-1}$, and the corresponding Mostow bundle realises the twisted torus $\mathbb{T}_{\Lambda_{\mathrm{G}}}$ as a torus bundle over a circle $\Lambda_{\mathrm{G}} \mathrm{N} \backslash \mathrm{G} \simeq \mathbb{T}$, whose monodromy is specified by the matrix $\mathrm{M}$ in the 
mapping class group $\mathrm{SL}(d-1, \mathbb{Z})$ of orientation-preserving automorphisms up to homotopy of the torus fibres $\mathbb{T}^{d-1}$.

For an almost abelian solvmanifold we can make the twisted torus construction more concrete by choosing the global coordinates $(z, x) \in \mathbb{R}^{d-1} \times \mathbb{R}$ on the group manifold (associated with the basis $\left.\vec{e}_{1}, \ldots, \vec{e}_{d-1}\right)$. The group multiplication of the semi-direct product $G=\mathbb{R}^{d-1} \rtimes_{\varphi} \mathbb{R}$ is

$$
(z, x)\left(z^{\prime}, x^{\prime}\right)=\left(z+\varphi_{x} \cdot z^{\prime}, x+x^{\prime}\right)
$$

where we used $\varphi_{x} \varphi_{x^{\prime}}=\varphi_{x+x^{\prime}}$, and the inverse of a group element is

$$
(z, x)^{-1}=\left(-\varphi_{-x} \cdot z,-x\right)
$$

where we used $\varphi_{x}^{-1}=\varphi_{-x}$. The twisted torus is defined as the quotient $\mathbb{T}_{\Lambda_{\mathrm{G}}}=\Lambda_{\mathrm{G}} \backslash \mathrm{G}$ which is generated by the equivalence relation $(z, x) \sim\left(\Sigma \cdot \gamma, x_{0} \alpha\right)(z, x)$ for all $(\gamma, \alpha) \in \mathbb{Z}^{d-1} \times \mathbb{Z}$. The global structure of $\mathbb{T}_{\Lambda_{\mathrm{G}}}$ is generated by the simultaneous local coordinate identifications under the action of the elements $\left(\Sigma \cdot \gamma, x_{0} \alpha\right)$ of $\Lambda_{\mathrm{G}}$ given by

$$
\begin{aligned}
& (z, x) \longmapsto(z+\Sigma \cdot \gamma, x), \\
& (z, x) \longmapsto\left(\Sigma \mathbb{M}^{\alpha} \Sigma^{-1} \cdot z, x+x_{0} \alpha\right) .
\end{aligned}
$$

These identifications explicitly exhibit the twisted torus as a torus bundle over a circle, with local fiber coordinates $z \in \mathbb{T}^{d-1}$ and base coordinate $x \in \mathbb{T}$, whose monodromy is specified by the matrix $\mathrm{M} \in \mathrm{SL}(d-1, \mathbb{Z})$, and whose periods are given respectively by $\Sigma \in \mathrm{GL}(d-1, \mathbb{R})$ and $x_{0} \in \mathbb{R}^{\times}$.

\section{$4.3 \quad C^{*}$-algebra bundles}

Mostow bundles and their $C^{*}$-algebraic T-duals can be grouped together under the general heading of ' $C^{*}$-algebra bundles', which encompasses the notions of $C_{0}(X)$-algebras and $C^{*}$ bundles, as we now explain; see Section 8.1 and Appendix $\mathrm{C}$ of [52] for further details. Let $X$ be a locally compact Hausdorff space. There are two equivalent notions for the $C^{*}$-algebra analogue of a fibre bundle over $X$.

A $C_{0}(X)$-algebra is a $C^{*}$-algebra $\mathcal{A}$ equipped with a nondegenerate injection $\iota$ of $C_{0}(X)$ into the centre of its multiplier algebra, called the structure map. For $\mathrm{f} \in C_{0}(X)$ and $a \in \mathcal{A}$, we abbreviate $\iota(\mathrm{f}) a$ by $\mathrm{f} \cdot a$. This endows $\mathcal{A}$ with a $C_{0}(X)$-bimodule structure.

Given a family $\mathcal{B}=\left(\mathcal{B}_{x}\right)_{x \in X}$ of $C^{*}$-algebras, a section of $\mathcal{B}$ is a map $s: X \rightarrow \mathcal{B}$ such that $s(x) \in \mathcal{B}_{x}$ for all $x \in X$; we denote the space of sections of $\mathcal{B}$ which vanish at infinity by $\Gamma_{0}(\mathcal{B})$. The family $\mathcal{B}$ is then called a $C^{*}$-bundle over $X$ with fibres $\mathcal{B}_{x}$ if the following conditions are satisfied:

- $\Gamma_{0}(\mathcal{B})$ is a $C^{*}$-algebra under pointwise operations and the supremum norm;

- $\mathcal{B}_{x}=\left\{s(x) \mid s \in \Gamma_{0}(\mathcal{B})\right\}$ for each $x \in X$;

- $\Gamma_{0}(\mathcal{B})$ is closed under multiplication by $C_{0}(X)$; and

- For each $s \in \Gamma_{0}(\mathcal{B})$, the function $x \mapsto\|s(x)\|$ is upper semi-continuous, i.e., the set $\{x \in X \mid\|s(x)\|<\epsilon\}$ is open in $X$ for all $\epsilon>0$.

In this paper we will only be concerned with $C^{*}$-bundles that have non-zero fibres.

If $\mathcal{B}$ is a $C^{*}$-bundle over $X$, then its section algebra $\Gamma_{0}(\mathcal{B})$ is a $C_{0}(X)$-algebra: its structure map $\iota$ from $C_{0}(X)$ is defined by $\iota(\mathrm{f}) s=\mathrm{f} s$. Conversely, if $\mathcal{A}$ is a $C_{0}(X)$-algebra, then the fibre $\mathcal{A}_{x}$ of $\mathcal{A}$ over $x \in X$ is $\mathcal{A}_{x}:=\mathcal{A} / \mathcal{I}_{x}$, where $\mathcal{I}_{x}=\left\{\mathrm{f} \cdot a \mid \mathrm{f} \in C_{0}(X), \mathrm{f}(x)=0, a \in \mathcal{A}\right\}$ is 
identified as the ideal in $\mathcal{A}$ of sections vanishing at $x$. If $a \in \mathcal{A}$, we write $a(x)=a+\mathcal{I}_{x}$ for its image in $\mathcal{A}_{x}$. The function $x \mapsto\|a(x)\|$ is upper semi-continuous and vanishes at infinity with

$$
\|a\|=\sup _{x \in X}\|a(x)\|
$$

for all $a \in \mathcal{A}$. The elements $a \in \mathcal{A}$ can in this way be viewed as sections of a $C^{*}$-bundle $\left(\mathcal{A}_{x}\right)_{x \in X}$. We will sometimes use the notation $\coprod_{x \in X} \mathcal{A}_{x}$ for $\mathcal{A}$ when we wish to emphasise its structure as a $C^{*}$-bundle over $X$ with fibre $C^{*}$-algebras $\mathcal{A}_{x}$. These definitions do not require local triviality of the bundle nor the fibres of the bundle to be isomorphic to one another. $C^{*}$-algebra bundles over $X$ are objects of a category whose morphisms are fibrewise $*$-homomorphisms, i.e., $C_{0}(X)$ linear morphisms $\psi: \mathcal{A} \rightarrow \mathcal{B}: \psi(\mathrm{f} \cdot a)=\mathrm{f} \cdot \psi(a)$ for all $\mathrm{f} \in C_{0}(X)$ and $a \in \mathcal{A}$; then $\psi$ induces *-homomorphisms $\psi_{x}: \mathcal{A}_{x} \rightarrow \mathcal{B}_{x}$ such that $\psi_{x}(a(x))=\psi(a)(x)$ for all $a \in \mathcal{A}$.

Example 4.5 (trivial $C^{*}$-algebra bundles). If $\mathcal{D}$ is any $C^{*}$-algebra, then $\mathcal{A}=C_{0}(X, \mathcal{D}) \simeq$ $C_{0}(X) \otimes \mathcal{D}$ is naturally a $C_{0}(X)$-algebra with structure map

$$
(\iota(\mathbf{f}) a)(x):=\mathbf{f}(x) a(x)
$$

for $\mathrm{f} \in C_{0}(X), a \in \mathcal{A}$ and $x \in X$. In this case each fibre $\mathcal{A}_{x}$ is canonically identified with $\mathcal{D}$ and elements of $\mathcal{A}$ are obviously identified with sections.

Example 4.6 (continuous maps). Let $X$ and $Y$ be locally compact spaces and $\sigma: Y \rightarrow X$ a continuous surjective map. Then $C_{0}(Y)$ is a $C_{0}(X)$-algebra with structure map $\iota(\mathrm{f}) \mathrm{g}:=$ $(\mathrm{f} \circ \sigma) \mathrm{g}$, for $\mathrm{f} \in C_{0}(X)$ and $\mathrm{g} \in C_{0}(Y)$, and fibers $C_{0}(Y)_{x} \simeq C_{0}\left(\sigma^{-1}(x)\right)$.

In this paper we are particularly interested in crossed products of $C^{*}$-algebra bundles. Let $\mathcal{A}$ be a $C_{0}(X)$-algebra, and denote by $\operatorname{Aut}_{X}(\mathcal{A})$ the group of fibrewise automorphisms of $\mathcal{A}$. A fibrewise action of a locally compact group $\mathrm{G}$ on $\mathcal{A}$ is then a group homomorphism $\alpha: \mathrm{G} \rightarrow \operatorname{Aut}_{X}(\mathcal{A})$. This implies that $\alpha$ induces an action $\alpha^{x}$ on each fiber $\mathcal{A}_{x}$ for $x \in X$, and in this case we say that the dynamical system $(\mathcal{A}, \mathrm{G}, \alpha)$ is $C_{0}(X)$-linear.

Theorem 4.7. Let $X$ be a locally compact Hausdorff space, and let $(\mathcal{A}, \mathrm{G}, \alpha)$ be a $C_{0}(X)$-linear $C^{*}$-dynamical system. Then the crossed product $\mathcal{A} \rtimes_{\alpha} \mathrm{G}$ is again a $C_{0}(X)$-algebra with fibres

$$
\left(\mathcal{A} \rtimes_{\alpha} \mathrm{G}\right)_{x} \simeq \mathcal{A}_{x} \rtimes_{\alpha^{x}} \mathrm{G}
$$

where

$$
\alpha_{\gamma}^{x}(a(x))=\alpha_{\gamma}(a)(x)
$$

for each $x \in X, \gamma \in \mathrm{G}$ and $a \in \mathcal{A}$.

Proof. The structure map of $\mathcal{A} \rtimes_{\alpha} \mathrm{G}$ is given by precomposing the structure map of $\mathcal{A}$ with the natural injection of the center of the multiplier algebra of $\mathcal{A}$ into the center of the multiplier algebra of $\mathcal{A} \rtimes_{\alpha} \mathrm{G}$; it satisfies $(\mathrm{f} \cdot f)(\gamma)=\mathrm{f} \cdot(f(\gamma))$ for all $\mathrm{f} \in C_{0}(X), f \in C_{\mathrm{c}}(\mathrm{G}, \mathcal{A})$ and $\gamma \in \mathrm{G}$. See [52, Theorem 8.4] for further details.

Example 4.8 (transformation groups). Let $(X, \mathrm{G})$ be a second countable transformation group whose quotient $X / \mathrm{G}$ is a Hausdorff space. By Example 4.6, $C_{0}(X)$ is a $C_{0}(X / \mathrm{G})$-algebra whose fiber over $\mathrm{G} \cdot x$ is isomorphic to $C_{0}\left(\mathrm{G} / \mathrm{G}_{x}\right)$, where $\mathrm{G}_{x}=\{\gamma \in \mathrm{G} \mid \gamma \cdot x=x\}$ is the stabilizer subgroup at $x \in X$. Then the crossed product $C_{0}(X) \rtimes_{\alpha} \mathrm{G}$ is the section algebra of a $C^{*}$-algebra bundle over $X / \mathrm{G}$ whose fiber over $\mathrm{G} \cdot x$ is isomorphic to $C^{*}\left(\mathrm{G}_{x}\right) \otimes \mathcal{K}\left(\mathrm{L}^{2}\left(\mathrm{G} / \mathrm{G}_{x}\right)\right)$ [51]. In the special case where $\mathrm{G}=\mathbb{R}$ and $\mathrm{G}_{x}=\mathbb{Z}$ for all $x \in X$, this is contained in the statement of Proposition 3.9. 
Example 4.9 (principal torus bundles). Let $E \rightarrow X$ be a principal $\mathbb{T}^{r}$-bundle. By Example 4.8, $C_{0}(E)$ is a $C_{0}(X)$-algebra with fibers $C_{0}(E)_{x} \simeq C\left(\mathbb{T}^{r}\right)$, and by (2.10) there is a stable isomorphism $C_{0}(E) \rtimes_{\mathrm{rt}} \mathbb{T}^{r} \simeq C_{0}(X) \otimes \mathcal{K}\left(\mathrm{L}^{2}\left(\mathbb{T}^{r}\right)\right)$. More generally, a noncommutative principal $\mathbb{T}^{r}$-bundle on $X$ is a $C_{0}(X)$-linear $C^{*}$-dynamical system $\left(\mathcal{A}, \mathbb{T}^{r}, \alpha\right)$ with an isomorphism

$$
\mathcal{A} \rtimes_{\alpha} \mathbb{T}^{r} \simeq C_{0}(X, \mathcal{K})
$$

of $C^{*}$-algebra bundles over $X$. For further details and a classification of noncommutative principal torus bundles, see $[15,21]$.

Example 4.10 (noncommutative correspondences). The noncommutative correspondence $\mathcal{C}=$ $C\left(\mathbb{T}_{\Lambda_{\mathrm{G}}}\right) \rtimes_{\mathrm{rt}} \mathbb{Z}^{n}$ from Proposition 3.8 is a noncommutative principal $\mathbb{T}^{n}$-bundle on $X=\mathbb{T}_{\Lambda_{\mathrm{G}}}$ in the sense of Example 4.9: The $C^{*}$-algebra $\mathcal{C}$ is naturally equipped with the dual action of $\mathbb{T}^{n}=\widehat{\mathbb{Z}^{n}}$, and the Takai duality theorem implies that there is an isomorphism $\mathcal{C} \rtimes_{\widehat{\mathrm{rt}}} \mathbb{T}^{n} \simeq$ $C\left(\mathbb{T}_{\Lambda_{\mathrm{G}}}\right) \otimes \mathcal{K}\left(\ell^{2}\left(\mathbb{Z}^{n}\right)\right)$ of $C^{*}$-algebra bundles over the twisted torus $\mathbb{T}_{\Lambda_{\mathrm{G}}}$.

Remark 4.11. There is a natural notion of Morita equivalence of $C^{*}$-algebra bundles over $X$, similar to the notion of equivariant Morita equivalence from Theorem 2.12, which uses the $C_{0}(X)$-bimodule structures: a $C_{0}(X)$-linear Morita equivalence between two $C_{0}(X)$-algebras is a Morita equivalence which is compatible with the $C^{*}$-bundle structures over $X$. More generally, there is a category $\mathcal{R} \mathscr{K}_{X}$ of $C^{*}$-algebra bundles over $X$ whose morphisms are elements of Kasparov's groups $\mathcal{R} K K(X ; \mathcal{A}, \mathcal{B})$, see, e.g., [15]: the cycles are the usual cycles $(\mathcal{H}, \phi, T)$ for Kasparov's bivariant K-theory $\operatorname{KK}(\mathcal{A}, \mathcal{B})$ (cf. Section 3.2) with the additional requirement that $\phi: \mathcal{A} \rightarrow \operatorname{End}_{\mathcal{B}}(\mathcal{H})$ is $C_{0}(X)$-linear. There is an obvious faithful functor $\mathcal{R} \mathscr{K}_{X} \rightarrow \mathscr{K} \mathscr{K}$ which forgets the $C_{0}(X)$-algebra structures. Isomorphic $C^{*}$-bundles in the category $\mathcal{R} \mathscr{K}_{X}$ are precisely the $\mathcal{R} K K$-equivalent $C^{*}$-bundles. If $\mathcal{A}$ and $\mathcal{B}$ are isomorphic in $\mathcal{R} \mathscr{K} \mathscr{K}_{X}$, i.e., there exists an invertible class $\alpha \in \mathcal{R} \operatorname{KK}(X ; \mathcal{A}, \mathcal{B})$, then they are also isomorphic as $C^{*}$-algebras in the category $\mathscr{K} \mathscr{K}$.

\section{$4.4 \quad \mathbb{R}^{n}$-actions on Mostow bundles}

We can now apply the results of Section 3 to the class of twisted tori given in Section 4.2. The Mostow fibration of any almost abelian solvmanifold identifies $\mathbb{T}_{\Lambda_{G}}$ as a torus bundle over a circle, hence the algebra of functions $C\left(\mathbb{T}_{\Lambda_{\mathrm{G}}}\right)$ is a $C(\mathbb{T})$-algebra. In other words, $C\left(\mathbb{T}_{\Lambda_{\mathrm{G}}}\right)$ is an object of the category $\mathcal{R} \mathscr{K} \mathscr{K}_{\mathbb{T}}$, and we are interested in the T-duality isomorphisms of $C\left(\mathbb{T}_{\Lambda_{\mathrm{G}}}\right)$ in this category. In particular, given a fibrewise right action of the abelian Lie group $\mathbb{R}^{n}$ on $\mathbb{T}_{\Lambda_{G}}$, it follows from Theorem 4.7 that the $C^{*}$-algebraic T-dual $C\left(\mathbb{T}_{\Lambda_{\mathrm{G}}}\right) \rtimes_{\mathrm{rt}} \mathbb{R}^{n}$ is also a $C(\mathbb{T})$-algebra, and by [15, Theorem 3.5] the $C^{*}$-bundles $C\left(\mathbb{T}_{\Lambda_{\mathrm{G}}}\right)$ and $C\left(\mathbb{T}_{\Lambda_{\mathrm{G}}}\right) \rtimes_{\mathrm{rt}} \mathbb{R}^{n}$ are isomorphic as $C^{*}$-algebras in the category $\mathcal{R} \mathscr{K} \mathscr{K}_{\mathbb{T}}$.

In order to have sensible definitions of T-duality, we need to identify the homologically nontrivial one-cycles of the twisted torus $\mathbb{T}_{\Lambda_{G}}$, which are determined in [2, Proposition 4.7]. Write

$$
\mathbf{M}=\left(m_{i j}\right)
$$

for the integer matrix elements $m_{i j} \in \mathbb{Z}$ of the monodromy matrix. Since $G=\mathbb{R}^{d-1} \rtimes_{\varphi} \mathbb{R}$ is simply-connected, the fundamental group of the twisted torus is $\pi_{1}\left(\mathbb{T}_{\Lambda_{G}}\right) \simeq \Lambda_{G}$ whose abelianisation $\Lambda_{\mathrm{G}} /\left[\Lambda_{\mathrm{G}}, \Lambda_{\mathrm{G}}\right]$ gives the first homology group via the presentation

$$
\left.\mathrm{H}_{1}\left(\mathbb{T}_{\Lambda_{G}}, \mathbb{Z}\right)=\mathbb{Z} \oplus\left\langle\hat{e}_{1}, \ldots, \hat{e}_{d-1}\right| \sum_{j=1}^{d-1} m_{j i} \hat{e}_{j}=\hat{e}_{i} \text { for } i=1, \ldots, d-1\right\rangle,
$$


where the first factor of $\mathbb{Z}$ corresponds to the base circle of the torus fibration and the generators $\hat{e}_{1}, \ldots, \hat{e}_{d-1}$ correspond to the torus fibres; they are given by

$$
\hat{e}_{i}:=\sum_{k=1}^{d-1} \Sigma_{k i} \vec{e}_{k},
$$

where $\vec{e}_{1}, \ldots, \vec{e}_{d-1}$ is the standard basis of $\mathbb{Z}^{d-1}$ giving the group law (4.3).

We can then apply the structure theorem for finitely-generated $\mathbb{Z}$-modules by appealing to some classical matrix algebra. From the presentation $(4.5), \mathrm{H}_{1}\left(\mathbb{T}_{\Lambda_{G}}, \mathbb{Z}\right)=\mathbb{Z} \oplus \operatorname{coker}\left(\varphi_{x_{0}}-\mathrm{id}_{\mathbb{Z}^{d-1}}\right)$ where $\left(\varphi_{x_{0}}-\mathrm{id}_{\mathbb{Z}^{d-1}}\right): \mathbb{Z}^{d-1} \rightarrow \mathbb{Z}^{d-1}$ in the basis $\hat{e}_{1}, \ldots, \hat{e}_{d-1}$ is given by the integer relation matrix

$$
\mathrm{A}:=\mathrm{M}-\mathbb{1}_{d-1} .
$$

Let $r$ be the rank of A. This matrix can be brought into its Smith normal form $\mathrm{D}$ by finding invertible integer matrices $\mathrm{L}, \mathrm{R} \in \mathrm{GL}(d-1, \mathbb{Z})$ such that

$$
\mathrm{D}=\mathrm{LAR}
$$

is diagonal with entries $m_{i} \in \mathbb{Z}$ for $i=1, \ldots, d-1$. The integers $m_{i}$ are the elementary divisors of A. They have the properties that $m_{i}$ divides $m_{i+1}$, for $0<i<d-1$, and in particular $m_{i}=0$ for $i>r$; they can be computed explicitly (up to sign) as

$$
m_{i}=\frac{d_{i}(\mathrm{~A})}{d_{i-1}(\mathrm{~A})},
$$

where the $i$-th determinant divisor $d_{i}(\mathrm{~A})$ is the greatest common divisor of all $i \times i$ minors of the relation matrix $A$, with $d_{0}(\mathrm{~A}):=1$. The matrices $\mathrm{L}, \mathrm{R} \in \mathrm{GL}(d-1, \mathbb{Z})$ are found by reducing the matrix A to its Smith normal form D through a sequence of elementary row and column operations over $\mathbb{Z}$, see, e.g., [23].

Given the Smith normal form, we set

$$
\tilde{e}_{i}:=\sum_{j=1}^{d-1}\left(\mathrm{~L}^{-1}\right)_{j i} \hat{e}_{j}
$$

and observe that the image of $\mathrm{A}$, which is generated over $\mathbb{Z}$ by the vectors

$$
\sum_{j=1}^{d-1} \mathrm{~A}_{j i} \hat{e}_{j}=\sum_{k, l=1}^{d-1}\left(\mathrm{R}^{-1}\right)_{k i} \mathrm{D}_{l k} \tilde{e}_{k}
$$

is equivalently generated by the vectors $m_{k} \tilde{e}_{k}$ with $k=1, \ldots, r$. Hence

$$
\begin{aligned}
\operatorname{coker}\left(\varphi_{x_{0}}-\operatorname{id}_{\mathbb{Z}^{d-1}}\right) & =\left\langle\hat{e}_{1}, \ldots, \hat{e}_{d-1}\right\rangle /\left\langle\sum_{j=1}^{d-1} \mathrm{~A}_{j 1} \hat{e}_{j}, \ldots, \sum_{j=1}^{d-1} \mathrm{~A}_{j d-1} \hat{e}_{j}\right\rangle \\
& =\left\langle\tilde{e}_{1}, \ldots, \tilde{e}_{d-1}\right\rangle /\left\langle m_{1} \tilde{e}_{1}, \ldots, m_{r} \tilde{e}_{r}\right\rangle
\end{aligned}
$$

and

$$
\mathrm{H}_{1}\left(\mathbb{T}_{\Lambda_{\mathrm{G}}}, \mathbb{Z}\right) \simeq \mathbb{Z} \oplus \mathbb{Z}^{d-1-r} \oplus \bigoplus_{i=1}^{r} \mathbb{Z}_{m_{i}} .
$$

We are exclusively interested in the natural $\mathbb{R}^{n}$-actions on $\mathbb{T}_{\Lambda_{\mathrm{G}}}$ which descend from actions of abelian subgroups $\mathbb{R}^{n} \subset \mathrm{G}$, acting on $\mathrm{G}$ by right multiplication. They can be organised into 
three classes associated with the different types of summands in the $\mathbb{Z}$-module presentation of the homology group (4.9), and we only retain those which are fiberwise actions on the Mostow bundle. The first summand $\mathbb{Z}$ in (4.9) corresponds to the subgroup

$$
\mathbb{R}_{x}=\{(0, \xi) \in \mathrm{G}\}
$$

acting on $\mathrm{G}$ by right multiplication:

$$
(z, x)(0, \xi)=(z, x+\xi)
$$

for all $(z, x) \in \mathrm{G}$ and $\xi \in \mathbb{R}$. Clearly this does not descend to a fiberwise action on the twisted torus $\mathbb{T}_{\Lambda_{\mathrm{G}}}$, and the crossed product $C\left(\mathbb{T}_{\Lambda_{\mathrm{G}}}\right) \rtimes_{\mathrm{rt}} \mathbb{R}_{x}$ is no longer a $C(\mathbb{T})$-algebra. Thus an $\mathbb{R}_{x^{-}}$ action takes us out of the category $\mathcal{R} \mathscr{K}_{\mathscr{T}}$, and we will henceforth discard $\mathbb{R}^{n}$-actions where $\mathbb{R}^{n}$ contains the subgroup $\mathbb{R}_{x}$.

For the remaining types of summands in (4.9), we can give explicit descriptions of the $C^{*}$ algebraic T-duals of an almost abelian solvmanifold. We consider both classes in turn. As the only solvmanifolds in one and two dimensions are tori, which are already treated by our analysis from Section 3.4, we assume $d \geq 3$ for the remainder of this paper.

\section{5 $\mathbb{R}_{y}$-actions: Circle bundles with $H$-flux}

Let us consider the second summand $\mathbb{Z}^{d-1-r}$ in (4.9), which corresponds to the lattice $\Lambda_{\mathrm{G}} \cap$ $\operatorname{ker}\left(\varphi_{x_{0}}-\mathrm{id}_{\mathbb{R}^{d-1}}\right)$ in $\operatorname{ker}\left(\varphi_{x_{0}}-\mathrm{id}_{\mathbb{R}^{d-1}}\right)$. In terms of the generators

$$
e_{i}^{\prime}:=\sum_{j=1}^{d-1} \mathrm{R}_{j i} \hat{e}_{j}
$$

of $\Lambda_{\mathrm{G}} \cap \mathrm{N}$, the sublattice $\Lambda_{\mathrm{G}} \cap \operatorname{ker}\left(\varphi_{x_{0}}-\mathrm{id}_{\mathbb{R}^{d-1}}\right)$ is generated by the vectors $e_{r+1}^{\prime}, e_{r+2}^{\prime}, \ldots, e_{d-1}^{\prime}$ in the kernel of the relation matrix (4.7). We begin with some elementary observations. Firstly, the subgroups $\operatorname{ker}\left(\varphi_{x_{0}}-\mathrm{id}_{\mathbb{R}^{d-1}}\right)$ and $\Lambda_{\mathrm{G}}$ commute in $\mathrm{G}:(-v, 0)\left(0, x_{0}\right)(v, 0)=\left(0, x_{0}\right) \in \Lambda_{\mathrm{G}}$ for all $v \in \operatorname{ker}\left(\varphi_{x_{0}}-\mathrm{id}_{\mathbb{R}^{d-1}}\right)$. Secondly, $\operatorname{ker}\left(\varphi_{x_{0}}-\mathrm{id}_{\mathbb{R}^{d-1}}\right)$ is a closed abelian normal subgroup of $\mathrm{G}$ : $(z, x)(v, 0)(z, x)^{-1}=\left(\varphi_{x}(v), 0\right) \in \operatorname{ker}\left(\varphi_{x_{0}}-\operatorname{id}_{\mathbb{R}^{d-1}}\right)$ since $\varphi_{x_{0}}\left(\varphi_{x}(v)\right)=\varphi_{x}\left(\varphi_{x_{0}}(v)\right)=\varphi_{x}(v)$.

In the following we consider a subgroup $\mathrm{V} \simeq \mathbb{R}^{n} \subset \operatorname{ker}\left(\varphi_{x_{0}}-\mathrm{id}_{\mathbb{R}^{d-1}}\right)$ which is normal in $\mathrm{G}$ such that $\mathrm{V}^{\mathbb{Z}}:=\mathrm{V} \cap \Lambda_{\mathrm{G}}$ is a lattice in $\mathrm{V}$. This is the case, for example, if $\mathrm{V}$ is the span of a subset of the generators $e_{r+1}^{\prime}, e_{r+2}^{\prime}, \ldots, e_{d-1}^{\prime}$. As an immediate consequence of Lemma 2.3 we then have

Proposition 4.12. The quotient group $\mathrm{G}^{\mathrm{V}}:=\mathrm{G} / \mathrm{V}$ is a $(d-n)$-dimensional almost abelian solvable Lie group

$$
\mathrm{G}^{\vee} \simeq \mathbb{R}^{d-n-1} \rtimes_{\varphi} \vee \mathbb{R}
$$

where $\varphi^{\vee}: \mathbb{R} \rightarrow \mathrm{GL}(d-n-1, \mathbb{R})$ is defined by $\varphi_{x}^{\vee}[z]=\left(\mathbb{1}_{d-1}-\operatorname{pr}_{\vee}\right) \varphi_{x}(z)$ for all $x \in \mathbb{R}$ and $z \in \mathbb{R}^{d-1}$, with $\mathrm{pr}_{\mathrm{\vee}}$ the projection of $\mathbb{R}^{d-1}$ to $\mathrm{V}$ and $[z]=\left(\mathbb{1}_{d-1}-\mathrm{pr}_{\mathrm{V}}\right)(z) \in \mathbb{R}^{d-1} / \mathrm{V}$.

We are particularly interested in the induced action of $\mathrm{V}$ on the twisted torus $\mathbb{T}_{\Lambda_{\mathrm{G}}}$.

Proposition 4.13. Let $\mathrm{V}$, as above, be normal in $\mathrm{G}$ and let $\Lambda_{\mathrm{G}}$ be normal in $\Lambda_{\mathrm{G}} \mathrm{V}$. Then the quotient map $p_{\mathrm{V}}: \mathbb{T}_{\Lambda_{\mathrm{G}}} \rightarrow \mathbb{T}_{\Lambda_{\mathrm{G}}} / \mathrm{V}$ is a principal torus bundle of rank $n=\operatorname{dim} \mathrm{V}$ over an almost abelian solvmanifold $\mathbb{T}_{\Lambda_{\mathrm{G}} \mathrm{V}}$ of dimension $d-n$. Its Chern class $c_{1}\left(p_{\mathrm{V}}\right) \in \mathrm{H}^{2}\left(\mathbb{T}_{\Lambda_{\mathrm{G}} \mathrm{V}}, \mathbb{Z}\right)$ can be computed by Chern-Weil theory from the curvature of the connection $\kappa_{\mathrm{V}} \in \Omega^{1}\left(\mathbb{T}_{\Lambda_{\mathrm{G}}}, \mathrm{V}\right)$ given by

$$
\kappa_{\mathrm{V}}=-\left(\operatorname{pr}_{\mathrm{V}} \varphi_{-x}\right) \cdot \mathrm{d} z
$$

in the notation of Proposition 4.12 . 
Proof. The first statement follows from Proposition 4.3. For the Chern class, we note that the left-invariant Maurer-Cartan one-forms on the Lie group $\mathrm{G}$ are given by $\mathrm{d} x$ and $P \in \Omega^{1}\left(\mathrm{G}, \mathbb{R}^{d-1}\right)$ where

$$
P=\varphi_{-x} \cdot \mathrm{d} z
$$

and this descends to the twisted torus $\mathbb{T}_{\Lambda_{G}}$. The desired principal $\mathbb{T}^{n}$-connection on $\mathbb{T}_{\Lambda_{\mathrm{G}}}$ is then given by

$$
\kappa_{\mathrm{V}}=-\operatorname{pr}_{\mathrm{V}} P=-\left(\operatorname{pr}_{\mathrm{V}} \varphi_{-x}\right) \cdot \mathrm{d} z
$$

and the result follows.

By virtue of the fibration $p_{\mathrm{V}}: \mathbb{T}_{\Lambda_{\mathrm{G}}} \rightarrow \mathbb{T}_{\Lambda_{\mathrm{G}} \mathrm{V}}$, the algebra of functions $C\left(\mathbb{T}_{\Lambda_{\mathrm{G}}}\right)$ is also a $C\left(\mathbb{T}_{\Lambda_{\mathrm{GV}}}\right)$ algebra. We are particularly interested in the case $n=1$, whereby we can explicitly apply our framework of topological T-duality. Combining Propositions 4.13 and 3.9, we immediately arrive at

Theorem 4.14. Let $y_{0} \in \operatorname{ker}\left(\varphi_{x_{0}}-\mathrm{id}_{\mathbb{R}^{d-1}}\right)$ and let

$$
\mathbb{R}_{y_{0}}:=\mathbb{R}\left(y_{0}, 0\right)
$$

be the corresponding one-dimensional subgroup. Suppose that $\mathbb{R}_{y_{0}}$ is normal in $\mathrm{G}$ and $\Lambda_{\mathrm{G}}$ is normal in $\Lambda_{\mathrm{G}} \mathbb{R}_{y_{0}}$ (this is the case, for example, if $\left(y_{0}, 0\right)$ is in the center of $\mathrm{G}$ ). Let $\mathrm{G}^{y_{0}}=\mathrm{G} / \mathbb{R}_{y_{0}}$ be the almost abelian solvable Lie group constructed by Proposition 4.12 , and $p_{y_{0}}: \mathbb{T}_{\Lambda_{\mathrm{G}}} \rightarrow \mathbb{T}_{\Lambda_{\mathrm{G}} y_{0}}$ the principal circle bundle constructed by Proposition 4.13. Then the $C^{*}$-algebraic T-dual

$$
C\left(\mathbb{T}_{\Lambda_{\mathrm{G}}}\right) \rtimes_{\mathrm{rt}} \mathbb{R}_{y_{0}} \simeq \mathrm{CT}\left(\mathbb{T}_{\Lambda_{\mathrm{G}} y_{0}} \times \mathbb{T}_{y_{0}}, \delta_{y_{0}}\right)
$$

is a continuous-trace algebra with spectrum $\mathbb{T}_{\Lambda_{\mathrm{G}} y_{0}} \times \mathbb{T}_{y_{0}}$ and Dixmier-Douady class

$$
\delta_{y_{0}}=c_{1}\left(p_{y_{0}}\right) \smile \zeta_{y_{0}},
$$

where $\zeta_{y_{0}}$ is the standard generator of $\mathrm{H}^{1}\left(\mathbb{T}_{y_{0}}, \mathbb{Z}\right) \simeq \mathbb{Z}$ and the Chern-Weil representative of $c_{1}\left(p_{y_{0}}\right) \in \mathrm{H}^{2}\left(\mathbb{T}_{\Lambda_{\mathrm{G}} y_{0}}, \mathbb{Z}\right)$ is the curvature of the connection $\kappa_{y_{0}} \in \Omega^{1}\left(\mathbb{T}_{\Lambda_{\mathrm{G}}}\right)$ on this circle bundle given by

$$
\kappa_{y_{0}}=-\left(\operatorname{pr}_{y_{0}} \varphi_{-x}\right) \cdot \mathrm{d} z .
$$

Thus in the case of an action of $\mathbb{R}$ due to a normal subgroup of $G$ that is in $\operatorname{ker}\left(\varphi_{x_{0}}-\mathrm{id}_{\mathbb{R}^{d-1}}\right)$ in the setting of Theorem 4.14, which we collectively refer to as $\mathbb{R}_{y}$-actions, the T-dual of an almost abelian solvmanifold $\mathbb{T}_{\Lambda_{\mathrm{G}}}$ is the Hausdorff space $X=\mathbb{T}_{\Lambda_{\mathrm{G}} y_{0}} \times \mathbb{T}_{y_{0}}$ with a three-form ' $H$-flux' whose cohomology class is represented by $\left[H_{y_{0}}\right]=c_{1}\left(p_{y_{0}}\right) \smile \zeta_{y_{0}}$. The associated correspondence space construction proceeds analogously to Section 3.5, which we can give explicitly as

Proposition 4.15. The topological T-duality of Theorem 4.14 is implemented by the noncommutative correspondence

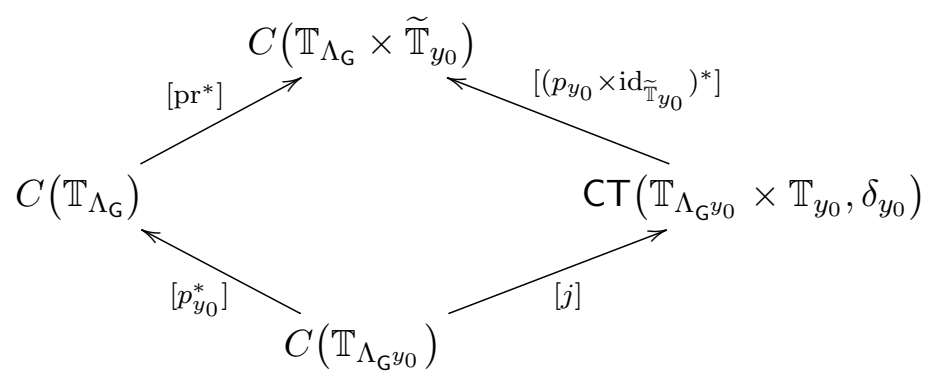

as a diagram in the category $\mathcal{R} \mathscr{K}_{\mathbb{T}}$. 
Proof. Since the subgroup

$$
\mathbb{Z}_{y_{0}}:=\mathbb{R}_{y_{0}} \cap \Lambda_{\mathrm{G}}
$$

acts trivially on the algebra of functions $C\left(\mathbb{T}_{\Lambda_{\mathrm{G}}}\right)$, there is an isomorphism

$$
C\left(\mathbb{T}_{\Lambda_{\mathrm{G}}}\right) \rtimes_{\mathrm{rt}} \mathbb{Z}_{y_{0}} \simeq C\left(\mathbb{T}_{\Lambda_{\mathrm{G}}} \times \widetilde{\mathbb{T}}_{y_{0}}\right),
$$

where $\widetilde{\mathbb{T}}_{y_{0}}$ is the circle dual to $\mathbb{T}_{y_{0}} \simeq \mathbb{R}_{y_{0}} / \mathbb{Z}_{y_{0}}$. Proposition 4.13 shows that the Chern-Weil representative of $c_{1}\left(p_{y_{0}}\right) \in \mathrm{H}^{2}\left(\mathbb{T}_{\Lambda_{\mathrm{G}} y_{0}}, \mathbb{Z}\right)$ pulls back to the exact two-form $\mathrm{d} \kappa_{y_{0}} \in \Omega^{2}\left(\mathbb{T}_{\Lambda_{\mathrm{G}}}\right)$ under the bundle projection $p_{y_{0}}: \mathbb{T}_{\Lambda_{\mathrm{G}}} \rightarrow \mathbb{T}_{\Lambda_{\mathrm{G}}^{y_{0}}}$. Hence $p_{y_{0}}^{*} c_{1}\left(p_{y_{0}}\right)=0$ which implies

$$
\left(p_{y_{0}} \times \mathrm{id}_{\widetilde{\mathbb{T}}_{y_{0}}}\right)^{*}\left(c_{1}\left(p_{y_{0}}\right) \smile \zeta_{y_{0}}\right)=0 \in \mathrm{H}^{3}\left(\mathbb{T}_{\Lambda_{\mathrm{G}}} \times \widetilde{\mathbb{T}}_{y_{0}}, \mathbb{Z}\right)
$$

Thus the algebra $\mathrm{CT}\left(\mathbb{T}_{\Lambda_{\mathrm{G}}^{y_{0}}} \times \mathbb{T}_{y_{0}}, \delta_{y_{0}}\right)$ pulls back to an algebra isomorphic to $C\left(\mathbb{T}_{\Lambda_{\mathrm{G}}} \times \widetilde{\mathbb{T}}_{y_{0}}\right) \otimes \mathcal{K}$ by $p_{y_{0}} \times \mathrm{id}_{\widetilde{\mathbb{T}}_{y_{0}}}$, and the result follows.

\section{6 $\mathbb{R}_{z}$-actions: noncommutative torus bundles}

Let us now come to the torsion summands $\mathbb{Z}_{m_{i}}$ in the $\mathbb{Z}$-module decomposition (4.9). Pick a non-trivial elementary divisor $m_{i}>1$ for some $i \in\{1, \ldots, r\}$. The corresponding homology generator $\tilde{e}_{i}$ is constructed as a $\mathbb{Z}$-linear combination (4.8) of the generators (4.6). It defines a fixed element $z_{0}$ in the image of $\varphi_{x_{0}}-\mathrm{id}_{\mathbb{R}^{\mathrm{d}-1}}$ and a corresponding one-dimensional subgroup of $\mathrm{G}$ given by

$$
\mathbb{R}_{z_{0}}:=\mathbb{R}\left(z_{0}, 0\right) .
$$

We further assume that

$$
\mathbb{Z}_{z_{0}}:=\mathbb{R}_{z_{0}} \cap \Lambda_{\mathrm{G}}
$$

is a lattice in $\mathbb{R}_{z_{0}}$. The choice of $z_{0} \in \mathbb{R}^{d-1}$ is not unique, and any change of basis of $\mathbb{Z}^{d-1}$, represented by a matrix $B \in \mathrm{GL}(d-1, \mathbb{Z})$, defines an equally good element $B \cdot z_{0} \in \mathbb{R}^{d-1}$ as long as $B \cdot z_{0} \in \operatorname{im}\left(\varphi_{x_{0}}-\mathrm{id}_{\mathbb{R}^{d-1}}\right)$. We write $\mathrm{pr}_{z_{0}}$ for the linear projection of $\mathbb{R}^{d-1}$ to $\mathbb{R}_{z_{0}}$, and denote by $\left\langle z_{0}, z\right\rangle \in \mathbb{R}$ the component of $z \in \mathbb{R}^{d-1}$ in $\mathbb{R}_{z_{0}}$, i.e., $\operatorname{pr}_{z_{0}} \cdot z=\left\langle z_{0}, z\right\rangle z_{0}$.

In the basis $\hat{e}_{i}$, the lattice of $\mathrm{G}$ is given by

$$
\Lambda_{\mathrm{G}}=\mathbb{Z}^{d-1} \rtimes_{\left.\hat{\varphi}\right|_{x_{0}} \mathbb{Z}} x_{0} \mathbb{Z}
$$

where $\hat{\varphi}_{x}:=\Sigma^{-1} \varphi_{x} \Sigma$ for all $x \in \mathbb{R}$. Then the fibres of the underlying Mostow bundle are 'square' tori $\mathbb{T}^{d-1}$ with unit periodicities $z \sim z+\vec{e}_{i}$ for $i=1, \ldots, d-1$, where as before $\vec{e}_{i}$ denotes the standard basis of $\mathbb{R}^{d-1}$. The action of the subgroup $\mathbb{R}_{z_{0}}$ on elements $(z, x) \in \mathrm{G}$ by right multiplication is given by

$$
(z, x)\left(\zeta z_{0}, 0\right)=\left(z+\zeta \Sigma^{-1} \varphi_{x} \cdot z_{0}, x\right)
$$

for $\zeta \in \mathbb{R}$, where $\Sigma^{-1} \varphi_{x} \cdot z_{0}$ lies in the image of the relation matrix $\mathrm{A}=\mathrm{M}-\mathbb{1}_{d-1}$.

Our principal tool to compute the $C^{*}$-algebraic T-dual for such an action of $\mathbb{R}$ in the image of $\varphi_{x_{0}}-\mathrm{id}_{\mathbb{R}^{d-1}}$, which we collectively refer to as $\mathbb{R}_{z}$-actions, will be Green's theorem in the form (3.3):

$$
C\left(\mathbb{T}_{\Lambda_{\mathrm{G}}}\right) \rtimes_{\mathrm{rt}} \mathbb{R}_{z_{0}} \sim_{\mathrm{M}} C_{0}\left(\mathrm{G} / \mathbb{R}_{z_{0}}\right) \rtimes_{\mathrm{lt}} \Lambda_{\mathrm{G}}
$$


By appealing to Theorem 4.7, we may apply (4.11) fibrewise. For fixed $x \in \mathbb{R}$, the fibre $\mathrm{G}_{x}$ of the semi-direct product $G=\mathbb{R}^{d-1} \rtimes_{\hat{\varphi}} \mathbb{R}$ is the subgroup $\mathbb{R}^{d-1}$, and the corresponding fibre of the solvmanifold $\mathbb{T}_{\Lambda_{G}}$ over $x \in \mathbb{R} / x_{0} \mathbb{Z}$ is the torus $\mathbb{T}^{d-1}=\mathbb{R}^{d-1} / \mathbb{Z}^{d-1}$. The Morita equivalence (4.11) is $C(\mathbb{T})$-linear and the fibres of the corresponding T-dual $C(\mathbb{T})$-algebra are given by the fibrewise Morita equivalence

$$
\left(C\left(\mathbb{T}_{\Lambda_{\mathrm{G}}}\right) \rtimes_{\mathrm{rt}} \mathbb{R}_{z_{0}}\right)_{x} \simeq C\left(\mathbb{R}^{d-1} / \mathbb{Z}^{d-1}\right) \rtimes_{\mathrm{rt}} \mathbb{R}_{z_{0}} \sim_{\mathrm{M}} C_{0}\left(\mathbb{R}^{d-1} / \mathbb{R}_{z_{0}}\right) \rtimes_{\mathrm{lt}^{x}} \mathbb{Z}^{d-1} .
$$

The action of the subgroup $\mathbb{Z}^{d-1} \subset \Lambda_{\mathrm{G}}$ on the coset space $\mathbb{R}^{d-1} / \mathbb{R}_{z_{0}}$ is induced by left multiplication in the group $G$. After a basis transformation, we can decompose the discrete group $\mathbb{Z}^{d-2}$ into a direct sum

$$
\mathbb{Z}^{d-1} \simeq \mathbb{Z}_{v}^{d-2} \oplus \mathbb{Z}_{z_{0}}
$$

where $\mathbb{Z}_{v}^{d-1}=\left(\mathbb{1}_{d-1}-\mathrm{pr}_{z_{0}}\right) \cdot \mathbb{Z}^{d-1}$.

Let

$$
\mathbb{F}_{*}:=\left\{x \in \mathbb{R} \mid\left\langle z_{0}, \Sigma^{-1} \varphi_{x} \cdot z_{0}\right\rangle=0\right\} .
$$

Lemma 4.16. Over any $x \in \mathbb{F}_{*}$, the fiber $\left(C\left(\mathbb{T}_{\Lambda_{\mathrm{G}}}\right) \rtimes_{\mathrm{rt}} \mathbb{R}_{z_{0}}\right)_{x}$ is Morita equivalent to the commutative $C^{*}$-algebra $C\left(\mathbb{T}^{d-1}\right)$.

Proof. If $x \in \mathbb{F}_{*}$, then $w_{0}:=\Sigma^{-1} \varphi_{x} \cdot z_{0}$ only shifts the corresponding component of $z$ in

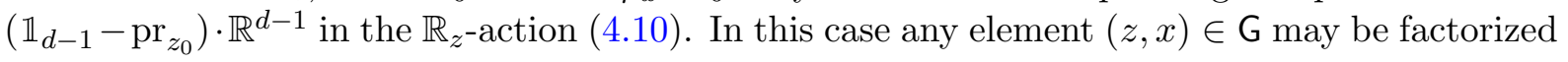
as

$$
(z, x)=\left(\operatorname{pr}_{z_{0}} \cdot z+\left(\mathbb{1}_{d-1}-\operatorname{pr}_{z_{0}}-\operatorname{pr}_{w_{0}}\right) \cdot z, x\right)\left(\left\langle w_{0}, z\right\rangle z_{0}, 0\right) .
$$

Hence the coset space $\mathbb{R}^{d-1} / \mathbb{R}_{z_{0}} \simeq \mathbb{R}^{d-2}$ can be parameterized by $\mathrm{pr}_{z_{0}} \cdot z+\left(\mathbb{1}_{d-1}-\mathrm{pr}_{z_{0}}-\mathrm{pr}_{w_{0}}\right) \cdot z$ with $z \in \mathbb{R}^{d-1}$, which we decompose correspondingly into a direct product $\mathbb{R}_{z_{0}} \times \mathbb{R}^{d-3}$ (with the second factor absent for $d=3$ ). By (4.4) the discrete group $\mathbb{Z}_{v}^{d-2}$ acts trivially on the line $\mathbb{R}_{z_{0}}$ and by translations on $\mathbb{R}^{d-3}$, while $\mathbb{Z}_{z}$ acts by translations on $\mathbb{R}_{z_{0}}$ and trivially on $\mathbb{R}^{d-3}$. Then the crossed product on the right-hand side of (4.12) may be unravelled to get

$$
\begin{aligned}
& C_{0}\left(\mathbb{R}^{d-1} / \mathbb{R}_{z_{0}}\right) \rtimes_{1 \mathrm{t}^{x}} \mathbb{Z}^{d-1} \simeq C_{0}\left(\mathbb{R}_{z_{0}} \times \mathbb{R}^{d-3}\right) \rtimes_{1 \mathrm{lt}^{x}}\left(\mathbb{Z}_{v}^{d-2} \times \mathbb{Z}_{z_{0}}\right) \\
& \simeq\left[\left(C_{0}\left(\mathbb{R}_{z_{0}}\right) \otimes C_{0}\left(\mathbb{R}^{d-3}\right)\right) \rtimes_{\mathrm{id} \otimes \mathrm{lt}^{x}} \mathbb{Z}_{v}^{d-2}\right] \rtimes_{\beta^{x}} \mathbb{Z}_{z_{0}} \\
& \sim_{\mathrm{M}}\left(C_{0}\left(\mathbb{R}_{z_{0}}\right) \otimes C^{*}(\mathbb{Z}) \otimes C\left(\mathbb{T}^{d-3}\right)\right) \rtimes_{1 t^{x} \otimes \mathrm{id} \otimes \mathrm{id}} \mathbb{Z}_{z_{0}} \\
& \simeq\left(C_{0}\left(\mathbb{R}_{z_{0}}\right) \rtimes_{1 \mathrm{t}^{x}} \mathbb{Z}_{z_{0}}\right) \otimes C^{*}(\mathbb{Z}) \otimes C\left(\mathbb{T}^{d-3}\right) \\
& \sim_{\mathrm{M}} C(\mathbb{T}) \otimes C^{*}(\mathbb{Z}) \otimes C\left(\mathbb{T}^{d-3}\right) \simeq C\left(\mathbb{T}^{d-1}\right) .
\end{aligned}
$$

In the second line we applied Theorem 2.6. In the third line we used Example 2.14 together with the fact that the homomorphism $\sigma_{\mathbb{Z}_{z_{0}}}$ from (2.5) is trivial since the groups $\mathbb{Z}_{z_{0}}$ and $\mathbb{Z}_{v}^{d-2}$ are discrete, and so the action of $\mathbb{Z}_{z_{0}}$ on the crossed product is induced by left multiplication on $\mathbb{R}_{z_{0}}$, the trivial action on $\mathbb{R}^{d-3}$, and the trivial action on $\mathbb{Z}_{v}^{d-2}$. In the fifth line we used Example 2.14 again.

The central result of this paper is

Theorem 4.17. The $C^{*}$-algebraic T-dual of any almost abelian solvmanifold $\mathbb{T}_{\Lambda_{\mathrm{G}}}$ with respect to an $\mathbb{R}_{z}$-action is Morita equivalent to a $C^{*}$-algebra bundle of noncommutative tori over the circle $\mathbb{T}$ :

$$
C\left(\mathbb{T}_{\Lambda_{\mathrm{G}}}\right) \rtimes_{\mathrm{rt}} \mathbb{R}_{z_{0}} \sim_{M} \coprod_{x \in \mathbb{R} / \mathbb{Z}} \mathbb{T}_{\vec{\theta}_{z_{0}}(x)}^{d-1},
$$


where the noncommutativity parameters $\vec{\theta}_{z_{0}}(x) \in \mathbb{R}^{d-2}$ are given by

$$
\vec{\theta}_{z_{0}}(x)= \begin{cases}0 & \text { for } x \in \mathbb{F}_{*}, \\ \frac{\left(\mathbb{1}_{d-1}-\mathrm{pr}_{z_{0}}\right) \Sigma^{-1} \varphi_{x_{0} x} \cdot z_{0}}{\left\langle z_{0}, \Sigma^{-1} \varphi_{x_{0} x} \cdot z_{0}\right\rangle} & \text { for } x \in \mathbb{R} \backslash \mathbb{F}_{*} .\end{cases}
$$

Proof. That the fibres over $x \in \mathbb{F}_{*}$ are just ordinary tori $\mathbb{T}^{d-1}$ is established by Lemma 4.16 , so we may assume that $x \in \mathbb{R} \backslash \mathbb{F}_{*}$. Then a simple calculation shows that any element $(z, x) \in \mathrm{G}$ can be factorized as

$$
(z, x)=(v, x)\left(\frac{\mathrm{pr}_{z_{0}} \cdot z}{\left\langle z_{0}, \Sigma^{-1} \varphi_{x} \cdot z_{0}\right\rangle}, 0\right),
$$

where

$$
v:=\left(\mathbb{1}_{d-1}-\mathrm{pr}_{z_{0}}\right) \cdot z-\frac{\left\langle z_{0}, z\right\rangle}{\left\langle z_{0}, \Sigma^{-1} \varphi_{x} \cdot z_{0}\right\rangle}\left(\mathbb{1}_{d-1}-\mathrm{pr}_{z_{0}}\right) \Sigma^{-1} \varphi_{x} \cdot z_{0} .
$$

Thus the coset space $\mathbb{R}_{x, v}^{d-2}:=\mathbb{R}^{d-1} / \mathbb{R}_{z_{0}}$ may be parameterized by the coordinates $v \in \mathbb{R}^{d-2}$ over any $x \in \mathbb{R} \backslash \mathbb{F}_{*}$, and we explicitly retain the fibre index in the notation for convenience.

We now need to unravel the crossed product on the right-hand side of (4.12). From (4.4) and (4.14) it follows that the action of elements $\left(\gamma_{v}, \gamma_{z_{0}}\right) \in \mathbb{Z}^{d-1} \simeq \mathbb{Z}_{v}^{d-2} \oplus \mathbb{Z}_{z_{0}}$ on the coset is given by

$$
\begin{aligned}
& \left(\gamma_{v}, 0,0\right) \cdot(v, x)=\left(v+\gamma_{v}, x\right) \\
& \left(0, \gamma_{z_{0}}, 0\right) \cdot(v, x)=\left(v-\frac{\gamma_{z_{0}}}{\left\langle z_{0}, \Sigma^{-1} \varphi_{x} \cdot z_{0}\right\rangle}\left(\mathbb{1}_{d-1}-\mathrm{pr}_{z_{0}}\right) \Sigma^{-1} \varphi_{x} \cdot z_{0}, x\right) .
\end{aligned}
$$

Applying Theorem 2.6 as in the proof of Lemma 4.16 gives

$$
C\left(\mathbb{R}^{d-1} / \mathbb{Z}^{d-1}\right) \rtimes_{\mathrm{rt} x} \mathbb{R}_{z_{0}} \sim_{\mathrm{M}}\left(C_{0}\left(\mathbb{R}_{x, v}^{d-2}\right) \rtimes_{1 \mathrm{t} x} \mathbb{Z}_{v}^{d-2}\right) \rtimes_{\beta^{x}} \mathbb{Z}_{z_{0}},
$$

where the action of $\mathbb{Z}_{v}^{d-2}$ on the coset $\mathbb{R}_{x, v}^{d-2}$ is given by (4.15), while the action of $\mathbb{Z}_{z_{0}}$ on the crossed product $C_{0}\left(\mathbb{R}_{x, v}^{d-2}\right) \rtimes_{\mathrm{lt}^{x}} \mathbb{Z}_{v}^{d-2}$ is induced by the action on $\mathbb{R}_{x, v}^{d-2}$ given in (4.16) and the trivial action on $\mathbb{Z}_{v}^{d-2}$.

Next, Example 2.14 yields

$$
C_{0}\left(\mathbb{R}_{x, v}^{d-2}\right) \rtimes_{\mathrm{lt}^{x}} \mathbb{Z}_{v}^{d-2} \sim_{\mathrm{M}} C\left(\mathbb{T}^{d-2}\right) .
$$

Since the homomorphism $\sigma_{\mathbb{Z}_{z_{0}}}: \mathbb{Z}_{z_{0}} \rightarrow \mathbb{R}^{+}$is trivial, it is not difficult to see that the Morita equivalence bimodule $\mathcal{M}$ implementing this equivalence is $\mathbb{Z}_{z_{0}}$-equivariant (in the sense of Theo-

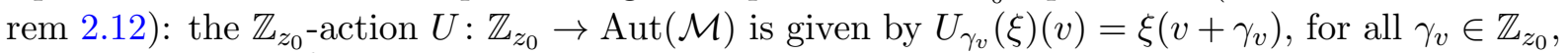
$\xi \in \mathcal{M}$ and $v \in \mathbb{R}_{x, v}^{d-2}$. Applying Theorem 2.12 we conclude that the Morita equivalence (4.17) induces the Morita equivalence

$$
\left(C_{0}\left(\mathbb{R}_{x, v}^{d-2}\right) \rtimes_{\mathrm{lt}^{x}} \mathbb{Z}_{v}^{d-2}\right) \rtimes_{\beta^{x}} \mathbb{Z}_{z_{0}} \sim_{\mathrm{M}} C\left(\mathbb{T}^{d-2}\right) \rtimes_{\mathrm{lt}^{x}} \mathbb{Z}_{z_{0}}
$$

where the action of the group $\mathbb{Z}_{z_{0}}$ on $C\left(\mathbb{T}^{d-2}\right)$ is the pullback of the action on $\mathbb{T}^{d-2}=\mathbb{R}_{x, v}^{d-2} / \mathbb{Z}_{v}^{d-2}$ induced from (4.16).

After rescaling $x$ by $x_{0}$ to give it unit period, this shows that the corresponding algebra of functions on the fiber at $\mathrm{e}^{2 \pi \mathrm{i} x}$ is that of a noncommutative $d$-1-torus $\mathbb{T}_{\vec{\theta}_{z_{0}}(x)}^{d-1}$ with noncommutativity parameter $\vec{\theta}_{z_{0}}(x)$ given by (4.13), see Example 2.2. Thus the $C^{*}$-algebraic T-dual $C\left(\mathbb{T}_{\Lambda_{\mathrm{G}}}\right) \rtimes_{\mathrm{rt}} \mathbb{R}_{z_{0}}$ is a $C^{*}$-algebra bundle of noncommutative tori $\mathrm{A}_{\vec{\theta}_{z_{0}}(x)}=\mathbb{T}_{\vec{\theta}_{z_{0}}(x)}^{d-1}$ over the circle $\mathbb{T}=\left\{\mathrm{e}^{2 \pi \mathrm{i} x} \mid x \in \mathbb{R} / \mathbb{Z}\right\}$. 
What becomes of the non-trivial monodromy (4.4) of the $\mathbb{T}^{d-1}$ fibers parameterized by $z$ in the original Mostow bundle? To answer this question, we write the monodromy matrix $\mathrm{M}=\left(m_{i j}\right) \in \mathrm{SL}(d-1, \mathbb{Z})$ in the block form

$$
\mathrm{M}=\left(\begin{array}{cc}
\mathrm{M}_{\mid d-2} & \vec{m} \\
\vec{m}^{\prime} & m_{d-1 d-1}
\end{array}\right),
$$

where $\mathrm{M}_{\mid d-2}=\left(m_{i j}\right)_{1 \leq i, j \leq d-2}$, while $\vec{m}=\left(m_{i d-1}\right)_{i=1, \ldots, d-2}$ and $\vec{m}^{\prime}=\left(m_{d-1 i}\right)_{i=1, \ldots, d-2}$ are respectively column and row vectors in $\mathbb{Z}^{d-2}$. Below we denote the usual Euclidean inner product on $\mathbb{R}^{d-2}$ by $\langle\cdot, \cdot\rangle$.

Proposition 4.18. The noncommutativity parameter $\vec{\theta}_{\vec{e}_{d-1}}(x)$ from (4.13) varies with a change of coset representative $x \in \mathbb{R} / \mathbb{Z}$ according to its $\mathrm{SL}(d-1, \mathbb{Z})$ orbit under the action of the monodromy matrix (4.19) by (d-2)-dimensional linear fractional transformations

$$
\vec{\theta}_{\vec{e}_{d-1}}(x+1)=\mathrm{M}\left[\vec{\theta}_{\vec{e}_{d-1}}(x)\right]:=\frac{\mathrm{M}_{\mid d-2} \cdot \vec{\theta}_{\vec{e}_{d-1}}(x)+\vec{m}}{\left\langle\vec{m}^{\prime}, \vec{\theta}_{\vec{e}_{d-1}}(x)\right\rangle+m_{d-1 d-1}} .
$$

Under a change of basis of $\mathbb{Z}^{d-1}$ given by a matrix $B \in \mathrm{GL}(d-1, \mathbb{Z})$, the corresponding noncommutativity parameter varies according to

$$
\vec{\theta}_{B \cdot \vec{e}_{d-1}}(x+1)=\left(B^{\mathrm{t}} \mathrm{M} B\right)\left[\vec{\theta}_{B \cdot \vec{e}_{d-1}}(x)\right] .
$$

Proof. The key feature stems from the definition (4.2) of the monodromy matrix M:

$$
\Sigma^{-1} \varphi_{x+x_{0}}=\Sigma^{-1} \varphi_{x_{0}} \varphi_{x}=\mathrm{M} \Sigma^{-1} \varphi_{x}
$$

The statements then follow from straightforward calculations in components.

Remark 4.19. The right-hand side of (4.20) is an example of a linear fractional transformation in higher dimensions, known from complex analysis, see, e.g., [12]. We can show that it defines a Morita equivalence of noncommutative $d$-1-tori from Example 2.11. For this, we introduce the skew-symmetric matrix $\Theta$ corresponding to the vector $\vec{\theta} \in \mathbb{R}^{d-2}$ as in Example 2.2:

$$
\Theta=\left(\begin{array}{cc}
\mathbf{0}_{d-2} & \vec{\theta} \\
-\vec{\theta}^{\mathrm{t}} & 0
\end{array}\right)
$$

We denote by $\boldsymbol{g}_{\mathrm{M}}$ the element of $\mathrm{SO}(d-1, d-1 ; \mathbb{Z})$ corresponding to the monodromy matrix $\mathrm{M} \in \mathrm{SL}(d-1, \mathbb{Z})$ :

$$
\boldsymbol{g}_{\mathrm{M}}=\left(\begin{array}{cc}
\mathrm{M} & \mathbf{0}_{d-1} \\
\mathbf{0}_{d-1} & \left(\mathrm{M}^{\mathrm{t}}\right)^{-1}
\end{array}\right) \in \mathrm{SO}(d-1, d-1 ; \mathbb{Z}) .
$$

Introduce matrices $\boldsymbol{T}_{d-1}$ of order 2 with determinant -1 by

$$
\boldsymbol{T}_{d-1}=\left(\begin{array}{cc}
\mathbb{1}_{d-1}-\mathrm{E}_{d-1} & \mathrm{E}_{d-1} \\
\mathrm{E}_{d-1} & \mathbb{1}_{d-1}-\mathrm{E}_{d-1}
\end{array}\right) \in \mathrm{O}(d-1, d-1 ; \mathbb{Z}),
$$

where $\mathrm{E}_{d-1}$ is the matrix unit whose only non-zero element is $\left(\mathrm{E}_{d-1}\right)_{d-1 d-1}=1$. Finally, define the element $\boldsymbol{M} \in \mathrm{SO}(d-1, d-1 ; \mathbb{Z})$ by

$$
\boldsymbol{M}=\boldsymbol{T}_{d-1} \boldsymbol{g}_{\mathrm{M}} \boldsymbol{T}_{d-1}
$$


Using the adjugate formula for matrix inverses, a straightforward if tedious calculation then shows that the corresponding $\mathrm{SO}(d-1, d-1 ; \mathbb{Z})$ orbit of $\Theta$ reproduces the $(d-2)$-dimensional linear fractional transformation of (4.20):

$$
\boldsymbol{M}[\Theta]=\left(\begin{array}{cc}
\mathbf{0}_{d-2} & \mathrm{M}[\vec{\theta}] \\
-\mathrm{M}[\vec{\theta}]^{\mathrm{t}} & 0
\end{array}\right) \quad \text { with } \quad \mathrm{M}[\vec{\theta}]=\frac{\mathrm{M}_{\mid d-2} \cdot \vec{\theta}+\vec{m}}{\left\langle\vec{m}^{\prime}, \vec{\theta}\right\rangle+m_{d-1 d-1}} .
$$

It follows that, while the fibre noncommutative tori of Theorem 4.17 are not generally identical under a change of representative $x \in \mathbb{R} / \mathbb{Z}$, by Example 2.11 they are always Morita equivalent:

$$
\mathbb{T}_{\vec{\theta}_{\vec{e}_{d-1}}^{d-1}(x+1)} \sim_{\mathrm{M}} \mathbb{T}_{\vec{\theta}_{\vec{e}_{d-1}(x)}^{d-1}}
$$

Recalling that our formulation of topological T-duality takes place in the additive category $\mathscr{K} \mathscr{K}$ from Section 3.2, this has a natural interpretation: The non-trivial monodromy in the automorphism group of the $\mathbb{T}^{d-1}$ fibres of the twisted torus $\mathbb{T}_{\Lambda_{\mathrm{G}}}$ is manifested as a (generally non-trivial) isomorphism in the Morita automorphism group of the fibre noncommutative tori in $\mathscr{K} \mathscr{K}$.

\section{Three-dimensional solvmanifolds and their T-duals}

The goal of this final section is to give some explicit examples in low dimensions of the general formalism we have developed in Section 4, recovering some previously known results in the literature from a new perspective, as well as providing several new examples. Note that tori $\mathbb{T}^{d}$ in any dimension $d$ are covered by our general framework of Section 4.4 for $\mathbb{R}_{y}$-actions: when $\varphi_{x}=\mathbb{1}_{d-1}$ for all $x \in \mathbb{R}$, the torus bundles $\mathbb{T}^{d} \rightarrow \mathbb{T}^{d-n}$ of Proposition 4.13 are trivial. Theorem 4.14 then shows that any $C^{*}$-algebraic T-dual $C\left(\mathbb{T}^{d}\right) \rtimes_{\mathrm{rt}} \mathbb{R}_{y_{0}}$ is isomorphic to $\mathrm{CT}\left(\mathbb{T}^{d-1} \times \mathbb{T}_{y_{0}}, 0\right) \simeq$ $C\left(\mathbb{T}^{d-1} \times \mathbb{T}_{y_{0}}\right) \otimes \mathcal{K}$. Below we apply our formalism to the well-known Mostow fibrations of three-dimensional solvmanifolds.

\subsection{Mostow bundles and $\mathrm{SL}(2, \mathbb{R})$ conjugacy classes}

In three dimensions, solvmanifolds are completely classified, see, e.g., [2]. In particular, by [2, Proposition 5.1] all solvmanifolds in this dimension which are based on connected and simplyconnected Lie groups $\mathrm{G}$ are almost abelian. If $\mathrm{G}=\mathbb{R}^{2} \rtimes_{\varphi} \mathbb{R}$ is abelian, i.e., $\varphi_{x}=\mathbb{1}_{2}$ for all $x \in \mathbb{R}$, then $\mathbb{T}_{\Lambda_{G}} \simeq \mathbb{T}^{3}$ is a torus, while the remaining cases correspond to non-trivial one-parameter group actions

$$
\varphi: \mathbb{R} \longrightarrow \mathrm{SL}(2, \mathbb{R}) .
$$

Elements of $\operatorname{SL}(2, \mathbb{R})$ are classified up to conjugacy by trace, so there are three classes, determined by which of the three types of conjugacy classes of $\operatorname{SL}(2, \mathbb{R})$ that the image of the homomorphism $\varphi$ lands in: parabolic $\left(\left|\operatorname{Tr} \varphi_{x}\right|=2\right.$ for all $\left.x \in \mathbb{R}\right)$, elliptic $\left(\left|\operatorname{Tr} \varphi_{x}\right|<2\right.$ for all $x \neq k \pi / 2$ with $k \in \mathbb{Z})$, or hyperbolic $\left(\left|\operatorname{Tr} \varphi_{x}\right|>2\right.$ for all $x \neq 0$ ), see, e.g., [26].

The existence of cocompact discrete subgroups $\Lambda_{\mathrm{G}}$ of $\mathrm{G}$ is highly restrictive on the allowed homomorphisms $\varphi$; a necessary condition is that there exists $x_{0} \in \mathbb{R}^{\times}$such that $\operatorname{Tr} \varphi_{x_{0}} \in \mathbb{Z}$. The monodromy matrices

$$
\mathrm{M}=\Sigma^{-1} \varphi_{x_{0}} \Sigma=\left(\begin{array}{ll}
a & b \\
c & d
\end{array}\right) \in \mathrm{SL}(2, \mathbb{Z})
$$

are integer matrices that live in the corresponding conjugacy classes of $\operatorname{SL}(2, \mathbb{Z})$. As automorphisms of the torus fibres of the Mostow bundle, in the mapping class group $\operatorname{SL}(2, \mathbb{Z})$, they act on $\mathbb{T}^{2}$ in the following way. Let $\left(\lambda, \lambda^{-1}\right)$ be the eigenvalues of $\mathrm{M}$, which are the roots of the characteristic polynomial $t^{2}-(\operatorname{Tr} \mathrm{M}) t+1$. Then there are three possibilities: 
- Parabolic: In this case $\operatorname{Tr} M= \pm 2$ and $\lambda=\lambda^{-1}= \pm 1$. Then $M$ has an integral eigenvector corresponding to a closed curve on $\mathbb{T}^{2}$ which is invariant under the associated automorphism, and homeomorphisms of this type are Dehn twists of $\mathbb{T}^{2}$.

- Elliptic: In this case $\operatorname{Tr} M=0, \pm 1$, and the eigenvalues $\left(\lambda, \lambda^{-1}\right)$ are complex of modulus 1 . Then the associated automorphism has finite order equal to 2, 3, 4 or 6 , and corresponds to a periodic homeomorphism of $\mathbb{T}^{2}$.

- Hyperbolic: In this case $|\lambda|>1>\left|\lambda^{-1}\right|$. The associated automorphism has no invariant closed curve on $\mathbb{T}^{2}$; it 'stretches' the eigenspace corresponding to $\lambda$ and 'contracts' the eigenspace corresponding to $\lambda^{-1}$. These are called Anasov homeomorphisms of $\mathbb{T}^{2}$.

In this section we shall apply Theorem 4.17 with $z_{0}=\vec{e}_{2}=(0,1)$, which for convenience we combine with Proposition 4.18 and Remark 4.19 to reformulate it as

Theorem 5.1. The $C^{*}$-algebraic T-dual of a three-dimensional solvmanifold $\mathbb{T}_{\Lambda_{\mathrm{G}}}$ with respect to an $\mathbb{R}_{z}$-action with $z_{0}=(0,1)$ is Morita equivalent to a $C^{*}$-algebra bundle of noncommutative two-tori over the circle $\mathbb{T}$ :

$$
C\left(\mathbb{T}_{\Lambda_{\mathrm{G}}}\right) \rtimes_{\mathrm{rt}} \mathbb{R}_{(0,1)} \sim_{M} \coprod_{x \in \mathbb{R} / \mathbb{Z}} \mathbb{T}_{\theta(x)}^{2},
$$

where the noncommutativity parameters $\theta(x) \in \mathbb{R}$ are given by

$$
\theta(x)= \begin{cases}0 & \text { for } x \in \mathbb{F}_{*}, \\ \frac{\left(\Sigma^{-1} \varphi_{x_{0} x}\right)_{12}}{\left(\Sigma^{-1} \varphi_{x_{0} x}\right)_{22}} & \text { for } x \in \mathbb{R} \backslash \mathbb{F}_{*},\end{cases}
$$

where $\left(\Sigma^{-1} \varphi_{x_{0} x}\right)_{i j}$ for $i, j \in\{1,2\}$ denote the matrix elements of $\Sigma^{-1} \varphi_{x_{0} x}$. The noncommutativity parameter $\theta(x)$ varies under a change of coset representative $x \in \mathbb{R} / \mathbb{Z}$ according to its $\mathrm{SL}(2, \mathbb{Z})$ orbit under the corresponding monodromy matrix (5.1):

$$
\theta(x+1)=\mathrm{M}[\theta(x)]=\frac{a \theta(x)+b}{c \theta(x)+d} .
$$

By Example 2.10 the corresponding fibre noncommutative tori are Morita equivalent:

$$
\mathbb{T}_{\theta(x+1)}^{2} \sim_{M} \mathbb{T}_{\theta(x)}^{2},
$$

and so isomorphic in the category $\mathscr{K} \mathscr{K}$.

The $C^{*}$-algebraic T-dual in these instances has more structure in general than the original algebra of functions $C\left(\mathbb{T}_{\Lambda_{\mathrm{G}}}\right)$, as a consequence of

Proposition 5.2. The noncommutative torus bundle $\coprod_{x \in \mathbb{R} / \mathbb{Z}} \mathbb{T}_{\theta(x)}^{2}$ is a $C\left(\mathbb{T}^{2}\right)$-algebra.

Proof. We can also describe these noncommutative torus bundles via (strict) deformation quantization: Performing an identical calculation to that of Example 2.1, we may describe the fibrewise crossed product (4.18) (with $d=3$ ) explicitly in terms of a star-product, and hence the $C^{*}$-algebra bundle of Theorem 5.1 as a deformation of the algebra of functions $C\left(\mathbb{T} \times \mathbb{T}^{2}\right)$ on the trivial torus bundle over $\mathbb{T}$ by regarding the convolution algebra $C_{\mathrm{c}}(\mathbb{T} \times \mathbb{T} \times \mathbb{Z})$ as the space of functions $C\left(\mathbb{T} \times \mathbb{T}^{2}\right)$ equipped with the star-product

$$
\begin{aligned}
&\left(f \star_{\theta} g\right)\left(x, v_{1}, v_{2}\right)= \sum_{\left(p_{1}, p_{2}\right) \in \mathbb{Z}^{2}} \mathrm{e}^{2 \pi \mathrm{i}\left(p_{1} v_{1}+p_{2} v_{2}\right)} \\
& \times \sum_{\left(q_{1}, q_{2}\right) \in \mathbb{Z}^{2}} f_{q_{1}, q_{2}}(x) g_{p_{1}-q_{1}, p_{2}-q_{2}}(x) \mathrm{e}^{2 \pi \mathrm{i} \theta(x)\left(p_{1}-q_{1}\right) q_{2}},
\end{aligned}
$$


where we used the fibrewise Fourier transformation

$$
f\left(x, v_{1}, v_{2}\right)=\sum_{\left(p_{1}, p_{2}\right) \in \mathbb{Z}^{2}} f_{p_{1}, p_{2}}(x) \mathrm{e}^{2 \pi \mathrm{i}\left(p_{1} v_{1}+p_{2} v_{2}\right)},
$$

with functions $f_{p_{1}, p_{2}}: \mathbb{T} \rightarrow \mathbb{C}$. In this formulation of the $C^{*}$-algebraic T-dual, it is immediately evident that there is an injection $C\left(\mathbb{T}^{2}\right) \hookrightarrow \bigsqcup_{x \in \mathbb{R} / \mathbb{Z}} \mathbb{T}_{\theta(x)}^{2}$ as the space of functions which are independent of the coordinate $v_{1}$ (or $v_{2}$ ); for such functions, the sums in (5.3) truncate to $p_{1}=q_{1}=0$ (or $p_{2}=q_{2}=0$ ) and the noncommutative star-product reduces to the commutative pointwise product of functions in $C\left(\mathbb{T}^{2}\right)$. This defines $C^{*}$-algebra monomorphisms making $\coprod_{x \in \mathbb{R} / \mathbb{Z}} \mathbb{T}_{\theta(x)}^{2}$ into a $C\left(\mathbb{T}^{2}\right)$-algebra.

The remainder of this paper is devoted to providing illustrations of Theorem 5.1, through explicit calculations in each of the three conjugacy classes of $\operatorname{SL}(2, \mathbb{R})$. The features differ for each conjugacy class so we consider them individually in turn.

\subsection{Parabolic torus bundles}

We start with the best studied example in the literature, which is based on the nilpotent Heisenberg group. In string theory it is T-dual to the three-torus $\mathbb{T}^{3}$ with $H$-flux by the standard Buscher rules (see, e.g., [27]), and in topological T-duality it gives the basic example of a noncommutative principal torus bundle [15, 21,32]. Here we shall give a new algebraic perspective on both these T-duals by applying our formalism of topological T-duality directly to the Heisenberg nilmanifold. This class has several features that make it special among the three-dimensional solvmanifolds, which we explain in detail.

\section{Heisenberg nilmanifolds}

The three-dimensional Heisenberg group Heis(3) is the nilpotent Lie group whose Mostow bundle structure is based on the semi-direct product

$$
\operatorname{Heis}(3)=\mathbb{R}^{2} \rtimes_{\varphi} \mathbb{R} \quad \text { with } \quad \varphi_{x}=\left(\begin{array}{ll}
1 & x \\
0 & 1
\end{array}\right) .
$$

Since $\left|\operatorname{Tr} \varphi_{x}\right|=2$, the matrix $\varphi_{x}$ parameterizes a parabolic conjugacy class of $\operatorname{SL}(2, \mathbb{R})$. Here we denote the coordinates on the group manifold of $N=\mathbb{R}^{2}$ by $(y, z)$. The group multiplication on Heis(3) is then given by

$$
(x, y, z)\left(x^{\prime}, y^{\prime}, z^{\prime}\right)=\left(x+x^{\prime}, y+y^{\prime}+x z^{\prime}, z+z^{\prime}\right),
$$

and the inverse of a group element is

$$
(x, y, z)^{-1}=(-x, x z-y,-z) .
$$

In this case it is clear that for

$$
x_{0}=m
$$

with $m \in \mathbb{Z}^{\times}$, the matrix $\varphi_{x_{0}}$ is integer-valued and hence may be taken as monodromy matrix $\mathrm{M}_{m}$ of infinite order with $\Sigma=\mathbb{1}_{2}$ :

$$
\mathrm{M}_{m}=\varphi_{m}=\left(\begin{array}{cc}
1 & m \\
0 & 1
\end{array}\right)
$$


The Heisenberg nilmanifold $\mathbb{T}_{\text {Heis }_{m}(3 ; \mathbb{Z})}$ is the compact space obtained as the quotient of Heis(3) with respect to the lattice given by the discrete Heisenberg group

$$
\operatorname{Heis}_{m}(3 ; \mathbb{Z}):=\{(m \alpha, \beta, \gamma) \in \operatorname{Heis}(3) \mid \alpha, \beta, \gamma \in \mathbb{Z}\} .
$$

The equivalence relation is given by the left action of $\operatorname{Heis}_{m}(3 ; \mathbb{Z})$, which leads to the local coordinate identifications under the action of the generators of $\operatorname{Heis}_{m}(3 ; \mathbb{Z})$ given by

$$
\begin{aligned}
& (x, y, z) \longmapsto(x+m, y+m z, z), \\
& (x, y, z) \longmapsto(x, y+1, z), \\
& (x, y, z) \longmapsto(x, y, z+1) .
\end{aligned}
$$

Geometrically, this exhibits the Heisenberg nilmanifold as a non-trivial principal $\mathbb{T}^{2}$-bundle $\mathbb{T}_{\text {Heis }_{m}(3 ; \mathbb{Z})} \rightarrow \mathbb{T}$. Using the algorithm described in Section 4.4 , the relation matrix

$$
\mathrm{A}_{m}=\mathrm{M}_{m}-\mathbb{1}_{2}
$$

has rank $r=1$ with elementary divisors $m_{1}=m$ and $m_{2}=0$, and the first homology group of $\mathbb{T}_{\text {Heis }_{m}(3 ; \mathbb{Z})}$ can thus be presented as the $\mathbb{Z}$-module

$$
\mathrm{H}_{1}\left(\mathbb{T}_{\text {Heis }_{m}(3 ; \mathbb{Z})}, \mathbb{Z}\right) \simeq \mathbb{Z} \oplus\left(\mathbb{Z} \oplus \mathbb{Z}_{m}\right),
$$

with respective free generators denoted $e_{x}$ and $e_{y}$, and torsion generator $e_{z}$ of order $m, m e_{z}=0$.

Dually, the topology of the nilmanifold is captured by its algebra of functions, which can be computed as the subalgebra of invariant functions on the Heisenberg group Heis(3) with respect to the left action of the lattice $\operatorname{Heis}_{m}(3 ; \mathbb{Z})$ :

$$
C\left(\mathbb{T}_{\text {Heis }_{m}(3 ; \mathbb{Z})}\right)=C_{0}(\operatorname{Heis}(3))^{\operatorname{Heis}_{m}(3 ; \mathbb{Z})} .
$$

Harmonic analysis on the nilmanifold can be used to determine the Fourier decomposition of any function $f \in C\left(\mathbb{T}_{\text {Heis }_{m}(3 ; \mathbb{Z})}\right)$. Invariance under the generators $(5.5)$ of $\operatorname{Heis}_{m}(3 ; \mathbb{Z})$ forces the expansion to take the form

$$
\mathbf{f}(x, y, z)=\sum_{k \in \mathbb{Z}_{m}} \sum_{p, q \in \mathbb{Z}} \mathrm{f}_{p, k}(x+m q) \mathrm{e}^{2 \pi \mathrm{i} p y+2 \pi \mathrm{i}(k+m p q) z},
$$

for functions $\mathrm{f}_{p, k}: \mathbb{R} \rightarrow \mathbb{C}$ vanishing at infinity. From this expression we can easily see the $C(\mathbb{T})$ algebra structure of $C\left(\mathbb{T}_{\text {Heis }_{m}(3 ; \mathbb{Z})}\right)$ : Restricting (5.6) to $y=z=0$ determines a function on the circle $\mathbb{T}=\left\{\left(\mathrm{e}^{2 \pi \mathrm{i} x / m}, 1,1\right) \in \mathbb{T}_{\text {Heis }_{m}(3 ; \mathbb{Z})}\right\}$ and defines a $C^{*}$-algebra monomorphism $C(\mathbb{T}) \hookrightarrow$ $C\left(\mathbb{T}_{\text {Heis }_{m}(3 ; \mathbb{Z})}\right)$. On the other hand, the evaluation of $(5.6)$ at $z=0$ yields a function on the two-torus $\mathbb{T}^{2}=\left\{\left(\mathrm{e}^{2 \pi \mathrm{i} x / m}, \mathrm{e}^{2 \pi \mathrm{i} y}, 1\right) \in \mathbb{T}_{\text {Heis }_{m}(3 ; \mathbb{Z})}\right\}$ and defines a $C^{*}$-algebra monomorphism $C\left(\mathbb{T}^{2}\right) \hookrightarrow C\left(\mathbb{T}_{\text {Heis }_{m}(3 ; \mathbb{Z})}\right)$ making $C\left(\mathbb{T}_{\text {Heis }_{m}(3 ; \mathbb{Z})}\right)$ into a $C\left(\mathbb{T}^{2}\right)$-algebra in this case.

Following the prescriptions of Sections 4.5 and 4.6, we shall now study the known T-duals of the Heisenberg nilmanifold through the formalism of topological T-duality discussed in Section 3.

\section{$\mathbb{R}_{y}$-action: $\mathbb{T}^{3}$ with $H$-flux}

The one-parameter subgroup

$$
\operatorname{ker}\left(\mathrm{A}_{m}\right)=\mathbb{R}_{(1,0)}:=\{(0, \lambda, 0) \in \operatorname{Heis}(3)\}
$$

is the center of Heis $(3)$. The quotient group Heis $(3) / \mathbb{R}_{(1,0)}$ can be parameterized by equivalence classes $[(x, 0, z)]$, and the multiplication law

$$
[(x, 0, z)]\left[\left(x^{\prime}, 0, z^{\prime}\right)\right]=\left[\left(x+x^{\prime}, 0, z+z^{\prime}\right)\right]
$$


is that of the abelian Lie group Heis $(3) / \mathbb{R}_{(1,0)} \simeq \mathbb{R}^{2}$. It follows that the quotient $\mathbb{T}_{\text {Heis }_{m}(3 ; \mathbb{Z})} / \mathbb{R}_{(1,0)}$ is the two-dimensional torus $\mathbb{T}^{2}$ with coordinates $\left(\mathrm{e}^{2 \pi \mathrm{i} x / m}, \mathrm{e}^{2 \pi \mathrm{i} z}\right)$. The corresponding principal circle bundle $p_{(1,0)}: \mathbb{T}_{\text {Heis }_{m}(3 ; \mathbb{Z})} \rightarrow \mathbb{T}^{2}$, with fibre coordinate $\mathrm{e}^{2 \pi \mathrm{i} y}$, is the standard realization of the Heisenberg nilmanifold as a $\mathbb{T}$-fibration over $\mathbb{T}^{2}$ of degree $m$ : the connection

$$
\kappa_{(1,0)}=-\mathrm{d} y+x \mathrm{~d} z
$$

of Theorem 4.14 has curvature

$$
\mathrm{d} \kappa_{(1,0)}=\mathrm{d} x \wedge \mathrm{d} z
$$

and so the first Chern class $c_{1}\left(p_{(1,0)}\right)$ is $m$ times the standard generator of $\mathrm{H}^{2}\left(\mathbb{T}^{2}, \mathbb{Z}\right) \simeq \mathbb{Z}$. By Theorem 4.14 it follows that the $C^{*}$-algebraic T-dual

$$
C\left(\mathbb{T}_{\text {Heis }_{m}(3 ; \mathbb{Z})}\right) \rtimes_{\mathrm{rt}} \mathbb{R}_{(1,0)} \simeq \mathrm{CT}\left(\mathbb{T}^{3}, m\right)
$$

is a continuous-trace algebra with spectrum $\mathbb{T}^{3}$ and Dixmier-Douady class equal to $m$ times the standard generator of $\mathrm{H}^{3}\left(\mathbb{T}^{3}, \mathbb{Z}\right) \simeq \mathbb{Z}$. Thus in this case we recover the standard T-duality between the three-torus $\mathbb{T}^{3}$ with $H$-flux and the Heisenberg nilmanifold viewed as a circle bundle. The correspondence space construction is given by Proposition 4.15 with $\mathbb{T}_{\Lambda_{G(1,0)}}=\mathbb{T}^{2}$.

\section{$\mathbb{R}_{z^{-} \text {-action: Noncommutative principal torus bundles }}$}

We shall now recover the known noncommutative principal torus bundle which is T-dual to $\mathbb{T}_{\text {Heis }_{m}(3 ; \mathbb{Z})}$ from a new perspective, by directly working with the algebraic description of the nilmanifold. For this, we follow our prescription for $\mathbb{R}_{z}$-actions from Section 4.6 and consider the one-parameter subgroup $\mathbb{R}_{(0,1)}$ of Heis $(3)$ given by

$$
\mathbb{R}_{(0,1)}=\{(0,0, \zeta) \in \operatorname{Heis}(3)\},
$$

whose right action by multiplication generates

$$
(x, y, z)(0,0, \zeta)=(x, y+x \zeta, z+\zeta) .
$$

The quotient of this action to $\mathbb{T}_{\text {Heis }_{m}(3 ; \mathbb{Z})}$ fixes every point of the form $\left(\mathrm{e}^{2 \pi \mathrm{i} n / m}, \mathrm{e}^{2 \pi \mathrm{i} y}, \mathrm{e}^{2 \pi \mathrm{i} z}\right) \in$ $\mathbb{T}^{2} \subset \mathbb{T}_{\text {Heis }_{m}(3 ; \mathbb{Z})}$ for $n \in \mathbb{Z}$ with isotropy subgroup

$$
\mathbb{Z}_{(0,1)}=\mathbb{R}_{(0,1)} \cap \operatorname{Heis}_{m}(3 ; \mathbb{Z}) .
$$

In this case $\mathbb{F}_{*}=\varnothing$, and Theorem 5.1 thus identifies the $C^{*}$-algebraic T-dual through the Morita equivalence

$$
C\left(\mathbb{T}_{\text {Heis }_{m}(3 ; \mathbb{Z})}\right) \rtimes_{\mathrm{rt}} \mathbb{R}_{(0,1)} \sim_{\mathrm{M}} \coprod_{x \in \mathbb{R} / \mathbb{Z}} \mathbb{T}_{m x}^{2}
$$

of $C(\mathbb{T})$-algebras.

We can explicitly check the anticipated monodromy (5.2): Under a change of coset representative $x \in \mathbb{R} / \mathbb{Z}$ of the fibres of the noncommutative torus bundle $\coprod_{x \in \mathbb{R} / \mathbb{Z}} \mathbb{T}_{\theta_{m}(x)}^{2}$, the noncommutativity parameter changes

$$
\theta_{m}(x+1)=\theta_{m}(x)+m=\mathrm{M}_{m}\left[\theta_{m}(x)\right]
$$

by the $\operatorname{SL}(2, \mathbb{Z})$ orbit of $\theta_{m}(x)=m x$ under the monodromy matrix $\mathrm{M}_{m}$ given by (5.4), and by Example 2.10 the fiber noncommutative tori are identical: $\mathbb{T}_{\theta_{m}(x+1)}^{2}=\mathbb{T}_{\theta_{m}(x)}^{2}$. Hence in this 
case the non-trivial monodromy in the automorphism group of the $\mathbb{T}^{2}$ fibres of the twisted torus is implemented trivially as an equality in its T-dual $C^{*}$-algebra bundle.

It is well known that these $C^{*}$-bundles are isomorphic to the convolution $C^{*}$-algebras of the corresponding integer Heisenberg groups, and indeed this is precisely the original description of these T-dual noncommutative torus bundles from [32]. For later comparison, it is instructive to explicitly demonstrate this result using our scheme for topological T-duality, which results in

Proposition 5.3. The $C^{*}$-algebraic T-dual $C\left(\mathbb{T}_{\text {Heis }_{m}(3 ; \mathbb{Z})}\right) \rtimes_{\mathrm{rt}} \mathbb{R}_{(0,1)}$ is Morita equivalent to the group $C^{*}$-algebra $C^{*}\left(\operatorname{Heis}_{m}(3 ; \mathbb{Z})\right)$.

Proof. The crux of the proof hinges on the fact that the parabolic torus bundles are the only class in three dimensions for which a fibrewise analysis is not necessary and the Morita isomorphisms can be implemented directly in the category $\mathscr{K} \mathscr{K}$. For this, we observe that any element $(x, y, z) \in$ Heis $(3)$ can be uniquely factorized as

$$
(x, y, z)=(x, y-x z, 0)(0,0, z)=:(x, v, 0)(0,0, z),
$$

with the action of the subgroup $\mathbb{R}_{(0,1)}$ on the normal abelian subgroup $\mathbb{R}_{(x, v)}^{2}:=\{(x, v, 0) \in$ Heis(3)\} given by

$$
\operatorname{Ad}_{z}(x, v, 0):={ }^{(0,0, z)}(x, v, 0)=(0,0, z)(x, v, 0)(0,0,-z)=(x, v-x z, 0) .
$$

It follows that the Heisenberg group can alternatively be presented as the semi-direct product Heis $(3)=\mathbb{R}_{(x, v)}^{2} \mathbb{R}_{(0,1)}$. Correspondingly, its lattice is also a semi-direct product $\operatorname{Heis}_{m}(3 ; \mathbb{Z})=$ $\mathbb{Z}_{(x, v)}^{2} \rtimes_{\text {Ad }} \mathbb{Z}_{(0,1)}$, where $\mathbb{Z}_{(x, v)}^{2}=\mathbb{R}_{(x, v)}^{2} \cap \operatorname{Heis}_{m}(3 ; \mathbb{Z})$. Thus the Morita equivalence from (3.3) can be expressed as

$$
\begin{aligned}
C\left(\mathbb{T}_{\text {Heis }_{m}(3 ; \mathbb{Z})}\right) \rtimes_{\mathrm{rt}} \mathbb{R}_{(0,1)} & \sim_{\mathrm{M}} C_{0}\left(\operatorname{Heis}(3) / \mathbb{R}_{(0,1)}\right) \rtimes_{\mathrm{lt}} \operatorname{Heis}_{m}(3 ; \mathbb{Z}) \\
& \sim{ }_{\mathrm{M}} C_{0}\left(\mathbb{R}_{(x, v)}^{2}\right) \rtimes_{\mathrm{lt}}\left(\mathbb{Z}_{(x, v)}^{2} \rtimes_{\mathrm{Ad}} \mathbb{Z}_{(0,1)}\right) \\
& \simeq\left(C_{0}\left(\mathbb{R}_{(x, v)}^{2}\right) \rtimes_{\mathrm{lt}} \mathbb{Z}_{(x, v)}^{2}\right) \rtimes_{\beta} \mathbb{Z}_{(0,1)},
\end{aligned}
$$

where in the last line we have further applied Theorem 2.6. Since the groups $\mathbb{Z}_{(x, v)}^{2}$ and $\mathbb{Z}_{(0,1)}$ are discrete, the homomorphism $\sigma_{\mathbb{Z}_{(0,1)}}$ from (2.5) is trivial, and so the $\mathbb{Z}_{(0,1)}$-action on $C_{0}\left(\mathbb{R}_{(x, v)}^{2}\right) \rtimes_{\text {lt }}$ $\mathbb{Z}_{(x, v)}^{2}$ is the canonical action obtained from the diagonal action of $\mathbb{Z}_{(0,1)}$ on $\mathbb{Z}_{(x, v)}^{2} \times \mathbb{R}_{(x, v)}^{2}$ :

$$
\beta_{\zeta}(f)((m \alpha, \nu),(x, v))=f((m \alpha, \nu+m \alpha \zeta),(x, v+x \zeta))
$$

for all $\zeta \in \mathbb{Z}_{(0,1)},(m \alpha, \nu) \in \mathbb{Z}_{(x, v)}^{2},(x, v) \in \mathbb{R}_{(x, v)}^{2}$ and $f \in C_{\mathrm{c}}\left(\mathbb{Z}_{(x, v)}^{2} \times \mathbb{R}_{(x, v)}^{2}\right) \subset C_{0}\left(\mathbb{R}_{(x, v)}^{2}\right) \rtimes_{\mathrm{lt}}$ $\mathbb{Z}_{(x, v)}^{2}$.

By Example 2.14 there is a Morita equivalence

$$
C_{0}\left(\mathbb{R}_{(x, v)}^{2}\right) \rtimes_{\mathrm{lt}} \mathbb{Z}_{(x, v)}^{2} \sim_{\mathrm{M}} C\left(\mathbb{T}^{2}\right) .
$$

Since the homomorphism $\sigma_{\mathbb{Z}_{(0,1)}}: \mathbb{Z}_{(0,1)} \rightarrow \mathbb{R}^{+}$is trivial, it follows that the Morita equivalence bimodule $\mathcal{M}$ implementing this equivalence is $\mathbb{Z}_{(0,1)}$-equivariant: the $\mathbb{Z}_{(0,1)}$-action $U: \mathbb{Z}_{(0,1)} \rightarrow$ $\operatorname{Aut}(\mathcal{M})$ is given by $U_{\zeta}(\xi)(x, v)=\xi(x, v+x \zeta)$, for all $\zeta \in \mathbb{Z}_{(0,1)}, \xi \in \mathcal{M}$ and $(x, v) \in \mathbb{R}_{(x, v)}^{2}$. Applying Theorem 2.12 we conclude that the Morita equivalence (5.9) induces the Morita equivalence $\left(C_{0}\left(\mathbb{R}_{(x, v)}^{2}\right) \rtimes_{l t} \mathbb{Z}_{(x, v)}^{2}\right) \rtimes_{\beta} \mathbb{Z}_{(0,1)} \sim_{\mathrm{M}} C\left(\mathbb{T}^{2}\right) \rtimes_{\mathrm{Ad}^{*}} \mathbb{Z}_{(0,1)}$, where the action of the group $\mathbb{Z}_{(0,1)}$ on $C\left(\mathbb{T}^{2}\right)$ is the pullback of the action on $\mathbb{T}^{2}=\mathbb{R}_{(x, v)}^{2} / \mathbb{Z}_{(x, v)}^{2}$ induced from the left multiplication of $\mathbb{Z}_{(0,1)}$ on Heis $(3)$. 
Substituting into (5.8) we thus obtain

$$
C\left(\mathbb{T}_{\text {Heis }_{m}(3 ; \mathbb{Z})}\right) \rtimes_{\mathrm{rt}} \mathbb{R}_{(0,1)} \sim_{\mathrm{M}} C\left(\mathbb{T}^{2}\right) \rtimes_{\mathrm{Ad}^{*}} \mathbb{Z}_{(0,1)} .
$$

Now we implement Pontryagin duality via Fourier transform and apply (2.8) to the right-hand side of (5.10) to obtain

$$
C\left(\mathbb{T}^{2}\right) \rtimes_{\mathrm{Ad}^{*}} \mathbb{Z}_{(0,1)} \simeq C^{*}\left(\mathbb{Z}_{(x, v)}^{2}\right) \rtimes_{\widehat{\mathrm{Ad}^{*}}} \mathbb{Z}_{(0,1)},
$$

where we used the fact that $\widehat{\sigma}_{\mathbb{Z}_{(0,1)}}: \mathbb{Z}_{(0,1)} \rightarrow \mathbb{R}^{+}$is trivial since $\mathbb{Z}_{(0,1)}$ is discrete. Applying Theorem 2.5, we then arrive at

$$
C\left(\mathbb{T}_{\text {Heis }_{m}(3 ; \mathbb{Z})}\right) \rtimes_{\mathrm{rt}} \mathbb{R}_{(0,1)} \sim_{\mathrm{M}} C^{*}\left(\mathbb{Z}_{(x, v)}^{2} \rtimes_{\mathrm{Ad}} \mathbb{Z}_{(0,1)}\right)=C^{*}\left(\text { Heis }_{m}(3 ; \mathbb{Z})\right),
$$

as required.

Remark 5.4. The star-products (5.3) with $\theta(x)=\theta_{m}(x)=m x$ are equivalent to the starproducts of $[21,28,31]$. In $[15,21]$, it is shown that the description of Proposition 5.3 defines a noncommutative principal $\mathbb{T}^{2}$-bundle (cf. Example 4.9). It is also shown in [15] that the monodromy (5.7), while acting trivially at the purely algebraic level, has a non-trivial action on the K-theory group of the $C^{*}$-algebra bundle $\bigsqcup_{x \in \mathbb{R} / \mathbb{Z}} \mathbb{T}_{m x}^{2}$, viewed as a bundle of abelian groups over $\mathbb{T}$; a physical picture of this action in terms of monodromies of fiber D-branes is discussed in $[28]$.

\section{Noncommutative correspondences}

We come now to the noncommutative correspondences underlying this topological T-duality. For this, we recall that both $C\left(\mathbb{T}_{\text {Heis }_{m}(3 ; \mathbb{Z})}\right)$ and $C\left(\mathbb{T}^{2}\right) \rtimes_{\mathrm{Ad}^{*}} \mathbb{Z}_{(0,1)}$ are $C\left(\mathbb{T}^{2}\right)$-algebras, with $\mathbb{T}^{2}$ parameterized by the local coordinates $(x, y)$. We will show that the noncommutative correspondence is given by the balanced tensor product

$$
C\left(\mathbb{T}_{\text {Heis }_{m}(3 ; \mathbb{Z})}\right) \rtimes_{\mathrm{rt}} \mathbb{Z}_{(0,1)} \simeq C\left(\mathbb{T}_{\text {Heis }_{m}(3 ; \mathbb{Z})}\right) \otimes_{C\left(\mathbb{T}^{2}\right)} \coprod_{x \in \mathbb{R} / \mathbb{Z}} \mathbb{T}_{m x}^{2}
$$

over $C\left(\mathbb{T}^{2}\right)$. Note that here the subgroup $\mathbb{Z}_{(0,1)}$ acts non-trivially on the algebra of functions $C\left(\mathbb{T}_{\text {Heis }_{m}(3 ; \mathbb{Z})}\right)$, in contrast to our previous examples. We consider elements of the convolution algebra $C_{\mathrm{c}}\left(\mathbb{T}_{\text {Heis }_{m}(3 ; \mathbb{Z})} \times \mathbb{Z}_{(0,1)}\right)$ as sequences $f=\left\{f_{\tilde{q}}\right\}_{\tilde{q} \in \mathbb{Z}_{(0,1)}}$ of functions $f_{\tilde{q}}: \mathbb{T}_{\text {Heis }_{m}(3 ; \mathbb{Z})} \rightarrow \mathbb{C}$ with the convolution product

$$
(f \star g)_{\tilde{q}}(x, y, z)=\sum_{\tilde{p} \in \mathbb{Z}} f_{\tilde{p}}(x, y, z) g_{\tilde{q}-\tilde{p}}(x, y-\tilde{p} m x, z) .
$$

We write the Fourier transformation

$$
f(x, y, z, \tilde{z}):=\sum_{\tilde{q} \in \mathbb{Z}} f_{\tilde{q}}(x, y, z) \mathrm{e}^{2 \pi \mathrm{i} \tilde{q} \tilde{z}}
$$

for functions on $\mathbb{T}_{\text {Heis }_{m}(3 ; \mathbb{Z})} \times \widetilde{\mathbb{T}}_{z}$, and define the star-product

$$
(f \star g)(x, y, z, \tilde{z})=\sum_{\tilde{q} \in \mathbb{Z}}(f \star g)_{\tilde{q}}(x, y, z) \mathrm{e}^{2 \pi \mathrm{i} \tilde{q} \tilde{z}} .
$$

We further use the harmonic expansion of functions on the nilmanifold given from (5.6) by

$$
f_{\tilde{q}}(x, y, z)=\sum_{k \in \mathbb{Z}_{m}} \sum_{p, q \in \mathbb{Z}} f_{\tilde{q} ; p, k}(x+q) \mathrm{e}^{2 \pi \mathrm{i} q y+2 \pi \mathrm{i}(k+m p q) z} .
$$


After some elementary manipulations, we can then write the star-product on the space of functions $C\left(\mathbb{T}_{\text {Heis }_{m}(3 ; \mathbb{Z})} \times \widetilde{\mathbb{T}}_{z}\right)$ as

$$
\begin{aligned}
& (f \star g)(x, y, z, \tilde{z})=\sum_{l \in \mathbb{Z}_{m}} \sum_{r, s, \tilde{p} \in \mathbb{Z}} \mathrm{e}^{2 \pi \mathrm{i} r y+2 \pi \mathrm{i}(l+m r s) z} \mathrm{e}^{2 \pi \mathrm{i} \tilde{p} \tilde{z}} \\
& \quad \times \sum_{k \in \mathbb{Z}_{m}} \sum_{p, q, \tilde{q} \in \mathbb{Z}} f_{\tilde{q} ; p, k}(x+q) g_{\tilde{p}-\tilde{q} ; r-p, l-k}(x+q-s) \mathrm{e}^{2 \pi \mathrm{i} m r(q-s) z} \mathrm{e}^{-2 \pi \mathrm{i} m x(r-p) \tilde{q}} .
\end{aligned}
$$

We can define an injection $C\left(\mathbb{T}_{\text {Heis }_{m}(3 ; \mathbb{Z})}\right) \hookrightarrow C\left(\mathbb{T}_{\text {Heis }_{m}(3 ; \mathbb{Z})} \times \widetilde{\mathbb{T}}_{z}\right)$ as the space of functions which are independent of the coordinate $\tilde{z}$. For these functions the sums in (5.11) truncate to $\tilde{p}=\tilde{q}=0$, and one recovers the pointwise product of functions in $C\left(\mathbb{T}_{\text {Heis }_{m}(3 ; \mathbb{Z})}\right)$. On the other hand, we can include the noncommutative algebra $\coprod_{x \in \mathbb{R} / \mathbb{Z}} \mathbb{T}_{m x}^{2} \hookrightarrow C\left(\mathbb{T}_{\text {Heis }_{m}(3 ; \mathbb{Z})} \times \widetilde{\mathbb{T}}_{z}\right)$ as the space of functions which are independent of the coordinate $z$, and one sees that (5.11) truncates for $l=k=0$ and $s=q=0$ to the star-product (5.3) with $\theta(x)=\theta_{m}(x)=m x$. Altogether, the noncommutative correspondences are induced by the diagram

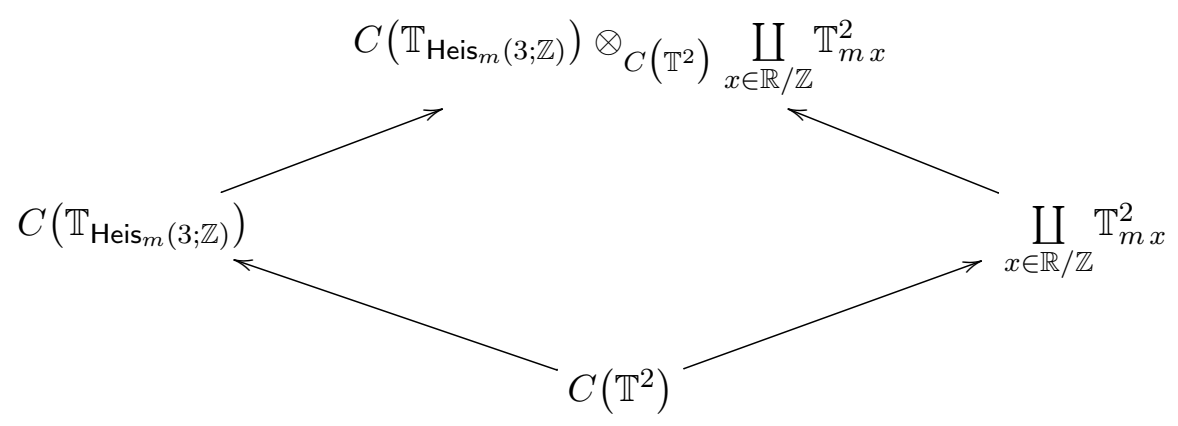

where all arrows are $*$-monomorphisms of $C^{*}$-algebras.

\subsection{Elliptic torus bundles}

We shall now illustrate Theorem 5.1 for the three-dimensional solvmanifolds based on the Euclidean group in two dimensions, which have no classical Hausdorff T-duals and whose noncommutative T-dual fibration was first discussed in [28] for the case of the $\mathbb{Z}_{4}$ elliptic monodromy. Here we shall recover this noncommutative geometry rigorously from our algebraic framework, and moreover extend it to the $\mathbb{Z}_{2}$ and $\mathbb{Z}_{6}$ elliptic monodromies which have not been considered previously.

The Euclidean group ISO(2) in two dimensions is the three-dimensional almost abelian solvable Lie group

$$
\operatorname{ISO}(2)=\mathbb{R}^{2} \rtimes_{\varphi} \mathbb{R} \quad \text { with } \quad \varphi_{x}=\left(\begin{array}{cc}
\cos x & \sin x \\
-\sin x & \cos x
\end{array}\right) .
$$

Since $\left|\operatorname{Tr} \varphi_{x}\right|<2$ for $x \notin \pi \mathbb{Z}$, the rotations $\varphi_{x}$ parameterize an elliptic conjugacy class of $\operatorname{SL}(2, \mathbb{R})$. Here we shall denote coordinates on the group manifold of $\mathbf{N}=\mathbb{R}^{2}$ by $z=\left(z_{1}, z_{2}\right)$. The group multiplication on $\mathrm{ISO}(2)$ is then

$$
\left(x, z_{1}, z_{2}\right)\left(x^{\prime}, z_{1}^{\prime}, z_{2}^{\prime}\right)=\left(x+x^{\prime}, z_{1}+z_{1}^{\prime} \cos x+z_{2}^{\prime} \sin x, z_{2}-z_{1}^{\prime} \sin x+z_{2}^{\prime} \cos x\right)
$$

and the inverse of a group element is

$$
\left(x, z_{1}, z_{2}\right)^{-1}=\left(-x,-z_{1} \cos x+z_{2} \sin x,-z_{1} \sin x-z_{2} \cos x\right) .
$$

The existence of lattices in ISO $(2)$ is highly restrictive $[26,28]$. They require angles $x_{0} \in \mathbb{R}^{\times}$ for which $2 \cos x_{0} \in \mathbb{Z}$, i.e., $\cos x_{0}=0, \pm \frac{1}{2}, \pm 1$. For our purposes, there are three inequivalent 
choices of non-trivial elliptic monodromies, which are characterized by matrices $\mathrm{M} \in \mathrm{SL}(2, \mathbb{Z})$ of finite order in the cyclic subgroups $\mathbb{Z}_{2}, \mathbb{Z}_{4}$ and $\mathbb{Z}_{6}$. They lead to three types of Euclidean solvmanifolds, according to the order of the monodromy in the fibre of the associated Mostow bundle. A crucial distinction from the case of the Heisenberg nilmanifold is that here the Mostow fibrations are not principal $\mathbb{T}^{2}$-bundles. We consider each of the three cases separately in turn.

\section{Euclidean $\mathbb{Z}_{\mathbf{2}}$-solvmanifolds}

We observe that for

$$
x_{0}=m \pi
$$

with $m \in \mathbb{Z}^{\times}$, the matrix $\varphi_{x_{0}}$ is integer-valued, and so may be set equal to $\mathrm{M}$ with $\Sigma=\mathbb{1}_{2}$. For $m$ even, the monodromy $\mathrm{M}=\mathbb{1}_{2}$ is trivial and the corresponding Mostow bundle is simply the three-torus $\mathbb{T}^{3}$, which we have already considered in Section 3.4. For $m$ odd, which we now assume, the nontrivial monodromy matrix is given by

$$
\mathrm{M}^{(1)}=\varphi_{m \pi}=-\mathbb{1}_{2} .
$$

The Euclidean $\mathbb{Z}_{2}$-solvmanimanifold $\mathbb{T}_{\mathbf{I S O}_{m}^{(1)}(2 ; \mathbb{Z})}$ is the compact space obtained as the quotient of ISO (2) with respect to the lattice given by the discrete Euclidean group

$$
\mathbf{I S O}_{m}^{(1)}(2 ; \mathbb{Z}):=\left\{\left(m \pi \alpha, \gamma_{1}, \gamma_{2}\right) \in \operatorname{ISO}(2) \mid \alpha \in \mathbb{Z}, \gamma=\left(\gamma_{1}, \gamma_{2}\right) \in \mathbb{Z}^{2}\right\}
$$

The quotient is taken by the left action of $\mathbf{I S O}_{m}^{(1)}(2 ; \mathbb{Z})$, which leads to the local coordinate identifications under the action of the generators of $\mathrm{ISO}_{m}^{(1)}(2 ; \mathbb{Z})$ given by

$$
\begin{aligned}
& \left(x, z_{1}, z_{2}\right) \longmapsto\left(x+m \pi,-z_{1},-z_{2}\right), \\
& \left(x, z_{1}, z_{2}\right) \longmapsto\left(x, z_{1}+1, z_{2}\right), \\
& \left(x, z_{1}, z_{2}\right) \longmapsto\left(x, z_{1}, z_{2}+1\right) .
\end{aligned}
$$

The relation matrix $\mathrm{A}^{(1)}=\mathrm{M}^{(1)}-\mathbb{1}_{2}=-2 \mathbb{1}_{2}$ has maximal rank $r=2$ with elementary divisors $m_{1}=m_{2}=2$, and the first homology group of $\mathbb{T}_{\mathbf{I S O}_{m}^{(1)}(2 ; \mathbb{Z})}$ can thus be presented as the $\mathbb{Z}$-module

$$
\mathrm{H}_{1}\left(\mathbb{T}_{\text {ISO }_{m}^{(1)}(2 ; \mathbb{Z})}, \mathbb{Z}\right) \simeq \mathbb{Z} \oplus\left(\mathbb{Z}_{2} \oplus \mathbb{Z}_{2}\right) .
$$

It follows that the $\mathbb{Z}_{2}$-solvmanifolds do not possess any classical T-duals. By symmetry the two possible noncommutative torus bundles corresponding to the two torsion generators $e_{z_{1}}$ and $e_{z_{2}}$ of order two are the same.

Consider the one-parameter subgroup $\mathbb{R}_{(0,1)}$ of $\mathrm{ISO}(2)$ given by

$$
\mathbb{R}_{(0,1)}=\{(0,0, \zeta) \in \operatorname{ISO}(2)\},
$$

which acts on the Euclidean group ISO(2) by right multiplication

$$
\left(x, z_{1}, z_{2}\right)(0,0, \zeta)=\left(x, z_{1}+\zeta \sin x, z_{2}+\zeta \cos x\right) .
$$

In this case $\mathbb{F}_{*}$ is the subset of $x \in \mathbb{R}$ where $\cos x=0$, so that

$$
\mathbb{F}_{*}=\frac{\pi}{2}(2 \mathbb{Z}+1) .
$$

Applying Theorem 5.1, it follows that the $C^{*}$-algebraic T-dual of the Euclidean $\mathbb{Z}_{2}$-solvmanifold is Morita equivalent to the $C(\mathbb{T})$-algebra

$$
C\left(\mathbb{T}_{\mathrm{ISO}_{m}^{(1)}(2 ; \mathbb{Z})}\right) \rtimes_{\mathrm{rt}} \mathbb{R}_{(0,1)} \sim_{\mathrm{M}} \coprod_{x \in \mathbb{R} / \mathbb{Z}} \mathbb{T}_{\theta_{m}^{(1)}(x)}^{2},
$$


where

$$
\theta_{m}^{(1)}(x)= \begin{cases}0 & \text { for } x \in \frac{1}{m}\left(\mathbb{Z}+\frac{1}{2}\right), \\ \tan (m \pi x) & \text { for } x \in \mathbb{R} \backslash \frac{1}{m}\left(\mathbb{Z}+\frac{1}{2}\right) .\end{cases}
$$

Recalling that the integer $m$ is odd here, it is easily seen that the noncommutativity parameter (5.15) is invariant under changing coset representative $x \in \mathbb{R} / \mathbb{Z}$, which is consistent with its $\mathrm{SL}(2, \mathbb{Z})$ orbit under the corresponding monodromy matrix (5.12):

$$
\theta_{m}^{(1)}(x+1)=\theta_{m}^{(1)}(x)=\mathrm{M}^{(1)}\left[\theta_{m}^{(1)}(x)\right],
$$

so that fibrewise $\mathrm{A}_{\theta_{m}^{(1)}(x+1)}=\mathrm{A}_{\theta_{m}^{(1)}(x)}$. Thus the non-trivial monodromy in the automorphism group of the $\mathbb{T}^{2}$ fibres of the twisted torus $\mathbb{T}_{\mathbf{I S O}_{m}^{(1)}(2 ; \mathbb{Z})}$ becomes a trivial identity action on its $\mathrm{T}$ dual $C^{*}$-algebra bundle. This is analogous to what we found in the parabolic case. Moreover, by Proposition 5.2 the $C^{*}$-algebra bundle (5.14) can be described as a deformation of the algebra of functions $C\left(\mathbb{T} \times \mathbb{T}^{2}\right)$ on a trivial $\mathbb{T}^{2}$-bundle over the circle via a star-product $f \star_{\theta_{m}^{(1)}} g$ given by (5.3). In marked contrast to the parabolic case, however, the algebra of functions $C\left(\mathbb{T}_{\mathbf{I S O}_{m}^{(1)}(2 ; \mathbb{Z})}\right)$ is not itself a $C\left(\mathbb{T}^{2}\right)$-algebra, and the noncommutative torus bundle cannot be identified with the group $C^{*}$-algebra of the integer Euclidean group $\mathrm{ISO}_{m}^{(1)}(2 ; \mathbb{Z})$.

\section{Euclidean $\mathbb{Z}_{4}$-solvmanifolds}

We next observe that for

$$
x_{0}=\frac{m \pi}{2}
$$

with $m$ an odd integer, the matrix $\varphi_{x_{0}}$ is again integer-valued, and so may be set equal to $\mathrm{M}$ with $\Sigma=\mathbb{1}_{2}$ :

$$
\mathrm{M}^{(2)}=\varphi_{\frac{m \pi}{2}}= \pm\left(\begin{array}{cc}
0 & 1 \\
-1 & 0
\end{array}\right) .
$$

Without loss of generality, we shall fix the positive sign by assuming that $m \in 4 \mathbb{Z}+1$ (the choice of negative sign for $m \in 4 \mathbb{Z}+3$ simply corresponds to a reflection $\left(z_{1}, z_{2}\right) \mapsto\left(-z_{1},-z_{2}\right)$ below).

The Euclidean $\mathbb{Z}_{4}$-solvmanifold $\mathbb{T}_{\mathbf{I S O}_{m}^{(2)}(2 ; \mathbb{Z})}$ is the compact space obtained as the quotient of $\mathrm{ISO}(2)$ with

$$
\mathbf{I S O}_{m}^{(2)}(2 ; \mathbb{Z}):=\left\{\left(\frac{m \pi}{2} \alpha, \gamma_{1}, \gamma_{2}\right) \in \mathbf{I S O}(2) \mid \alpha \in \mathbb{Z}, \gamma=\left(\gamma_{1}, \gamma_{2}\right) \in \mathbb{Z}^{2}\right\} .
$$

The quotient is taken by the left action of $\operatorname{ISO}_{m}^{(2)}(2 ; \mathbb{Z})$, which leads to the local coordinate identifications under the action of the generators of $\mathrm{ISO}_{m}^{(2)}(2 ; \mathbb{Z})$ given by

$$
\begin{aligned}
& \left(x, z_{1}, z_{2}\right) \longmapsto\left(x+\frac{m \pi}{2}, z_{2},-z_{1}\right), \\
& \left(x, z_{1}, z_{2}\right) \longmapsto\left(x, z_{1}+1, z_{2}\right), \\
& \left(x, z_{1}, z_{2}\right) \longmapsto\left(x, z_{1}, z_{2}+1\right) .
\end{aligned}
$$

The relation matrix $\mathrm{A}^{(2)}=\mathrm{M}^{(2)}-\mathbb{1}_{2}$ has maximal rank $r=2$ with elementary divisors $m_{1}=1$ and $m_{2}=2$, and the first homology group of $\mathbb{T}_{\mathbf{I S O}_{m}^{(2)}(2 ; \mathbb{Z})}$ can thus be presented as the $\mathbb{Z}$-module

$$
\mathrm{H}_{1}\left(\mathbb{T}_{\text {ISO }_{m}^{(2)}(2 ; \mathbb{Z})}, \mathbb{Z}\right) \simeq \mathbb{Z} \oplus \mathbb{Z}_{2},
$$

where the torsion generator of order two is $e_{z_{2}}=e_{z_{1}}$. Thus again there is no classical T-dual, as is well-known in this case (see, e.g., [28]). 
Proceeding as in the previous case, the subset $\mathbb{F}_{*} \subset \mathbb{R}$ is again given by (5.13) and we arrive at the Morita equivalence

$$
C\left(\mathbb{T}_{\mathbf{I S O}_{m}^{(2)}(2 ; \mathbb{Z})}\right) \rtimes_{\mathrm{rt}} \mathbb{R}_{(0,1)} \sim_{\mathrm{M}} \coprod_{x \in \mathbb{R} / \mathbb{Z}} \mathbb{T}_{\theta_{m}^{(2)}(x)}^{2}
$$

where

$$
\theta_{m}^{(2)}(x)= \begin{cases}0 & \text { for } x \in \frac{1}{m}(2 \mathbb{Z}+1), \\ \tan \left(\frac{m \pi}{2} x\right) & \text { for } x \in \mathbb{R} \backslash \frac{1}{m}(2 \mathbb{Z}+1) .\end{cases}
$$

The corresponding star-product $f \star_{\theta_{m}^{(2)}} g$ on $C\left(\mathbb{T} \times \mathbb{T}^{2}\right)$ is given by (5.3) and was originally written down in [28], where it was also pointed out that the noncommutative torus fibration is not isomorphic to the group $C^{*}$-algebra of $\mathrm{ISO}_{m}^{(2)}(2 ; \mathbb{Z})$.

Compared to the previous cases, the new feature here is that a change of coset representative $x \in \mathbb{R} / \mathbb{Z}$ generally has a non-trivial action on the fibers of the $C^{*}$-bundle $\coprod_{x \in \mathbb{R} / \mathbb{Z}} \mathrm{A}_{\theta_{m}^{(2)}(x)}$ according to (5.2). We check this explicitly here: Recalling that $m \in 4 \mathbb{Z}+1$ in this case, the fibre over any $x \in \frac{1}{m} \mathbb{Z}$ is preserved as then the form of (5.17) implies

$$
\theta_{m}^{(2)}(x+1)=0=\theta_{m}^{(2)}(x) \quad \text { for } \quad x \in \frac{1}{m} \mathbb{Z} .
$$

However, over any $x \in \mathbb{R} \backslash \frac{1}{m} \mathbb{Z}$ the noncommutativity parameter (5.17) changes non-trivally according to the trigonometric identity $\tan \left(x+\frac{m \pi}{2}\right)=-\cot x$, but in a way which is consistent with its $\operatorname{SL}(2, \mathbb{Z})$ orbit under the corresponding monodromy matrix (5.16):

$$
\theta_{m}^{(2)}(x+1)=-\frac{1}{\theta_{m}^{(2)}(x)}=\mathrm{M}^{(2)}\left[\theta_{m}^{(2)}(x)\right] \quad \text { for } \quad x \in \mathbb{R} \backslash \frac{1}{m} \mathbb{Z} .
$$

By Theorem 5.1, the fibre noncommutative tori are Morita equivalent, and so are isomorphic in the category $\mathscr{K} \mathscr{K}$. A physical picture of the action of the monodromy on the K-theory group of this $C^{*}$-bundle in terms of fiber D-branes is given in [28].

\section{Euclidean $\mathbb{Z}_{6}$-solvmanifolds}

Finally, we observe that for

$$
x_{0}=\frac{m \pi}{3}
$$

with $m \notin 3 \mathbb{Z}$, the matrix $\varphi_{x_{0}}$ takes four possible forms

$$
\varphi_{\frac{m \pi}{3}}=\frac{\epsilon}{2}\left(\begin{array}{cc}
\epsilon^{\prime} & \sqrt{3} \\
-\sqrt{3} & \epsilon^{\prime}
\end{array}\right)
$$

with independent signs $\epsilon, \epsilon^{\prime}= \pm 1$. It conjugates to an integer matrix via the element $\Sigma \in$ $\mathrm{SL}(2, \mathbb{R})$ given by

$$
\Sigma=\sqrt{\frac{2}{\sqrt{3}}}\left(\begin{array}{cc}
1 & \frac{1}{2} \\
0 & \frac{\sqrt{3}}{2}
\end{array}\right) .
$$

The precise form of $\varphi_{x_{0}}$ and hence of the corresponding monodromy matrix $\mathrm{M}$ depends on the congruence class of the integer $m$ in $\mathbb{Z}_{3}$ :

$$
\Sigma^{-1} \varphi_{\frac{m \pi}{3}} \Sigma= \begin{cases} \pm\left(\begin{array}{rr}
1 & 1 \\
-1 & 0
\end{array}\right), & m \in 3 \mathbb{Z}+1 \\
\pm\left(\begin{array}{rr}
0 & 1 \\
-1 & -1
\end{array}\right), & m \in 3 \mathbb{Z}+2 .\end{cases}
$$


Without loss of generality, we shall fix $m \in 6 \mathbb{Z}+2$ and hence take

$$
\mathrm{M}^{(3)}=\left(\begin{array}{cc}
0 & 1 \\
-1 & -1
\end{array}\right)
$$

in the following (with the other three possibilities obtained simply by reflection $\left(z_{1}, z_{2}\right) \mapsto$ $\left(-z_{1},-z_{2}\right)$ and interchange $\left(z_{1}, z_{2}\right) \mapsto\left(z_{2}, z_{1}\right)$ below).

The Euclidean $\mathbb{Z}_{6}$-solvmanifold $\mathbb{T}_{\mathbf{I S O}_{m}^{(3)}(2 ; \mathbb{Z})}$ is the compact space obtained as the quotient of ISO(2) with respect to the lattice given by the discrete subgroup

$$
\operatorname{ISO}_{m}^{(3)}(2 ; \mathbb{Z}):=\left\{\left(\frac{m \pi}{3} \alpha, \sqrt{\frac{2}{\sqrt{3}}} \gamma_{1}+\sqrt{\frac{1}{2 \sqrt{3}}} \gamma_{2}, \sqrt{\frac{\sqrt{3}}{2}} \gamma_{2}\right) \in \operatorname{ISO}(2) \mid \alpha \in \mathbb{Z}, \gamma=\left(\gamma_{1}, \gamma_{2}\right) \in \mathbb{Z}^{2}\right\} .
$$

The quotient is taken by the left action of $\operatorname{ISO}_{m}^{(3)}(2 ; \mathbb{Z})$, which leads to the local coordinate identifications under the action of the generators of $\operatorname{ISO}_{m}^{(3)}(2 ; \mathbb{Z})$ given by

$$
\begin{aligned}
& \left(x, z_{1}, z_{2}\right) \longmapsto\left(x+\frac{m \pi}{3}, \frac{1}{2}\left(\sqrt{3} z_{2}-z_{1}\right),-\frac{1}{2}\left(\sqrt{3} z_{1}+z_{2}\right)\right), \\
& \left(x, z_{1}, z_{2}\right) \longmapsto\left(x, z_{1}+\sqrt{\frac{2}{\sqrt{3}}}, z_{2}\right), \\
& \left(x, z_{1}, z_{2}\right) \longmapsto\left(x, z_{1}+\sqrt{\frac{1}{2 \sqrt{3}}}, z_{2}+\sqrt{\frac{\sqrt{3}}{2}}\right) .
\end{aligned}
$$

The relation matrix $\mathrm{A}^{(3)}=\mathrm{M}^{(3)}-\mathbb{1}_{2}$ has maximal rank $r=2$ with elementary divisors $m_{1}=1$ and $m_{2}=3$, and the first homology group can thus be presented as the $\mathbb{Z}$-module

$$
\mathrm{H}_{1}\left(\mathbb{T}_{\text {ISO }_{m}^{(3)}(2 ; \mathbb{Z})}, \mathbb{Z}\right) \simeq \mathbb{Z} \oplus \mathbb{Z}_{3},
$$

where the torsion generator of order three is $e_{z_{2}}=e_{z_{1}}$. Here too there is no classical T-dual.

Applying Theorem 5.1 in this case, with the non-trivial period matrix $\Sigma$ from (5.18) and the subset $\mathbb{F}_{*} \subset \mathbb{R}$ again given by (5.13), gives the Morita equivalence

$$
C\left(\mathbb{T}_{\mathrm{ISO}_{m}^{(3)}(2 ; \mathbb{Z})}\right) \rtimes_{\mathrm{rt}} \mathbb{R}_{(0,1)} \sim_{\mathrm{M}} \coprod_{x \in \mathbb{R} / \mathbb{Z}} \mathbb{T}^{2} \theta_{m}^{(3)}(x),
$$

where

$$
\theta_{m}^{(3)}(x)= \begin{cases}0 & \text { for } x \in \frac{1}{m}\left(3 \mathbb{Z}+\frac{3}{2}\right), \\ -\frac{1}{2}+\frac{\sqrt{3}}{2} \tan \left(\frac{m \pi}{3} x\right) & \text { for } x \in \mathbb{R} \backslash \frac{1}{m}\left(3 \mathbb{Z}+\frac{3}{2}\right) .\end{cases}
$$

As in the previous case, changing coset representative $x \in \mathbb{R} / \mathbb{Z}$ generally has a non-trivial action on the fibers of the $C^{*}$-bundle over $\mathbb{T}$ : Recalling that $m \in 6 \mathbb{Z}+2$ here, the fiber over any $x \in \frac{1}{m}\left(3 \mathbb{Z}+\frac{3}{2}\right)$ is preserved by the form of $(5.20)$ :

$$
\theta_{m}^{(3)}(x+1)=0=\theta_{m}^{(3)}(x) \quad \text { for } \quad x \in \frac{1}{m}\left(3 \mathbb{Z}+\frac{3}{2}\right) .
$$

On the other hand, over any $x \in \mathbb{R} \backslash \frac{1}{m}\left(3 \mathbb{Z}+\frac{3}{2}\right)$ the noncommutativity parameter (5.20) changes according to the trigonometric identity

$$
\tan \left(x+\frac{m \pi}{3}\right)=\frac{\tan x-\sqrt{3}}{1+\sqrt{3} \tan x},
$$

but consistently with its $\mathrm{SL}(2, \mathbb{Z})$ orbit under the corresponding monodromy matrix (5.19):

$$
\theta_{m}^{(3)}(x+1)=-\frac{1}{\theta_{m}^{(3)}(x)+1}=\mathrm{M}^{(3)}\left[\theta_{m}^{(3)}(x)\right] \quad \text { for } \quad x \in \mathbb{R} \backslash \frac{1}{m}\left(3 \mathbb{Z}+\frac{3}{2}\right) .
$$

By Theorem 5.1 the corresponding fibre noncommutative tori are Morita equivalent, and so isomorphic in the category $\mathscr{K} \mathscr{K}$. 


\subsection{Hyperbolic torus bundles}

Our last application of Theorem 5.1 is to the final class of three-dimensional solvmanifolds, which are hyperbolic analogues of the Euclidean solvmanifolds based on the Poincaré group, and have also not been previously studied in the present context.

The Poincaré group ISO $(1,1)$ in two dimensions is the three-dimensional almost abelian solvable Lie group which can be presented as

$$
\operatorname{ISO}(1,1)=\mathbb{R}^{2} \rtimes_{\varphi} \mathbb{R} \quad \text { with } \quad \varphi_{x}=\left(\begin{array}{cc}
\cosh x & \sinh x \\
\sinh x & \cosh x
\end{array}\right) .
$$

Since $\left|\operatorname{Tr} \varphi_{x}\right|>2$ for all $x \in \mathbb{R}^{\times}$, the hyperbolic rotation $\varphi_{x}$ parameterizes a hyperbolic conjugacy class of $\operatorname{SL}(2, \mathbb{R})$. Again we denote coordinates on the group manifold of $\mathbf{N}=\mathbb{R}^{2}$ by $z=\left(z_{1}, z_{2}\right)$, so that the group multiplication on $\mathrm{ISO}(1,1)$ is given by

$$
\left(x, z_{1}, z_{2}\right)\left(x^{\prime}, z_{1}^{\prime}, z_{2}^{\prime}\right)=\left(x+x^{\prime}, z_{1}+z_{1}^{\prime} \cosh x+z_{2}^{\prime} \sinh x, z_{2}+z_{1}^{\prime} \sinh x+z_{2}^{\prime} \cosh x\right)
$$

and the inverse of a group element is

$$
\left(x, z_{1}, z_{2}\right)^{-1}=\left(-x,-z_{1} \cosh x+z_{2} \sinh x, z_{1} \sinh x-z_{2} \cosh x\right) .
$$

Clearly there is no $x_{0} \in \mathbb{R}^{\times}$for which $\varphi_{x_{0}}$ is an integer matrix in this case. However, there is an infinite family of discrete points

$$
x_{0}=\log \left(\frac{m}{2} \pm \sqrt{\left(\frac{m}{2}\right)^{2}-1}\right)
$$

labelled by an integer $m>2$ with $2 \cosh x_{0}=m$, at which the matrix $\varphi_{x_{0}}$ takes the form

$$
\varphi_{x_{0}}=\frac{1}{2}\left(\begin{array}{cc}
m & \pm \sqrt{m^{2}-4} \\
\pm \sqrt{m^{2}-4} & m
\end{array}\right) .
$$

This matrix has eigenvalues $\left(\lambda, \lambda^{-1}\right)$ where

$$
\lambda^{ \pm 1}=\frac{1}{2}\left(m \pm \sqrt{m^{2}-4}\right)=\mathrm{e}^{x_{0}},
$$

and it conjugates to the integer matrix

$$
\mathrm{M}_{m}=\Sigma^{-1} \varphi_{x_{0}} \Sigma=\left(\begin{array}{cc}
m & 1 \\
-1 & 0
\end{array}\right)
$$

by the element $\Sigma \in \mathrm{SL}(2, \mathbb{R})$ given by

$$
\Sigma=\left(\begin{array}{cc}
\frac{\lambda^{ \pm 1}}{2\left(\lambda-\lambda^{-1}\right)}-\lambda^{\mp 1} & \frac{\lambda}{2\left(\lambda^{2}-1\right)}-1 \\
\frac{\lambda^{ \pm 1}}{2\left(\lambda-\lambda^{-1}\right)}+\lambda^{\mp 1} & \frac{\lambda}{2\left(\lambda^{2}-1\right)}+1
\end{array}\right) .
$$

For definiteness, we choose the positive square root in (5.21) (with the choice of negative square root obtained from the interchange $\left(z_{1}, z_{2}\right) \mapsto\left(z_{2}, z_{1}\right)$ below).

The Poincaré solvmanifold $\mathbb{T}_{\mathbf{I S O}_{m}(1,1 ; \mathbb{Z})}$ is the compact space obtained as the quotient of ISO $(1,1)$ by the lattice

$$
\operatorname{ISO}_{m}(1,1 ; \mathbb{Z}):=\left\{\left(x_{0} \alpha, \Sigma \cdot \gamma\right) \in \operatorname{ISO}(1,1) \mid \alpha \in \mathbb{Z}, \gamma=\left(\gamma_{1}, \gamma_{2}\right) \in \mathbb{Z}^{2}\right\} .
$$


The quotient by the left action of this lattice leads to the local coordinate identifications under the action of the generators given by

$$
\begin{aligned}
&\left(x, z_{1}, z_{2}\right) \longmapsto\left(x+\cosh ^{-1}\left(\frac{m}{2}\right), \frac{1}{2}\left(m z_{1}+\sqrt{m^{2}-4} z_{2}\right), \frac{1}{2}\left(\sqrt{m^{2}-4} z_{1}+m z_{2}\right)\right), \\
&\left(x, z_{1}, z_{2}\right) \longmapsto\left(x, z_{1}+\frac{\lambda^{2}}{2\left(\lambda^{2}-1\right)}-\lambda^{-1}, z_{2}+\frac{\lambda^{2}}{2\left(\lambda^{2}-1\right)}+\lambda^{-1}\right), \\
&\left(x, z_{1}, z_{2}\right) \longmapsto\left(x, z_{1}+\frac{\lambda}{2\left(\lambda^{2}-1\right)}-1, z_{2}+\frac{\lambda}{2\left(\lambda^{2}-1\right)}+1\right) .
\end{aligned}
$$

The relation matrix $\mathrm{A}_{m}=\mathrm{M}_{m}-\mathbb{1}_{2}$ has maximal rank $r=2$ with elementary divisors $m_{1}=1$ and $m_{2}=m-2$, so the first homology group of the Poincaré solvmanifold may be presented as the $\mathbb{Z}$-module

$$
\mathrm{H}_{1}\left(\mathbb{T}_{\mathrm{ISO}_{m}(1,1 ; \mathbb{Z})}, \mathbb{Z}\right) \simeq \mathbb{Z} \oplus \mathbb{Z}_{m-2}
$$

with torsion generator $e_{z_{2}}=-e_{z_{1}}$ of order $m-2$.

Consider the one-parameter subgroup

$$
\mathbb{R}_{(0,1)}=\{(0,0, \zeta) \in \operatorname{ISO}(1,1)\}
$$

acting on the Poincaré group ISO $(1,1)$ by right multiplication

$$
\left(x, z_{1}, z_{2}\right)(0,0, \zeta)=\left(x, z_{1}+\zeta \sinh x, z_{2}+\zeta \cosh x\right) .
$$

In this case the set $\mathbb{F}_{*}$ consists of a distinguished point $x_{*} \in \mathbb{R}$ on the base of the $C^{*}$-algebra bundle given by

$$
x_{*}=\tanh ^{-1}\left(\frac{2\left(\lambda^{2}-1\right)-\lambda^{3}}{2\left(\lambda^{2}-1\right)+\lambda^{3}}\right) .
$$

Applying Theorem 5.1, with the periods (5.21) and (5.23), then identifies the Morita equivalence

$$
C\left(\mathbb{T}_{\mathbf{I S O}_{m}(1,1 ; \mathbb{Z})}\right) \rtimes_{\mathrm{rt}} \mathbb{R}_{(0,1)} \sim_{\mathrm{M}} \coprod_{x \in \mathbb{R} / \mathbb{Z}} \mathbb{T}_{\theta_{m}(x)}^{2}
$$

of $C(\mathbb{T})$-algebras, where

$$
\theta_{m}\left(x_{*}\right)=0
$$

and

$$
\theta_{m}(x)=\frac{2 \lambda\left(\lambda^{2}-1\right)-\lambda^{2}+\lambda\left(2\left(\lambda^{2}-1\right)+\lambda\right) \tanh \left(\cosh ^{-1}\left(\frac{m}{2}\right) x\right)}{\lambda^{3}-2\left(\lambda^{2}-1\right)-\left(\lambda^{3}+2\left(\lambda^{2}-1\right)\right) \tanh \left(\cosh ^{-1}\left(\frac{m}{2}\right) x\right)}
$$

for $x \neq x_{*}$.

This noncommutative torus bundle, along with its topological T-duality with $C\left(\mathbb{T}_{\operatorname{ISO}_{m}(1,1 ; \mathbb{Z})}\right)$, has formally the same properties as the $C(\mathbb{T})$-algebras described in Section 5.3, so we refrain from repeating the details here. We only mention the fibre monodromy behaviour anticipated from (5.2): Using the hyperbolic identity

$$
\tanh \left(x+\cosh ^{-1}\left(\frac{m}{2}\right)\right)=\frac{m \tanh x+\sqrt{m^{2}-4}}{m+\sqrt{m^{2}-4} \tanh x},
$$

we find

$$
\theta_{m}\left(x_{*}+1\right)=0=\theta_{m}\left(x_{*}\right)
$$


and we see explicitly here that the noncommutativity parameter (5.24) changes consistently with its $\mathrm{SL}(2, \mathbb{Z})$ orbit under the monodromy matrix (5.22):

$$
\theta_{m}(x+1)=-\frac{1}{\theta_{m}(x)}-m=\mathrm{M}_{m}\left[\theta_{m}(x)\right] \quad \text { for } \quad x \neq x_{*} .
$$

Thus the fiber noncommutative tori are Morita equivalent by Theorem 5.1, and so are isomorphic $C^{*}$-algebras in the category $\mathscr{K} \mathscr{K}$.

\section{Acknowledgments}

We thank Ryszard Nest and Erik Plauschinn for helpful discussions. We thank the anonymous referees for their detailed suggestions. This research was supported by funds from Università del Piemonte Orientale (UPO). P.A. acknowledges partial support from INFN, CSN4, and Iniziativa Specifica GSS. P.A. is affiliated to INdAM-GNFM. R.J.S. acknowledges a Visiting Professorship through UPO Internationalization Funds. R.J.S. also acknowledges the Arnold-Regge Centre for the visit, and INFN. The work of R.J.S. was supported in part by the Consolidated Grant ST/P000363/1 from the UK Science and Technology Facilities Council.

\section{References}

[1] Andriot D., New supersymmetric vacua on solvmanifolds, J. High Energy Phys. 2016 (2016), no. 2, 112, 43 pages, arXiv:1507.00014.

[2] Bock C., On low-dimensional solvmanifolds, Asian J. Math. 20 (2016), 199-262, arXiv:0903.2926.

[3] Bouwknegt P., Evslin J., Mathai V., T-duality: topology change from H-flux, Comm. Math. Phys. 249 (2004), 383-415, arXiv:hep-th/0306062.

[4] Bouwknegt P., Pande A.S., Topological T-duality and T-folds, Adv. Theor. Math. Phys. 13 (2009), 15191539, arXiv:0810.4374.

[5] Brace D., Morariu B., Zumino B., Dualities of the Matrix model from T-duality of the type II string, Nuclear Phys. B 545 (1999), 192-216, arXiv:hep-th/9810099.

[6] Brodzki J., Mathai V., Rosenberg J., Szabo R.J., D-branes, RR-fields and duality on noncommutative manifolds, Comm. Math. Phys. 277 (2008), 643-706, arXiv:hep-th/0607020.

[7] Brodzki J., Mathai V., Rosenberg J., Szabo R.J., Non-commutative correspondences, duality and D-branes in bivariant K-theory, Adv. Theor. Math. Phys. 13 (2009), 497-552, arXiv:0708.2648.

[8] Bunke U., Rumpf P., Schick T., The topology of T-duality for $T^{n}$-bundles, Rev. Math. Phys. 18 (2006), 1103-1154, arXiv:math.GT/0501487.

[9] Bunke U., Schick T., On the topology of T-duality, Rev. Math. Phys. 17 (2005), 77-112, arXiv:math.GT/0405132.

[10] Console S., Macrì M., Lattices, cohomology and models of 6-dimensional almost abelian solvmanifolds, Rend. Semin. Mat. Univ. Politec. Torino 74 (2016), 95-119, arXiv:1206.5977.

[11] Corwin L.J., Greenleaf F.P., Representations of nilpotent Lie groups and their applications. Part I. Basic theory and examples, Cambridge Studies in Advanced Mathematics, Vol. 18, Cambridge University Press, Cambridge, 1990.

[12] Cowen C.C., MacCluer B.D., Linear fractional maps of the ball and their composition operators, Acta Sci. Math. (Szeged) 66 (2000), 351-376.

[13] Dabholkar A., Hull C., Duality twists, orbifolds, and fluxes, J. High Energy Phys. 2003 (2003), no. 9, 054, 25 pages, arXiv:hep-th/0210209.

[14] Echterhoff S., Crossed products and the Mackey-Rieffel-Green machine, Oberwolfach Sem. 47 (2017), 5-79, arXiv:1006.4975.

[15] Echterhoff S., Nest R., Oyono-Oyono H., Principal non-commutative torus bundles, Proc. Lond. Math. Soc. 99 (2009), 1-31, arXiv:0810.0811. 
[16] Ellwood I., Hashimoto A., Effective descriptions of branes on non-geometric tori, J. High Energy Phys. 2006 (2006), no. 12, 025, 22 pages, arXiv:hep-th/0607135.

[17] Fack T., Skandalis G., Connes' analogue of the Thom isomorphism for the Kasparov groups, Invent. Math. 64 (1981), 7-14.

[18] Fröhlich J., Gawȩdzki K., Conformal field theory and geometry of strings, in Mathematical Quantum Theory. I. Field Theory and Many-Body Theory (Vancouver, BC, 1993), CRM Proc. Lecture Notes, Vol. 7, Amer. Math. Soc., Providence, RI, 1994, 57-97, arXiv:hep-th/9310187.

[19] Grange P., Schäfer-Nameki S., T-duality with $H$-flux: non-commutativity, $T$-folds and $G \times G$ structure, Nuclear Phys. B $\mathbf{7 7 0}$ (2007), 123-144, arXiv:hep-th/0609084.

[20] Green P., The structure of imprimitivity algebras, J. Funct. Anal. 36 (1980), 88-104.

[21] Hannabuss K.C., Mathai V., Noncommutative principal torus bundles via parametrised strict deformation quantization, in Superstrings, Geometry, Topology, and $C^{*}$-Algebras,Proc. Sympos. Pure Math., Vol. 81, Amer. Math. Soc., Providence, RI, 2010, 133-147, arXiv:0911.1886.

[22] Hannabuss K.C., Mathai V., Parametrized strict deformation quantization of $C^{*}$-bundles and Hilbert $C^{*}$ modules, J. Aust. Math. Soc. 90 (2011), 25-38, arXiv:1007.4696.

[23] Havas G., Majewski B.S., Integer matrix diagonalization, J. Symbolic Comput. 24 (1997), 399-408.

[24] Hori K., D-branes, T-duality, and index theory, Adv. Theor. Math. Phys. 3 (1999), 281-342, arXiv:hepth/9902102.

[25] Hull C.M., A geometry for non-geometric string backgrounds, J. High Energy Phys. 2005 (2005), no. 10, 065, 30 pages, arXiv:hep-th/0406102.

[26] Hull C.M., Reid-Edwards R.A., Flux compactifications of string theory on twisted tori, Fortschr. Phys. 57 (2009), 862-894, arXiv:hep-th/0503114.

[27] Hull C.M., Reid-Edwards R.A., Non-geometric backgrounds, doubled geometry and generalised T-duality, J. High Energy Phys. 2009 (2009), no. 9, 014, 79 pages, arXiv:0902.4032.

[28] Hull C.M., Szabo R.J., Noncommutative gauge theories on D-branes in non-geometric backgrounds, J. High Energy Phys. 2019 (2019), no. 9, 051, 40 pages, arXiv:1903.04947.

[29] Landi G., Lizzi F., Szabo R.J., String geometry and the noncommutative torus, Comm. Math. Phys. 206 (1999), 603-637, arXiv:hep-th/9806099.

[30] Lizzi F., Szabo R.J., Duality symmetries and noncommutative geometry of string spacetimes, Comm. Math. Phys. 197 (1998), 667-712, arXiv:hep-th/9707202.

[31] Lowe D.A., Nastase H., Ramgoolam S., Massive IIA string theory and matrix theory compactification, Nuclear Phys. B 6667 (2003), 55-89, arXiv:hep-th/0303173.

[32] Mathai V., Rosenberg J., T-duality for torus bundles with $H$-fluxes via noncommutative topology, Comm. Math. Phys. 253 (2005), 705-721, arXiv:hep-th/0401168.

[33] Mathai V., Rosenberg J., T-duality for torus bundles with $H$-fluxes via noncommutative topology. II. The high-dimensional case and the T-duality group, Adv. Theor. Math. Phys. 10 (2006), 123-158, arXiv:hepth/0508084.

[34] Milnor J., Curvatures of left invariant metrics on Lie groups, Adv. Math. 21 (1976), 293-329.

[35] Mostow G.D., Factor spaces of solvable groups, Ann. of Math. 60 (1954), 1-27.

[36] Nikolaus T., Waldorf K., Higher geometry for non-geometric T-duals, Comm. Math. Phys. 374 (2020), 317-366, arXiv:1804.00677.

[37] Phillips N.C., Every simple higher-dimensional noncommutative torus is an AT algebra, arXiv:math.OA/0609783.

[38] Pioline B., Schwarz A., Morita equivalence and T-duality (or B versus $\Theta$ ), J. High Energy Phys. 1999 (1999), no. 8, 021, 16 pages, arXiv:hep-th/9908019.

[39] Raeburn I., Rosenberg J., Crossed products of continuous-trace $C^{*}$-algebras by smooth actions, Trans. Amer. Math. Soc. 305 (1988), 1-45.

[40] Raghunathan M.S., Discrete subgroups of Lie groups, Ergebnisse der Mathematik und ihrer Grenzgebiete, Vol. 68, Springer-Verlag, New York - Heidelberg, 1972.

[41] Rieffel M.A., Induced representations of $C^{*}$-algebras, Adv. Math. 13 (1974), 176-257.

[42] Rieffel M.A., Strong Morita equivalence of certain transformation group $C^{*}$-algebras, Math. Ann. 222 (1976), $7-22$. 
[43] Rieffel M.A., $C^{*}$-algebras associated with irrational rotations, Pacific J. Math. 93 (1981), 415-429.

[44] Rieffel M.A., The cancellation theorem for projective modules over irrational rotation $C^{*}$-algebras, Proc. London Math. Soc. 47 (1983), 285-302.

[45] Rieffel M.A., Noncommutative tori - a case study of noncommutative differentiable manifolds, in Geometric and Topological Invariants of Elliptic Operators (Brunswick, ME, 1988), Contemp. Math., Vol. 105, Amer. Math. Soc., Providence, RI, 1990, 191-211.

[46] Rieffel M.A., Schwarz A., Morita equivalence of multidimensional noncommutative tori, Internat. J. Math. 10 (1999), 289-299, arXiv:math.QA/9803057.

[47] Schwarz A., Morita equivalence and duality, Nuclear Phys. B 534 (1998), 720-738, arXiv:hep-th/9805034.

[48] Seiberg N., Witten E., String theory and noncommutative geometry, J. High Energy Phys. 1999 (1999), no. 9, 032, 93 pages, arXiv:hep-th/9908142.

[49] Steenrod N., The Topology of Fibre Bundles, Princeton Mathematical Series, Vol. 14, Princeton University Press, Princeton, N.J., 1951.

[50] Tralle A., Oprea J., Symplectic manifolds with no Kähler structure, Lecture Notes in Mathematics, Vol. 1661, Springer-Verlag, Berlin, 1997.

[51] Williams D.P., The structure of crossed products by smooth actions, J. Austral. Math. Soc. Ser. A 47 (1989), 226-235.

[52] Williams D.P., Crossed products of $C^{*}$-algebras, Mathematical Surveys and Monographs, Vol. 134, Amer. Math. Soc., Providence, RI, 2007. 